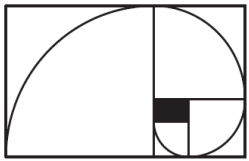

\title{
From random walk trajectories to random interlacements
}

\author{
Jiří Černý \\ Augusto Quadros Teixeira
}

\begin{abstract}
We review and comment recent research on random interlacements model introduced by A.-S. Sznitman in the paper 'Vacant set of random interlacements and percolation' (Ann. of Math. 171 (2010)). A particular emphasis is put on motivating the definition of the model via natural questions concerning geometrical/percolative properties of random walk trajectories on finite graphs, as well as on presenting some important techniques used in random interlacements' literature in the most accessible way. This text is an expanded version of the lecture notes for the mini-course given at the XV Brazilian School of Probability in 2011.
\end{abstract}

2010 Mathematics Subject Classification: 60G50, 60K35, 82C41, $05 \mathrm{C} 80$. 



\section{Acknowledgments}

This survey article is based on the lecture notes for the mini-course on random interlacements offered at the XV Brazilian School of Probability, from 31st July to 6th August 2011. We would like to thank the organizers and sponsors of the conference for providing such opportunity, especially Claudio Landim for the invitation. We are grateful to David Windisch for simplifying several of the arguments in these notes and to A. Drewitz, R. Misturini, B. Gois and G. Montes de Oca for reviewing earlier versions the text. We would also like to thank the anonymous referees for many useful comments and corrections. 



\section{Contents}

\begin{tabular}{lll}
\hline & Introduction & 7
\end{tabular}

2 Random walk on the torus 13

2.1 Notation . . . . . . . . . . . . . . . . . . . . . . . 13

2.2 Local entrance point . . . . . . . . . . . . . . . . . . 15

2.3 Local measure . . . . . . . . . . . . . . . . . . . . . . . . . 19

2.4 Local picture as Poisson point process . . . . . . . . . 22

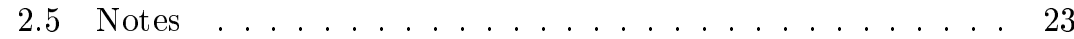

2.5 .1 Disconnection of a discrete cylinder. . . . . . . . . 24

$\begin{array}{|lr|}3 & \text { Definition of random interlacements }\end{array}$

3.1 Construction of random interlacements . . . . . . . . . . 27

3.2 Notes . . . . . . . . . . . . . . . . . 34

\begin{tabular}{|ll|}
\hline & Properties of random interlacements
\end{tabular}

4.1 Basic properties . . . . . . . . . . . . . . 35

4.2 Translation invariance and ergodicity . . . . . . . . . . . 37

4.3 Comparison with Bernoulli percolation . . . . . . . . . . . 40

4.4 Notes $\ldots \ldots \ldots \ldots \ldots \ldots$. . . . . . . . . . . . . . 44

5 Renormalization 45

5.1 Renormalization scheme . . . . . . . . . . . . . . . . 45

5.2 Notes $\ldots \ldots \ldots \ldots \ldots \ldots \ldots$

$6 \quad$ Interlacement set 51

6.1 Main properties of random interlacements . . . . . . . . . 51

6.2 Notes $\ldots \ldots \ldots \ldots \ldots \ldots \ldots \ldots$

\begin{tabular}{|lll}
7 & Locally tree-like graphs & 60
\end{tabular}

7.1 Random interlacements on trees. . . . . . . . . . . . . . 60

7.2 Random walk on tree-like graphs . . . . . . . . . . . 63

7.2.1 Very short introduction to random graphs . . . . . . 65

7.2 .2 Distribution of the vacant set . . . . . . . . . 66 
7.2.3 Random graphs with a given degree sequence. . . . . 68 7.2 .4 The degree sequence of the vacant graph . . . . . . 68

7.3 Notes $\ldots \ldots \ldots \ldots \ldots \ldots$

$\begin{array}{ll}\text { Index } & 71\end{array}$

\begin{tabular}{ll}
\hline Bibliography & 73
\end{tabular} 


\section{Chapter 1}

\section{Introduction}

These notes are based on the mini-course offered at the 'XV Brazilian School of Probability' in Mambucaba in August 2011. The lectures tried to introduce the audience to random interlacements, a 'dependentpercolation' model recently introduced by A.-S. Sznitman in [47. The emphasis was put on motivating the definition of this model via some natural questions about random walks on finite graphs, explaining the difficulties that appear when studying the model, and presenting some of the techniques used to analyze random interlacements. We tried to present these techniques in the simplest possible way, sometimes at expense of generality.

Let us start setting the stage for this review by introducing one of the problems which motivated the definition of random interlacements. To this end, fix a finite graph $G=(V, \mathcal{E})$ with a vertex set $V$ and an edge set $\mathcal{E}$, and denote by $\left(X_{n}\right)_{n \geq 0}$ a simple random walk on this graph, that is the Markovian movement of a particle on $G$ prescribed as follows: It starts at a given (possibly random) vertex $x \in G, X_{0}=x$, and given the position at time $k \geq 0$, say $X_{k}=y$, its position $X_{k+1}$ at time $k+1$ is uniformly chosen among all neighbors of $y$ in $G$.

Random walks on finite and infinite graphs, in particular on $\mathbb{Z}^{d}$, has been subject of intense research for a long time. Currently, there is a great deal of studying material on this subject, see for instance the monographs [27, 28, 29, 42, 58. Nevertheless, there are still many interesting questions and vast areas of research which are still to be further explored.

The question that will be of our principal interest was originally asked by M.J. Hilhorst, who proposed the random walk as a toy model for corrosion of materials. For sake of concreteness, take the graph $G$ to be the $d$-dimensional discrete torus $\mathbb{T}_{N}^{d}=(\mathbb{Z} / N \mathbb{Z})^{d}$ which for $d=3$ can be regarded as a piece of crystalline solid. The torus is made into a graph by adding edges between two points at Euclidean distance one from each 
other. Consider now a simple random walk $\left(X_{n}\right)_{n \geq 0}$, and imagine that this random walk represents a corrosive particle wandering erratically through the crystal, while it marks all visited vertices as 'corroded'. (The particle can revisit corroded vertices, so its dynamics is Markovian, i.e. it is not influenced by its past.)

If the time that the particle runs is short, then one intuitively expects that only a small part of the torus will be corroded, the crystal will be 'intact'. On the other hand, when the running time is large, many sites will be corroded and the connected components of non-corroded sites will be small, the crystal will be destroyed by the corrosion, see Figure 1.1 for the simulations. The question is how long should the particle run to destroy the crystal and how this destruction proceeds.
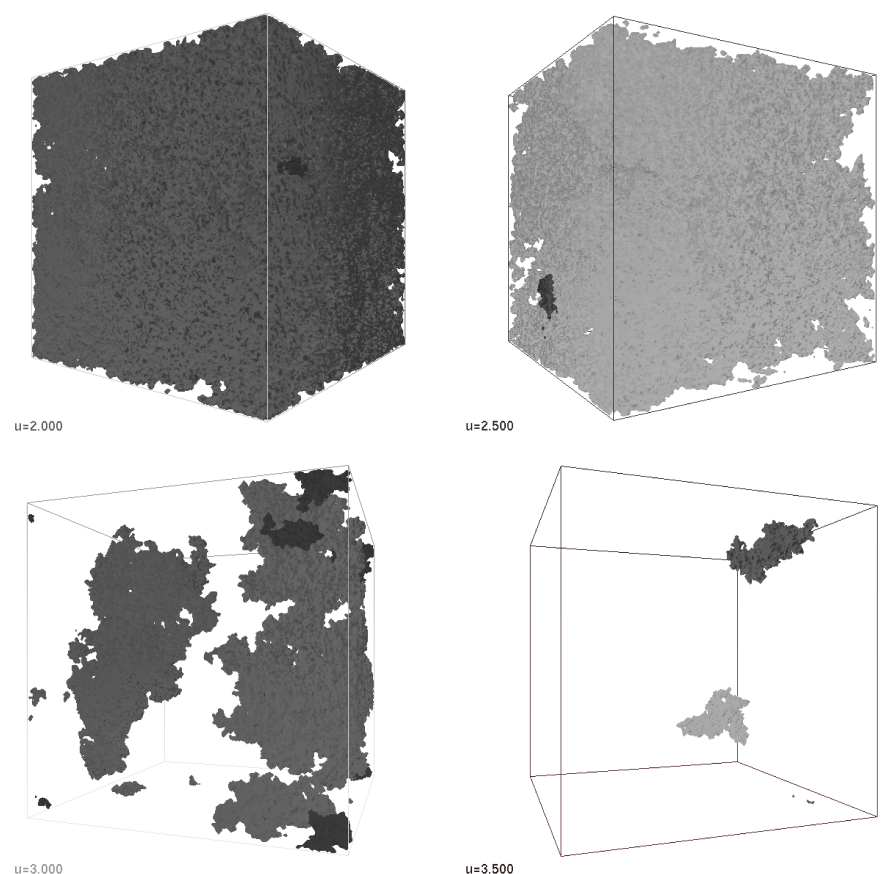

Figure 1.1: A computer simulation by David Windisch of the largest component (light gray) and second largest component (dark gray) of the vacant set left by a random walk on $(\mathbb{Z} / N \mathbb{Z})^{3}$ after $\left[u N^{3}\right]$ steps, for $N=200$. The pictures correspond consecutively to $u$ being $2.0,2.5,3.0$, and 3.5. According to recent simulation, the threshold of the phase transition satisfies $u_{c}\left(\mathbb{T}^{3}\right)=2.95 \pm 0.1$.

Remark that throughout these notes, we will not be interested in the 
instant when all sites become corroded, that is in the cover time of the graph by the simple random walk. Note however that random interlacements can also be useful when studying this problem, see the recent papers [3, 4] of D. Belius.

In a more mathematical language, let us define the vacant set left by the random walk on the torus up to time $n$

$$
\mathcal{V}_{N}(n)=\mathbb{T}_{N}^{d} \backslash\left\{X_{0}, X_{1}, \ldots, X_{n}\right\} .
$$

$\mathcal{V}_{N}(n)$ is the set of non-visited sites at time $n$ (or simply the set noncorroded sites at this time). We are interested in connectivity properties of the vacant set, in particular in the size of its largest connected component.

We will see later that the right way to scale $n$ with $N$ is $n=u N^{d}$ for $u \geq 0$ fixed. In this scaling the density of the vacant set is asymptotically constant and non-trivial, that is is for every $x \in \mathbb{T}_{N}^{d}$,

$$
\lim _{N \rightarrow \infty} \operatorname{Prob}\left[x \in \mathcal{V}_{N}\left(u N^{d}\right)\right]=c(u, d) \in(0,1) .
$$

This statement suggest to view our problem from a slightly different perspective: as a specific site percolation model on the torus with density (roughly) $c(u, d)$, but with spatial correlations. These correlations decay rather slowly with the distance, which makes the understanding of the model delicate.

At this point it is useful to recall some properties of the usual Bernoulli site percolation on the torus $\mathbb{T}_{N}^{d}, d \geq 2$, that is of the model where the sites are declared open (non-corroded) and closed (corroded) independently with respective probabilities $p$ and $1-p$. This model exhibits a phase transition at a critical value $p_{c} \in(0,1)$. More precisely, when $p<p_{c}$, the largest connected open cluster $\mathcal{C}_{\max }^{\star}(p)$ is small with high probability,

$$
p<p_{c} \quad \Longrightarrow \quad \lim _{N \rightarrow \infty} \operatorname{Prob}\left[\left|\mathcal{C}_{\max }^{\star}(p)\right|=O\left(\log ^{\lambda} N\right)\right]=1,
$$

and when $p>p_{c}$, the largest connected open cluster is comparable with the whole graph (it is then called giant),

$$
p>p_{c} \quad \Longrightarrow \quad \lim _{N \rightarrow \infty} \operatorname{Prob}\left[\left|\mathcal{C}_{\max }^{\star}(p)\right| \sim N^{d}\right]=1 .
$$

Much more is known about this phase transition, at least when $d$ is large [9, 10. A similar phase transition occurs on other (sequences of) finite graphs, in particular on large complete graph, where it was discovered (for the edge percolation) in the celebrated paper of Erdôs and Rényi [19].

Coming back to our random walk problem, we may now refine our questions: Does a similar phase transition occur there? Is there a critical value $u_{c}=u_{c}\left(\mathbb{T}^{d}\right)$ such that, using $\mathcal{C}_{\max }(u, N)$ to denote the largest connected 
component of the vacant set on $\mathbb{T}_{N}^{d}$ at time $u N^{d}$,

$$
\begin{aligned}
& u<u_{c} \quad \Longrightarrow \quad \lim _{N \rightarrow \infty} \operatorname{Prob}\left[\left|\mathcal{C}_{\max }(u, N)\right| \sim N^{d}\right]=1, \\
& u>u_{c} \quad \Longrightarrow \quad \lim _{N \rightarrow \infty} \operatorname{Prob}\left[\left|\mathcal{C}_{\max }(u, N)\right|=o\left(N^{d}\right)\right]=1 ?
\end{aligned}
$$

At the time of writing of these notes, we have only partial answers to to this question (see Chapter 2 below). It is however believed that this phase transition occurs for the torus in any dimension $d \geq 3$. This belief is supported by simulations, cf. Figure 1.1 .

Remark 1.1. It is straightforward to see that such phase transition does not occur for $d \in\{1,2\}$. When $d=1$, the vacant set at time $n$ is a segment of length roughly $\left(N-\left(n^{1 / 2} \xi\right)\right) \vee 0$, where $\xi$ is a random variable distributed as the size of the range of a Brownian motion at time 1 . Therefore, the scaling $n=u N^{d}=u N$ in 1.2 is not interesting. More importantly, it follows from this fact that no sharp phase transition occurs even on the 'corrected' scaling, $n=u N^{2}$. The situation in $d=2$ is more complicated, but the fragmentation is qualitatively different from the case $d \geq 3$.

The phase transition for the Bernoulli percolation on the torus, (1.3), (1.4), can be deduced straightforwardly from the properties of its 'infinite volume limit', that is the Bernoulli percolation on $\mathbb{Z}^{d}$ which is very well understood (see e.g. the monographs [20, 8]). It is thus legitimate to ask whether there is an infinite volume percolation which is a local limit of the vacant set. This question is not only of theoretical interest. There are many problems that are easier to solve in the infinite volume situation. As we will see, the existence of the phase transition is one of them.

One of the main goals of these notes is to construct explicitly this infinite volume limit, which is called random interlacements, and study its properties. We will not go into details how these properties can be then used to control the finite volume problem, even if not surprisingly, the recent progress in understanding random interlacements has been useful to analyze the original questions concerning the vacant set on the torus, see [53].

\section{Organization of these notes}

In the first chapters of these notes we restrict our analysis to $d$-dimensional torus and $\mathbb{Z}^{d}$, with $d \geq 3$, cf. Remark 1.1 .

In Chapter 2 we study the random walk on the torus, aiming on motivating the construction of random interlacements. We will define what we call the 'local-picture' left by the random walk on $\mathbb{T}_{N}^{d}$. To be more precise, suppose that $N$ is large and that we are only interested in what happens in a small fixed box $A \subset \mathbb{T}_{N}^{d}$. It is clear that as the number of steps $n$ 
of the walk grows, the random walk will visit $A$ several times, leaving a 'texture' of visited and non-visited sites inside this box.

To control this texture we split the random walk trajectory into what we call 'excursions' which correspond to the successive visits to $A$. Using some classical results from random walk theory, we will establish three key facts about these excursions:

(i) the successive excursions to $A$ are roughly independent from each other,

(ii) the first point in $A$ visited by each excursion has a limiting distribution (as $N$ grows), which we call the normalized equilibrium distribution on $A$.

(iii) when the number of steps $n$ scales as $u N^{d}$, the number of excursions entering $A$ is approximately Poisson distributed (with parameter is proportional to $u$ ).

All these properties hold precisely in the limit $N \rightarrow \infty$ when $A$ is kept fixed.

Starting from these three properties of the random walk excursions, we define a measure on $\{0,1\}^{A}$, which is the candidate for the asymptotic distribution of the wanted infinite volume percolation (intersected with $A$ ). This limiting distribution is what we call the local picture. In view of properties (i)-(iii), it should not be surprising that Poisson point processes enter the construction here.

In Chapter 3. we will use this local picture to give a definition of random interlacements. To this end we map $A \subset \mathbb{T}_{N}^{d}$ into its isometric copy $\mathbb{A} \subset$ $\mathbb{Z}^{d}$ and transfer the local picture to $\{0,1\}^{\mathbb{A}}$. Then, taking the limit as $\mathbb{A} \uparrow \mathbb{Z}^{d}$, we essentially obtain a consistent measure on $\{0,1\}^{\mathbb{Z}^{d}}$, which we call random interlacements.

The actual construction of random interlacements given in Chapter 3 is more complicated, because its goal is not only to prove the existence (and uniqueness) of the infinite volume measure, but also to provide a way to perform calculations. In particular, the actual construction aims on preserving the Poisson point process structure appearing in the local picture.

We will construct the random interlacements as a trace left on $\mathbb{Z}^{d}$ by a Poisson point process on the space of doubly infinite random walk trajectories modulo time shift, see (3.10) below. Intuitively speaking, every trajectory appearing in this Poisson point process correspond to one excursion of the random walk in the torus.

In Chapter 4 after having defined the random interlacements measure, we will describe some of its basic properties. We will study its correlations, 
translation invariance and ergodicity, and compare it to Bernoulli site percolation. This comparison helps determining which of the techniques that have already been developed for Bernoulli percolation have chance to work in the random interlacements setting.

As we will find out, many of the techniques of Bernoulli percolation are not directly applicable for random interlacements. Therefore, we will need to adapt them, or develop new techniques that are robust enough to deal with dependence. In Chapter 5 , we prove a result related to the existence of a phase transition for random interlacements on high dimensions, see Theorem 5.2. The proof of this theorem makes use of a technique which is very important in various contexts, namely the multi-scale renormalization.

The last two chapters of these notes present two recent branches of the random interlacements research. In Chapter 6, we study the properties of the interlacement set of random interlacements on $\mathbb{Z}^{d}$. This set is the complement of the vacant set, and should be viewed as the limit of occupied/corroded sites in the random-walk-on-torus problem. We will see that the properties of the interlacement set are rather well understood, contrary to those of the vacant set.

Finally, in Chapter 7, we return to random walk on finite graphs, this time however not on the torus, but on random regular graphs. We explain that the infinite volume limit of this problem is random interlacements on regular trees, and sketch the techniques used to show that for these graphs there is a phase transition in the spirit of 1.5 .

We would like to precise the scope and structure of these notes. We do not want to present a comprehensive reference of what is currently known about random interlacements. Instead, we intend to favor a motivated and self-contained exposition, with more detailed proofs of basic facts that should give the reader familiarity with the tools needed to work on the subject. The results presented here are not the most precise currently available, instead they were chosen in a way to balance between simplicity and relevance. For interested reader we collect the pointers to the relevant literature at the end of each chapter. 


\section{Chapter 2}

\section{Random walk on the torus}

In this chapter we discuss some properties of the random walk on a discrete torus. Our aim is to motivate the definition of the local picture discussed in the Introduction, that is to understand the intersection of the trajectory of the random walk with a fixed set $A \subset \mathbb{T}_{N}^{d}$ as $N$ becomes large.

\section{$2.1 \quad$ Notation}

Let us start by fixing the notation used through these notes. We consider, for $N \geq 1$, the discrete torus $\mathbb{T}_{N}^{d}=(\mathbb{Z} / N \mathbb{Z})^{d}$, which we regard as a graph with an edge connecting two vertices if and only if their Euclidean distance is one.

On $\mathbb{T}_{N}^{d}$ we consider a simple random walk. For technical reasons that will be explained later, we actually consider the so called lazy random walk which stays put, with probability one half, and jumps otherwise to a uniformly chosen neighbor. Its transition matrix is given by

$$
C(x, y)= \begin{cases}1 / 2, & \text { if } x=y \\ 1 /(4 d), & \text { if } x \text { and } y \text { are neighbors in } \mathbb{T}_{N}^{d}, \\ 0, & \text { otherwise }\end{cases}
$$

Let $\pi$ be the uniform distribution on the torus $\mathbb{T}_{N}^{d}$. It is easy to see that $\pi$ and $C(x, y)$ satisfy the detailed balance condition, that is $\pi$ is reversible for the random walk.

We write $P$ for the law on $\left(\mathbb{T}_{N}^{d}\right)^{\mathbb{N}}$ of lazy simple random walk on $\mathbb{T}_{N}^{d}$ started with uniform distribution $\pi$. We let $X_{n}, n \geq 0$, stand for the canonical process on $\left(\mathbb{T}_{N}^{d}\right)^{\mathbb{N}}$. The law of the canonical (lazy) random walk started at a specified point $x \in \mathbb{T}_{N}^{d}$ is denoted by $P_{x}$. 
Note that we omit the dependence on $N$ in the notation $\pi, P, P_{x}$ and $X_{n}$. This will be done in other situations throughout the text, hoping that the context will clarify the omission.

For $k \geq 0$, we introduce the canonical shift operator $\theta_{k}$ in the space of trajectories $\left(\mathbb{T}_{N}^{d}\right)^{\mathbb{N}}$, which is characterized by $X_{n} \circ \theta_{k}=X_{n+k}$ for every $n \geq 0$. Analogously, we can define $\theta_{T}$ for a random time $T$.

The main reason for considering the lazy random walk are the following facts:

$C(\cdot, \cdot)$ has only positive eigenvalues $1=\lambda_{1}>\lambda_{2} \geq \cdots \geq \lambda_{N^{d}} \geq 0$.

Its spectral gap $\Lambda_{N}:=\lambda_{1}-\lambda_{2}$ satisfies $\Lambda_{N} \geq c N^{-2}$,

see for instance [29], Theorems 5.5 and 12.4. A simple calculation using the spectral decomposition leads then to

$$
\sup _{x, y \in \mathbb{T}_{N}^{d}}\left|P_{x}\left[X_{n}=y\right]-\pi(y)\right| \leq c e^{-\Lambda_{N} n}, \text { for all } n \geq 0,
$$

see [29, (4.22), (4.35) and Theorem 5.5.

It will be useful to define the regeneration time $r_{N}$ associated to the simple random walk on $\mathbb{T}_{N}^{d}$ by

$$
r_{N}=\Lambda_{N}^{-1} \log ^{2} N \sim c N^{2} \log ^{2} N .
$$

To justify the name regeneration time, observe that, for every $x \in \mathbb{T}_{N}^{d}$, by (2.2) and (2.4), the total variation distance between $P_{x}\left[X_{r_{N}} \in \cdot\right]$ and $\pi$ satisfies

$$
\begin{aligned}
\left\|P_{x}\left[X_{r_{N}} \in \cdot\right]-\pi(\cdot)\right\|_{\mathrm{TV}} & :=\frac{1}{2} \sum_{y \in \mathbb{T}_{N}^{d}}\left|P_{x}\left[X_{r_{N}}=y\right]-\pi(y)\right| \\
& \leq c^{\prime} N^{d} e^{-c \log ^{2} N} \\
& \leq c^{\prime} e^{-c \log ^{2} N}
\end{aligned}
$$

which decays with $N$ faster than any polynomial. This means that, independently of its starting distribution, the distribution of the random walk position at time $r_{N}$ is very close to being uniform.

We also consider the simple (lazy) random walk on the infinite lattice $\mathbb{Z}^{d}$ where edges again connect points with Euclidean distance one. The canonical law of this random walk starting at some point $x \in \mathbb{Z}^{d}$ is denoted by $P_{x}^{\mathbb{Z}^{d}}$. If no confusion may arise, we write simply $P_{x}$.

We introduce the entrance and hitting times $H_{A}$ and $\tilde{H}_{A}$ of a set $A$ of vertices in $\mathbb{T}_{N}^{d}$ (or in $\mathbb{Z}^{d}$ ) by

$$
\begin{aligned}
& H_{A}=\inf \left\{t \geq 0: X_{t} \in A\right\}, \\
& \tilde{H}_{A}=\inf \left\{t \geq 1: X_{t} \in A\right\} .
\end{aligned}
$$


Throughout these notes, we will suppose that the dimension $d$ is greater or equal to three (cf. Remark 1.1), implying that

$$
\text { the random walk on } \mathbb{Z}^{d} \text { is transient. }
$$

Fix now a finite set $\mathbb{A} \subset \mathbb{Z}^{d}$ (usually we will denote subsets of $\mathbb{Z}^{d}$ by $\mathbb{A}, \mathbb{B}, \ldots)$. Due to the transience of the random walk, we can define the equilibrium measure, $e_{\mathbb{A}}$, and the capacity, $\operatorname{cap}(\mathbb{A})$, of $\mathbb{A}$ by

$$
\begin{aligned}
e_{\mathbb{A}}(x) & :=\mathbf{1}_{x \in \mathbb{A}} P_{x}^{\mathbb{Z}^{d}}\left[\tilde{H}_{\mathbb{A}}=\infty\right], \quad x \in \mathbb{Z}^{d}, \\
\operatorname{cap}(\mathbb{A}) & :=e_{\mathbb{A}}(\mathbb{A})=e_{\mathbb{A}}\left(\mathbb{Z}^{d}\right) .
\end{aligned}
$$

Note that $\operatorname{cap}(\mathbb{A})$ normalizes the measure $e_{\mathbb{A}}$ into a probability distribution.

Throughout this notes we use $B(x, r)$ to denote the closed ball centered at $x$ with radius $r$ (in the graph distance), considered as a subset of $\mathbb{Z}^{d}$ or $\mathbb{T}_{N}^{d}$, depending on the context.

\subsection{Local entrance point}

We now start the study of the local picture left by the random walk on $\mathbb{T}_{N}^{d}$. To this end, consider a (finite) box $\mathbb{A} \subset \mathbb{Z}^{d}$ centered at the origin. For each $N$ larger than the diameter of $\mathbb{A}$, one can find a copy $A_{N}$ of this box inside $\mathbb{T}_{N}^{d}$. We are interested in the distribution of the intersection of the random walk trajectory (run up to time $n$ ) with the set $A_{N}$, that is $\left\{X_{0}, X_{1}, \ldots, X_{n}\right\} \cap A_{N}$. As $N$ increases, the boxes $A_{N}$ get much smaller compared to the whole torus $\mathbb{T}_{N}^{d}$, explaining the use of the terminology 'local picture'. In particular, it is easy to see that

$$
\pi\left(A_{N}\right) \rightarrow 0 \quad \text { as } \quad N \rightarrow \infty
$$

As soon as $N$ is strictly larger than the diameter of the box $\mathbb{A}$, we can find an isomorphism $\phi_{N}: A_{N} \rightarrow \mathbb{A}$ between the box $A_{N}$ and its copy $\mathbb{A}$ in the infinite lattice. As usual, to avoid a clumsy notation, we will drop the indices $N$ by $\phi_{N}$ and $A_{N}$.

The first question we attempt to answer concerns the distribution of the point where the random walk typically enters the box $A$. Our goal is to show that this distribution almost does not depend on the starting point of the walk, if it starts far enough from the box $A$.

To specify what we mean by 'far enough from $A$ ', we consider a sequence of boxes $\mathbb{A}_{N}^{\prime}$ centered at the origin in $\mathbb{Z}^{d}$ and having diameter $N^{1 / 2}$ (the specific value $1 / 2$ is not particularly important, any value strictly between zero and one would work for our purposes here). Note that for $N$ large enough $\mathbb{A}_{N}^{\prime}$ contains $\mathbb{A}$ and $N^{1 / 2} \leq N$. Therefore, we can extend the isomorphism $\phi$ defined above to $\phi: A_{N}^{\prime} \rightarrow \mathbb{A}_{N}^{\prime} \subset \mathbb{Z}^{d}$, where $A_{N}^{\prime}$ is a copy 
of $\mathbb{A}_{N}^{\prime}$ inside $\mathbb{T}_{N}^{d}$. Also $\pi\left(A_{N}^{\prime}\right) \rightarrow 0$ as $N \rightarrow \infty$, therefore, under $P$, the random walk typically starts outside of $A_{N}^{\prime}$.

The first step in determination of the entrance distribution to $A$ is the following lemma which roughly states that the random walk likely regenerates before hitting $A$.

Lemma 2.1. $(d \geq 3)$ For $A^{\prime}$ and $\mathbb{A}^{\prime}$ as above, there exists $\delta>0$ such that

$$
\begin{aligned}
& \sup _{x \in \mathbb{T}_{N}^{d} \backslash A^{\prime}} P_{x}\left[H_{A} \leq r_{N}\right] \leq N^{-\delta}, \text { 'A isn't hit before regenerating' } \\
& \sup _{x \in \mathbb{Z}^{d} \backslash \mathbb{A}_{N}^{\prime}} P_{x}^{\mathbb{Z}^{d}}\left[H_{\mathbb{A}}<\infty\right] \leq N^{-\delta} \text {, 'escape to infinity before hitting } \mathbb{A}^{\prime},
\end{aligned}
$$

Proof. From [27, Proposition 1.5.10] it can be easily deduced that there is $c<\infty$ such that for any $\ell \geq 1$ and $x \in \mathbb{Z}^{d}$, with $|x|>\ell$,

$$
P_{x}^{\mathbb{Z}^{d}}\left[H_{B(0, \ell)}<\infty\right] \leq c\left(\frac{\ell}{|x|}\right)^{d-2} .
$$

The estimate 2.12 then follows directly from $(2.13)$.

To show 2.11), let $\Pi$ be the canonical projection from $\mathbb{Z}^{d}$ onto $\mathbb{T}_{N}^{d}$. Given an $x$ in $\mathbb{T}_{N}^{d} \backslash A^{\prime}$, we can bound $P_{x}\left[H_{A} \leq r_{N}\right]$ from above by

$$
P_{\phi(x)}^{\mathbb{Z}^{d}}\left[H_{B\left(\phi(x), N \log ^{2} N\right)^{c}} \leq r_{N}\right]+P_{\phi(x)}^{\mathbb{Z}^{d}}\left[H_{\Pi^{-1}(A) \cap B\left(\phi(x), N \log ^{2} N\right)}<\infty\right] .
$$

Using (3.30) on p. 227 of [31, we obtain

$$
\begin{aligned}
P_{\phi(x)}^{\mathbb{Z}^{d}} & {\left[H_{B\left(\phi(x), N \log ^{2} N\right)^{c}} \leq r_{N}\right] } \\
& \leq P_{\phi(x)}^{\mathbb{Z}^{d}}\left[\max _{1 \leq t \leq r_{N}}\left|X_{t}-\phi(x)\right|_{\infty} \geq c N \log ^{2} N\right] \\
& \leq 2 d \exp \left\{-2\left(c N \log ^{2} N\right)^{2} / 4 r_{N}\right\} \\
& \leq c e^{-c \log ^{2} N} .
\end{aligned}
$$

The set $\Pi^{-1}(A) \cap B\left(\phi(x), N \log ^{2} N\right)$ is contained in a union of no more than $c \log ^{c} N$ translated copies of the set $\mathbb{A}$. By the choice of $x, \phi(x)$ is at distance at least $c N^{1 / 2}$ from each of these copies. Hence, using the union bound and 2.13 again, we obtain that

$$
P_{\phi(x)}\left[H_{\Pi^{-1}(A) \cap B\left(\phi(x), N \log ^{2} N\right)}<\infty\right] \leq c(\log N)^{c} N^{-c} .
$$

Inserting the last two estimates into 2.14, we have shown 2.11).

As a consequence of (2.11), we can now show that, up to a typically small error, the probability $P_{y}\left[X_{H_{A}}=x\right]$ does not depend much on the starting point $y \in \mathbb{T}_{N}^{d} \backslash A^{\prime}$ : 


\section{Lemma 2.2.}

$$
\sup _{\substack{x \in A, y^{\prime} \in \mathbb{T}_{N}^{d} \backslash A^{\prime}}}\left|P_{y}\left[X_{H_{A}}=x\right]-P_{y^{\prime}}\left[X_{H_{A}}=x\right]\right| \leq c N^{-\delta} .
$$

Proof. We apply the following intuitive argument: by the previous lemma, it is unlikely that the random walk started at $y \in \mathbb{T}_{N}^{d} \backslash A^{\prime}$ visits the set $A$ before time $r_{N}$, and at time $r_{N}$ the distribution of the random walk is already close to uniform, i.e. it is independent of $y$. Therefore the hitting distribution cannot depend on $y$ too much.

To make this intuition into a proof, we first observe that 2.17 is implied by

$$
\sup _{y \in \mathbb{T}_{N}^{d} \backslash A^{\prime}}\left|P_{y}\left[X_{H_{A}}=x\right]-P\left[X_{H_{A}}=x\right]\right| \leq c N^{-\delta} .
$$

To show 2.18, we first deduce from inequality 2.4 that

$$
\begin{aligned}
& \sup _{y \in \mathbb{T}_{N}^{d} \backslash A^{\prime}}\left|E_{y}\left[P_{X_{r_{N}}}\left[X_{H_{A}}=x\right]\right]-P\left[X_{H_{A}}=x\right]\right| \\
& \quad \leq \sum_{y^{\prime} \in \mathbb{T}_{N}^{d}} \sup _{y \in \mathbb{T}_{N}^{d} \backslash A^{\prime}}\left|P_{y}\left[X_{r_{N}}=y^{\prime}\right]-\pi\left(y^{\prime}\right)\right| P_{y^{\prime}}\left[X_{H_{A}}=x\right] \\
& \leq c N^{d} e^{-c \log ^{2} N} \leq e^{-c \log ^{2} N} .
\end{aligned}
$$

For any $y \in \mathbb{T}_{N}^{d} \backslash A^{\prime}$, by the simple Markov property applied at time $r_{N}$ and the estimate 2.19 ,

$$
\begin{aligned}
P_{y}\left[X_{H_{A}}=x\right] & \leq P_{y}\left[X_{H_{A}}=x, H_{A}>r_{N}\right]+P_{y}\left[H_{A} \leq r_{N}\right] \\
& \leq E_{y}\left[P_{X_{r_{N}}}\left[X_{H_{A}}=x\right]\right]+P_{y}\left[H_{A} \leq r_{N}\right] \\
& \leq P\left[X_{H_{A}}=x\right]+e^{-c \log ^{2} N}+P_{y}\left[H_{A} \leq r_{N}\right] .
\end{aligned}
$$

With (2.11), we have therefore shown that for any $y \in \mathbb{T}_{N}^{d} \backslash A^{\prime}$,

$$
P_{y}\left[X_{H_{A}}=x\right]-P\left[X_{H_{A}}=x\right] \leq N^{-\delta} .
$$

On the other hand, for any $y \in \mathbb{T}_{N}^{d} \backslash A^{\prime}$, by the simple Markov property at time $r_{N}$ again,

$$
\begin{aligned}
P_{y}\left[X_{H_{A}}=x\right] & \geq P_{y}\left[X_{H_{A}}=x, H_{A}>r_{N}\right] \\
& \geq E_{y}\left[P_{X_{r_{N}}}\left[X_{H_{A}}=x\right]\right]-P_{y}\left[H_{A} \leq r_{N}\right] \\
& \geq P\left[X_{H_{A}}=x\right]-N^{-\delta}
\end{aligned}
$$

using 2.19, 2.11 in the last inequality. Combining 2.21 and 2.22, 2.18 follows. 
Given that the distribution of the entrance point of the random walk in $A$ is roughly independent of the starting point (given that the starting point is not in $A^{\prime}$ ), we are naturally tempted to determine such distribution. This is the content of the next lemma, which will play an important role in motivating the definition of random interlacements later.

Lemma 2.3. For $A$ and $A^{\prime}$ as above there is $\delta>0$ such that

$$
\sup _{x \in A, y \in \mathbb{T}_{N}^{d} \backslash A^{\prime}}\left|P_{y}\left[X_{H_{A}}=x\right]-\frac{e_{\mathbb{A}}(\phi(x))}{\operatorname{cap}(\mathbb{A})}\right| \leq N^{-\delta} .
$$

Note that the entrance law is approximated by the (normalized) exit distribution, cf. definition $(2.9)$ of the equilibrium measure. This is intimately related to the reversibility of the random walk.

Proof. Let us fix vertices $x \in A, y \in \mathbb{T}_{N}^{d} \backslash A^{\prime}$. We first define the equilibrium measure of $A$, with respect to the random walk killed when exiting $A^{\prime}$,

$$
e_{A}^{A^{\prime}}(z)=\mathbf{1}_{A}(z) P_{z}\left[H_{\mathbb{T}_{N}^{d} \backslash A^{\prime}}<\tilde{H}_{A}\right] \text {, for any } z \in A \text {. }
$$

Note that by 2.12 and the strong Markov property applied at $H_{\mathbb{T}_{N}^{d} \backslash A^{\prime}}$,

$$
e_{\mathbb{A}}(\phi(z)) \leq e_{A}^{A^{\prime}}(z) \leq e_{\mathbb{A}}(\phi(z))+N^{-\delta}, \text { for any } z \in A
$$

In order to make the expression $P_{y}\left[X_{H_{A}}=x\right]$ appear, we consider the probability that the random walk started at $x$ escapes from $A$ to $\mathbb{T}_{N}^{d} \backslash A^{\prime}$ and then returns to the set $A$ at some point other than $x$. By reversibility of the random walk with respect to the measure $\left(\pi_{z}\right)_{z \in \mathbb{T}_{N}^{d}}$, we have

$$
\begin{aligned}
\sum_{z \in A \backslash\{x\}} \pi_{x} P_{x}\left[H_{\mathbb{T}_{N}^{d} \backslash A^{\prime}}\right. & \left.<\tilde{H}_{A}, X_{\tilde{H}_{A}}=z\right]=\pi_{x} P_{x}\left[H_{\mathbb{T}_{N}^{d} \backslash A^{\prime}}<\tilde{H}_{A}, X_{\tilde{H}_{A}} \neq x\right] \\
& =\sum_{z \in A \backslash\{x\}} \pi_{z} P_{z}\left[H_{\mathbb{T}_{N}^{d} \backslash A^{\prime}}<\tilde{H}_{A}, X_{\tilde{H}_{A}}=x\right]
\end{aligned}
$$

By the strong Markov property applied at time $H_{\mathbb{T}_{N}^{d} \backslash A^{\prime}}$, we have for any $z \in A$,

$$
\begin{aligned}
\pi_{z} P_{z}\left[H_{\mathbb{T}_{N}^{d} \backslash A^{\prime}}\right. & \left.<\tilde{H}_{A}, X_{\tilde{H}_{A}}=x\right] \\
& =\pi_{z} E_{z}\left[\mathbf{1}_{\left\{H_{\mathbb{T}_{N}^{d} \backslash A^{\prime}}<\tilde{H}_{A}\right\}} P_{X_{H_{\mathbb{T}_{N}^{d} \backslash A^{\prime}}}}\left[X_{H_{A}}=x\right]\right] .
\end{aligned}
$$

With 2.25 and 2.17), this yields

$$
\left|\pi_{z} P_{z}\left[H_{\mathbb{T}_{N}^{d} \backslash A^{\prime}}<\tilde{H}_{A}, X_{\tilde{H}_{A}}=x\right]-\pi_{x} e_{\mathbb{A}}(\phi(z)) P_{y}\left[X_{H_{A}}=x\right]\right| \leq N^{-\delta},
$$


for any $z \in A$. With this estimate applied to both sides of (2.26), we obtain

$$
\begin{aligned}
& \pi_{x} e_{\mathbb{A}}(\phi(x))\left(1-P_{y}\left[X_{H_{A}}=x\right]\right) \\
&=P_{y}\left[X_{H_{A}}=x\right]\left(\pi_{x} \operatorname{cap}(\mathbb{A})-\pi_{x} e_{\mathbb{A}}(\phi(x))\right)+O\left(|A| N^{-\delta}\right),
\end{aligned}
$$

implying (2.23).

We observe that the entrance distribution $P_{y}\left[X_{H_{B}}=\cdot\right]$ was approximated in Lemma 2.3 by a quantity that is independent of $N$ and solely relates to the infinite lattice random walk. This is a very important ingredient of the local picture construction.

\subsection{Local measure}

We continue to study the trace that a random walk on the torus leaves inside a small box $A \subset \mathbb{T}_{N}^{d}$. We already know from the previous section that the random walk typically enters $A$ in a point $x$ chosen with distribution $e_{\mathbb{A}}(\phi(x)) / \operatorname{cap}(\mathbb{A})$. After entering the box $A$, the random walk behaves in the same way as on the infinite lattice $\mathbb{Z}^{d}$ until it gets far away from $A$ again. We will therefore split the random walk trajectory into so-called excursions. For this, recall the definition of $A^{\prime}$ and the shift operators $\theta_{k}$ from Section 2.2 and let

$$
\begin{array}{ll}
R_{0}=H_{A}, & D_{0}=H_{\mathbb{T}_{N}^{d} \backslash A^{\prime}} \circ \theta_{R_{0}}+R_{0}, \\
R_{l}=H_{A} \circ \theta_{D_{l-1}}+D_{l-1}, & D_{l}=H_{\mathbb{T}_{N}^{d} \backslash A^{\prime}} \circ \theta_{R_{l}}+R_{l}, \quad \text { for } l \geq 1 .
\end{array}
$$

These will be respectively called return and departure times of the random walk between $A$ and $A^{\prime}$, see Figure 2.1 .

Observe that every time $n$ for which the random walk is inside $A$ has to satisfy $R_{k} \leq n<D_{k}$ for some $k \geq 0$. This implies that

$$
\left\{X_{0}, X_{1}, \ldots, X_{D_{k}}\right\} \cap A=\bigcup_{j=0}^{k}\left\{X_{R_{j}}, X_{R_{j}+1}, \ldots, X_{D_{j}}\right\} \cap A .
$$

Or in other words, the trace left by the random walk trajectory in $A$ up to time $D_{k}$ is given by union of the traces of $k$ separate excursions.

Since $X_{D_{k}} \notin A_{N}^{\prime}$, using Lemma 2.2 and the strong Markov property applied at the time $D_{k}$, we can conclude that the set of points in $A$ visited by the random walk between times $R_{k+1}$ and $D_{k+1}$ is roughly independent of what happened before the time $D_{k}$. Therefore, the excursions $\left\{X_{R_{j}}, X_{R_{j}+1}, \ldots, X_{D_{j}}\right\}, j=0,1,2, \ldots$, should be roughly independent. A fixed number of such excursions should actually become i.i.d. in the limit $N \rightarrow \infty$. 


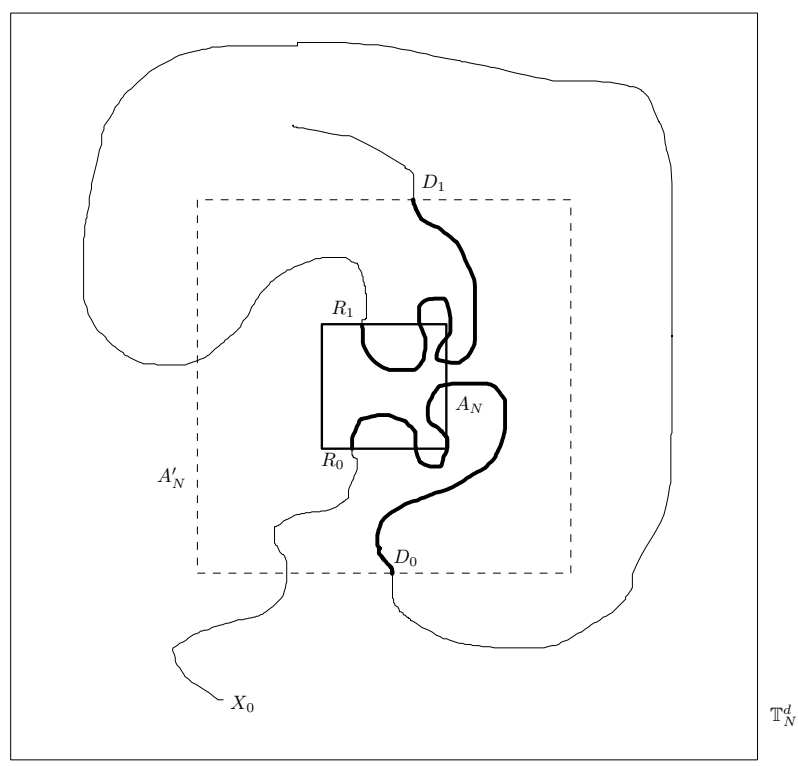

Figure 2.1: A trajectory of the random walk inside the torus split into excursions (thick lines) and the remaining parts (thin lines) with respect to the boxes $A_{N}, A_{N}^{\prime}$.

Lemma 2.3 yields that the entrance points $X_{R_{j}}$ of these trajectories in $A$ are asymptotically distributed as $e_{\mathbb{A}}(\phi(\cdot)) / \operatorname{cap}(\mathbb{A})$. Moreover, as $N$ grows, the difference $D_{k}-R_{k}$ tends to infinity. Therefore, as $N$ grows, the excursion $\left\{X_{R_{j}+1}, \ldots, X_{D_{j}}\right\}$ looks more and more like a simple random walk trajectory on $\mathbb{Z}^{d}$ (note that this heuristic claim is only true because the random walk on $\mathbb{Z}^{d}$, for $d \geq 3$, is transient).

From the previous arguments it follows that the asymptotic distribution of every excursion should be given by

$$
\bar{Q}_{\mathbb{A}}\left[X_{0}=x,\left(X_{n}\right)_{n \geq 0} \in \cdot\right]=\frac{e_{\mathbb{A}}(x)}{\operatorname{cap}(\mathbb{A})} P_{x}^{\mathbb{Z}^{d}}[\cdot], \text { for } x \in \mathbb{Z}^{d}
$$

To understand fully the trace left by random walk in $A$, we now have to understand how many excursions are typically performed by the random walk between $A$ and $A^{\prime}$ until some fixed time $n$.

Using a reversibility argument again, we first compute the expected number of excursions before time $n$. To this end fix $k \geq 0$ and a and let us estimate the probability that $k$ is a return time $R_{j}$ for some $j \geq 0$. This 
probability can be written as

$$
\begin{aligned}
P & {[k} \\
& \left.=R_{j} \text { for some } j \geq 0\right] \\
& =\sum_{x \in A} \sum_{y \in\left(A^{\prime}\right)^{c}} \sum_{m \leq k} P\left[X_{m}=y, X_{k}=x, X_{i} \in A^{\prime} \backslash A, m<i<k\right] .
\end{aligned}
$$

Let $\Gamma_{j}(y, x)$ be the set of possible random walk trajectories of length $j$ joining $y$ to $x$ and staying in $A^{\prime} \backslash A$ between times 1 and $j-1$. By reversibility, for every $\gamma \in \Gamma_{j}(y, x)$ and $l \geq 0$,

$$
\begin{aligned}
P\left[\left(X_{l}, \ldots, X_{l+j}\right)=\gamma\right] & =\pi(y) P_{y}\left[\left(X_{0}, \ldots, X_{j}\right)=\gamma\right] \\
& =\pi(x) P_{x}\left[\left(X_{0}, \ldots, X_{j}\right)=\hat{\gamma}\right],
\end{aligned}
$$

where $\hat{\gamma} \in \Gamma_{j}(x, y)$ is the time-reversed path $\gamma$. Observing that the time reversal is a bijection from $\Gamma_{j}(y, x)$ to $\Gamma_{j}(x, y)$, the right-hand side of 2.33 can be written as

$$
\begin{aligned}
& \sum_{m=0}^{k} \sum_{x \in A} \sum_{y \in\left(A^{\prime}\right)^{c}} \sum_{\gamma \in \Gamma_{k-m}(y, x)} P\left[\left(X_{m}, \ldots, X_{k}\right)=\gamma\right] \\
& \stackrel{2.34}{-} \sum_{m=0}^{k} \sum_{x \in A} \sum_{y \in\left(A^{\prime}\right)^{c}} \sum_{\gamma \in \Gamma_{k-m}(x, y)} \pi(x) P_{x}\left[\left(X_{0}, \ldots, X_{k-m}\right)=\gamma\right] \\
& =\sum_{x \in A} \sum_{m=0}^{k} N^{-d} P_{x}\left[k-m=H_{\mathbb{T}_{N}^{d} \backslash A^{\prime}}<\tilde{H}_{A}\right] \\
& =N^{-d} \sum_{x \in A} P_{x}\left[H_{\mathbb{T}_{N}^{d} \backslash A^{\prime}}<\min \left\{k, \tilde{H}_{A}\right\}\right] .
\end{aligned}
$$

We now use 2.25 to obtain that

$$
\lim _{N \rightarrow \infty} \lim _{k \rightarrow \infty} \mid N^{d} P\left[k=R_{j} \text { for some } j \geq 0\right]-\operatorname{cap} \mathbb{A} \mid=0 .
$$

As the random variables $\left(R_{i+1}-R_{i}\right)_{i \geq 0}$ are asymptotically i.i.d. with non-periodic support, we expect by the renewal theory that

$$
\lim _{N \rightarrow \infty} N^{-d} E\left[R_{i+1}-R_{i}\right]=(\operatorname{cap} \mathbb{A})^{-1},
$$

see for instance [18] Example 3.3 p. 292 and Exercise 4.2 p.301. Thus, for all $u>0$ fixed,

$$
\lim _{N \rightarrow \infty} E\left[\# \text { excursions between times } 0 \text { and } u N^{d}\right]=u \operatorname{cap} \mathbb{A} \text {. }
$$

Remark that we finally obtained a justification for the scaling mentioned in 1.2$)$ ! 
The expectation of the difference $R_{i+1}-R_{i} \sim N^{d}$ is much larger than the regeneration time. Hence, typically the random walk regenerates many times before returning to $A$. It is thus plausible that, asymptotically as $N \rightarrow \infty, N^{-d}\left(R_{i+1}-R_{i}\right) /$ cap $\mathbb{A}$ has exponential distribution with parameter 1 , and that the number of excursions before $u N^{d}$ has Poisson distribution with parameter cap $\mathbb{A}$. This heuristic can be made rigorous, see [1, 2].

Combining the discussion of the previous paragraph with the asymptotic i.i.d. property of the excursions, and with 2.32 , we deduce the following somewhat informal description of how the random walk visits $A$ :

- the random walk trajectory is split into roughly independent excursions,

- for each $x \in A$, the number of excursions starting at $x$ is approximately an independent Poisson random variable with mean $u e_{\mathbb{A}}(x)$,

- the trace left by the random walk on $A$ is given by the union of all these excursions intersected with $A$.

To make the last point slightly more precise, observe that with high probability $X_{0}, X_{u N^{d}} \notin A^{\prime}$, that the times 0 and $u N^{d}$ are not in any excursion. So with high probability there are no incomplete excursion in $A$ at time $u N^{d}$.

\subsection{Local picture as Poisson point process}

We are now going to make the above informal construction precise, using the formalism of Poisson point processes. For this, let us first introduce some notation. Let $W_{+}$be the space of infinite random walk trajectories that spend only a finite time in finite sets of $\mathbb{Z}^{d}$.

$$
\begin{array}{r}
W_{+}=\left\{w: \mathbb{N} \rightarrow \mathbb{Z}^{d}:\|w(n)-w(n+1)\|_{1} \leq 1 \text { for each } n \geq 0\right. \\
\text { and } \left.\{n: w(n)=y\} \text { is finite for all } y \in \mathbb{Z}^{d}\right\} .
\end{array}
$$

(Recall that we consider lazy random walk.) As usual, $X_{n}, n \geq 0$, denote the canonical coordinates on $W_{+}$defined by $X_{n}(w)=w(n)$. We endow the space $W_{+}$with the $\sigma$-algebra $\mathcal{W}_{+}$generated by the coordinate maps $X_{n}, n \geq 0$.

Recall the definition of $\bar{Q}_{\mathbb{A}}$ in 2.32 and define the measure $Q_{\mathbb{A}}^{+}:=$ $\operatorname{cap}(\mathbb{A}) \bar{Q}_{\mathbb{A}}$ on $\left(W^{+}, \mathcal{W}^{+}\right)$, that is

$$
Q_{\mathbb{A}}^{+}\left[X_{0}=x,\left(X_{n}\right)_{n \geq 0} \in F\right]=e_{\mathbb{A}}(x) P_{x}^{\mathbb{Z}^{d}}[F], \quad x \in \mathbb{Z}^{d}, F \in \mathcal{W}^{+} .
$$

From the transience of the simple random walk on $\mathbb{Z}^{d}$ it follows that $W_{+}$ has full measure under $Q_{\mathbb{A}}^{+}$, that is $Q_{\mathbb{A}}^{+}\left(W_{+}\right)=e_{\mathbb{A}}(\mathbb{A})=\operatorname{cap} \mathbb{A}$. 
To define the Poisson point process we consider the space of finite point measures on $W^{+}$,

$$
\Omega_{+}=\left\{\omega_{+}=\sum_{i=1}^{n} \delta_{w_{i}}: n \in \mathbb{N}, w_{1}, \ldots, w_{n} \in W_{+}\right\},
$$

where $\delta_{w}$ stands for the Dirac's measure on $w$. We endow this space with the $\sigma$-algebra generated by the evaluation maps $\omega_{+} \mapsto \omega_{+}(D)$, where $D \in \mathcal{W}_{+}$.

Now let $\mathbb{P}_{\AA}^{u}$ be the law of a Poisson point process on $\Omega_{+}$with intensity measure $u Q_{\AA}^{+}$(see e.g. [39], Proposition 3.6, for the definition and the construction). The informal description given at the end of the last section can be then retranslated into following theorem. Its full proof, completing the sketchy arguments of the last section, can be found in [56].

Theorem 2.4. Let $u>0, J$ be the index of the last excursion started before $u N^{d}, J:=\max \left\{i: R_{i} \leq u N^{d}\right\}$. For every $i \leq J$ define $w_{i}^{N} \in W^{+}$ to be an (arbitrary) extension of the $i^{\text {th }}$ excursion, that is

$$
w_{i}^{n}(k)=\phi\left(X_{R_{i}+k}\right) \quad \text { for all } k \in\left\{0, \ldots, D_{i}-R_{i}\right\} .
$$

Then the distribution of the point process $\sum_{i \leq J} \delta_{w_{i}^{N}}$ converges to $\mathbb{P}_{\mathbb{A}}^{u}$ weakly on $\Omega_{+}$.

As consequence, the distribution of $\phi\left(\left\{X_{0}, \ldots, X_{u N^{d}}\right\} \cap A\right)$ on $\{0,1\}^{\mathbb{A}}$ converges to the distribution of $\bigcup_{i \leq N}$ Range $w_{i} \cap \mathbb{A}$, where the law of $\omega_{+}=$ $\sum_{i=1}^{N} \delta_{w_{i}}$ is given by $\mathbb{P}_{\mathbb{A}}^{u}$.

\section{$2.5 \quad$ Notes}

The properties of the vacant set left by the random walk on the torus were for the first time studied by Benjamini and Sznitman in [6]. In this paper it is shown that (for $d$ large enough) when the number of steps scales as $n=u N^{d}$ for $u$ sufficiently small, then the vacant set has a giant component, which is unique (in a weak sense). The size of the second largest component was studied in 55. . Other studies have been concerned with the geometric aspects of the trace of a random walk on the torus, such as 32 and 34 .

The local picture in the torus and its connection with random interlacements was established in [56] for many distant microscopic boxes simultaneously. This result was largely improved in [53, by extending the connection to mesoscopic boxes of size $N^{1-\varepsilon}$. More precisely, for $A$ being a box of size $N^{1-\varepsilon}$ in the torus, Theorem 1.1 of [53] constructs a coupling between the random walk on the torus and random interlacements at levels $u(1-\varepsilon)$ and $u(1+\varepsilon)$ such that for arbitrary $\alpha>0$

$$
\operatorname{Prob}\left[\mathcal{I}^{(1-\varepsilon) u} \cap A \subset\left\{X_{0}, \ldots, X_{u N^{d}}\right\} \cap \mathbb{A} \subset \mathcal{I}^{(1+\varepsilon) u} \cap A\right] \geq 1-N^{-\alpha},
$$


for $N$ large enough, depending on $d, \epsilon, u$ and $\alpha$. Here, $\mathcal{I}^{u}$ is the interlacement set at level $u$, that we will construct in the next chapter.

This coupling allows one to prove the best known results on the properties of the largest connected component $\mathcal{C}_{\max }(u, N)$ of the vacant set on the torus, going in direction of the phase transition mentioned in (1.5). In the following theorem, which is taken from Theorems 1.2-1.4 of [53], $u_{\star}=u_{\star}(d)<\infty$ denotes the critical parameter of random interlacements on $\mathbb{Z}^{d}$, that we define in (4.34) below, and $u_{\star \star}=u_{\star \star}(d)<\infty$ is another critical value introduced in (0.6) of [46], satisfying $u_{\star \star} \geq u_{\star}$. We recommend 41 for further material on this subject.

Theorem $2.5(d \geq 3)$. $\quad$ (i) Subcritical phase: When $u>u_{\star}$, then for every $\eta>0$,

$$
P\left[\left|\mathcal{C}_{\max }(u, N)\right| \geq \eta N^{d}\right] \stackrel{N \rightarrow \infty}{\longrightarrow} 0 .
$$

In addition, when $u>u_{\star \star}$, then for some $\lambda>0$

$$
P\left[\left|\mathcal{C}_{\max }(u, N)\right| \geq \log ^{\lambda} N\right] \stackrel{N \rightarrow \infty}{\longrightarrow} 0 .
$$

(ii) Supercritical phase: When $u$ is small enough then for some $\delta>0$,

$$
P\left[\left|\mathcal{C}_{\max }(u, N)\right| \geq \delta N^{d}\right] \stackrel{N \rightarrow \infty}{\longrightarrow} 1 .
$$

Moreover, for $d \geq 5$, the second largest component of the vacant set has size at most $\log ^{\lambda} N$ with high probability.

It is believed that the assumption $u>u_{\star}$ of 2.44 is optimal, i.e. 2.44 does not hold for any $u \leq u_{\star}$. The other two results are not so optimal. The following behavior is conjectured:

Conjecture 2.6. The vacant set of the random walk on the torus exhibits a phase transition. Its critical threshold coincides with the critical value $u_{\star}$ of random interlacements on $\mathbb{Z}^{d}$. In addition, $u_{\star \star}=u_{\star}$ and thus for $u>u_{\star}$, 2.45 holds. Finally, for $u<u_{\star}$

$$
N^{-d}\left|\mathcal{C}_{\max }(u, N)\right| \stackrel{N \rightarrow \infty}{\longrightarrow} \rho(u) \in(0,1) .
$$

\subsubsection{Disconnection of a discrete cylinder}

These notes would be incomplete without mentioning another problem which motivated the introduction of random interlacements: the disconnection of a discrete cylinder, or, in a more picturesque language, the problem of 'termite in a wooden beam'.

In this problem one considers a discrete cylinder $G \times \mathbb{Z}$, where $G$ is an arbitrary finite graph, most prominent example being the torus $\mathbb{T}_{N}^{d}, d \geq 2$. On the cylinder one considers a simple random walk started from a point 
in its base, $G \times\{0\}$. The object of interest is the disconnection time, $T_{G}$, of the discrete cylinder which is the first time such that the range of the random walk disconnects the cylinder. More precisely, $T_{G}$ is the smallest time such that, for a large enough $M,(-\infty,-M] \times G$ and $[M, \infty) \times G$ are contained in two distinct connected components of the complement of the range, $(G \times \mathbb{Z}) \backslash\left\{X_{0}, \ldots, X_{T_{G}}\right\}$.

The study of this problem was initiated by Dembo and Sznitman [16]. It is shown there that $T_{N}:=T_{\mathbb{T}_{N}^{d}}$, is of order $N^{2 d}$, on the logarithmic scale:

$$
\lim _{N \rightarrow \infty} \frac{\log T_{N}}{\log N}=2 d, \quad d \geq 2 .
$$

This result was successively improved in [17] (a lower bound on $T_{N}$ : the collection of random variables $N^{2 d} / T_{N}, N \geq 1$, is tight when $d \geq 17$ ), 44 (the lower bound hold for any $d \geq 2$ ), 46] (an upper bound: $T_{N} / N^{2 d}$ is tight). Disconnection time of cylinders with a general base $G$ is studied in [43]: for the class of bounded degree bases $G$, it is shown that $T_{G}$ is roughly of oder $|G|^{2}$.

Some of the works cited in the last paragraph explore considerably the connection of the problem with the random interlacements. This connection was established in [45], and states that the local picture left by random walk on the discrete cylinder converges locally to random interlacements. The connection is slightly more complicated than on the torus (that is why we choose the torus for our motivation). The complication comes from the fact that the parameter $u$ of the limiting random interlacements is not deterministic but random, and depends on the local time of the 'vertical projection' of the random walk. We state the connection as a theorem which is a simplified version of [45, Theorem 0.1].

Theorem 2.7. Let $x_{N} \in \mathbb{T}_{N}^{d} \times \mathbb{Z}$ be such that its $\mathbb{Z}$-component $z_{N}$ satisfies $\lim _{N \rightarrow \infty} z_{N} / N^{d}=v$. Let $L_{t}^{z}=\sum_{i=0}^{t} 1\left\{X_{i} \in \mathbb{T}_{N}^{d} \times\{z\}\right\}$ be the local time of the vertical projection of the random walk. Assume that $t_{N}$ satisfies $\lim t_{N} / N^{2 d}=\alpha$. Then, for any $n>0$ fixed, in distribution,

$$
\left(\left\{X_{0}, \ldots, X_{t_{N}}\right\} \cap B\left(x_{N}, n\right), L_{t_{N}}^{z_{N}} / N^{d}\right) \stackrel{N \rightarrow \infty}{\longrightarrow}\left(\mathcal{I}^{L} \cap B(n), L\right),
$$

where $L /(d+1)$ has the distribution of the local time of Brownian motion at time $\alpha /(d+1)$ and spatial position $v$, and $\mathcal{I}^{L}$ is the interlacement set of random interlacements at level $L$.

A version of Theorem 2.7 for cylinders with general base $G$ is given in 57 .

The dependence of the intensity of the random interlacements on the local time of the vertical projection should be intuitively obvious: While in the horizontal direction the walk mixes rather quickly (in time $N^{2} \log N$ ), 
there is no averaging going on in vertical direction. Therefore the intensity of the local picture around $x_{N}$ must depend on the time that the random walk spends in the layer $z_{N}$, which is given by $L_{t_{N}}^{z_{N}}$.

It should be not surprising that Conjecture 2.6 can be transfered to the disconnection problem:

Conjecture 2.8 (Remark 4.7 of [46]). The random variable $T_{N} / N^{2 d}$ converges in distribution to a random variable $U$ which is defined by

$$
U=\inf \left\{t \geq 0: \sup _{x \in \mathbb{R}} \ell(t /(d+1), x) \geq u_{\star}(d+1)\right\}
$$

where $\ell(t, x)$ is the local time of a one-dimensional Brownian motion and $u_{\star}(d+1)$ is the critical value of random interlacements on $\mathbb{Z}^{d+1}$.

A relation between the tails of $T_{N} / N^{2 d}$ and of the random variable $U$ is given in [46]. 


\section{Chapter 3}

\section{Definition of random interlacements}

The goal of this chapter is to extend the local picture obtained previously, cf. Theorem 2.4 to the whole lattice. We will define a (dependent) percolation model on $\mathbb{Z}^{d}$, called random interlacements, whose restriction to any finite set $\mathbb{A} \subset \mathbb{Z}^{d}$ is given by (the trace of) the Poisson point process $\mathbb{P}_{\mathbb{A}}^{u}$.

\subsection{Construction of random interlacements}

Before starting the real construction, let us first sketch a cheap argument for the existence of the infinite volume limit of the local pictures (it is worth remarking that infinite volume limit refers here to $\mathbb{A} \uparrow \mathbb{Z}^{d}$, not to the limit $N \rightarrow \infty$ performed in the last chapter). To this end consider $A \subset \mathbb{T}_{N}^{d}$, and denote by $Q_{A}^{N, u}$ the distribution of trace left by random walk in $A$, that is the distribution of $\left(\mathbf{1}\left\{x \in \mathcal{V}_{N}\left(u N^{d}\right)\right\}\right)_{x \in A}$ on $\{0,1\}^{A}$. Consider now another set $\bar{A} \supset A$ and $N \geq \operatorname{diam} \bar{A}$. From the definition, it is obvious that the measures $Q_{A}^{N, u}$ and $Q_{\bar{A}}^{N, u}$ automatically satisfy the restriction property

$$
Q_{A}^{N, u}=\pi_{\bar{A}, A} \circ Q_{\bar{A}}^{N, u}, 1
$$

where $\pi_{\bar{A}, A}:\{0,1\}^{\bar{A}} \rightarrow\{0,1\}^{A}$ is the usual restriction map. Moreover, by Theorem 2.4 (or Theorem 1.1 of [56]),

$$
Q_{A}^{N, u} \text { converges weakly as } N \rightarrow \infty \text { to a measure } Q_{A}^{u},
$$

\footnotetext{
${ }^{1}$ For a measurable map $f: S_{1} \rightarrow S_{2}$ and a measure $\mu$ on $S_{1}$, we use $f \circ \mu$ to denote the push forward of $\mu$ by $f,(f \circ \mu)(\cdot):=\mu\left(f^{-1}(\cdot)\right)$.
} 
where $Q_{A}^{u}$ is the distribution of the trace left on $A$ by the Poisson process $\mathbb{P}_{A}^{u}$.

Using 3.2 , we can see that the restriction property 3.1 passes to the limit, that is

$$
Q_{A}^{u}=\pi_{\bar{A}, A} \circ Q_{\bar{A}}^{u}
$$

Kolmogorov's extension theorem then yields the existence of the infinite volume measure $Q^{u}$ on $\{0,1\} \mathbb{Z}^{d}$ (endowed with the usual cylinder $\sigma$-field).

The construction of the previous paragraph has a considerable disadvantage. First, it relies on 3.2 , whose proof is partly sketchy in these notes. Secondly, it does not give enough information about the measure $Q^{u}$. In particular, we completely lost the nice feature that $Q_{A}^{u}$ is the trace of a Poisson point process of random walk trajectories.

This is the motivation for another, more constructive, definition of the infinite volume model. The reader might consider this definition rather technical. However, the effort put into it will be more than paid back when working with the model. The following construction follows the original paper [4] with minor modifications.

We wish to construct the infinite volume analog to the Poisson point process $\mathbb{P}_{\mathbb{A}}^{u}$. The first step is to introduce the measure space where the new Poisson point process will be defined. To this end we need few definitions.

Similarly to 2.39), let $W$ be the space of doubly-infinite random walk trajectories that spend only a finite time in finite subsets of $\mathbb{Z}^{d}$, i.e.

$$
\begin{array}{r}
W=\left\{w: \mathbb{Z} \rightarrow \mathbb{Z}^{d}:\|w(n)-w(n+1)\|_{1} \leq 1 \text { for each } n \geq 0\right. \\
\text { and } \left.\{n: w(n)=y\} \text { is finite for all } y \in \mathbb{Z}^{d}\right\} .
\end{array}
$$

We again denote with $X_{n}, n \in \mathbb{Z}$, the canonical coordinates on $W$, and write $\theta_{k}, k \in \mathbb{Z}$, for the canonical shifts,

$$
\theta_{k}(w)(\cdot)=w(\cdot+k), \text { for } k \in \mathbb{Z} \quad\left(\text { resp. } k \geq 0 \text { when } w \in W_{+}\right) .
$$

We endow $W$ with the $\sigma$-algebra $\mathcal{W}$ generated by the canonical coordinates.

Given $\mathbb{A} \subset \mathbb{Z}^{d}, w \in W$ (resp. $w \in W_{+}$), we define the entrance time in $\mathbb{A}$ and the exit time from $\mathbb{A}$ for the trajectory $w$ :

$$
\begin{aligned}
& H_{\mathbb{A}}(w)=\inf \left\{n \in \mathbb{Z}(\operatorname{resp} . \mathbb{N}): X_{n}(w) \in \mathbb{A}\right\}, \\
& T_{\mathbb{A}}(w)=\inf \left\{n \in \mathbb{Z}(\operatorname{resp} . \mathbb{N}): X_{n}(w) \notin \mathbb{A}\right\} .
\end{aligned}
$$

When $\mathbb{A} \subset \subset \mathbb{Z}^{d}$ (i.e. $\mathbb{A}$ is a finite subset of $\mathbb{Z}^{d}$ ), we consider the subset of $W$ of trajectories entering $\mathbb{A}$ :

$$
W_{\mathbb{A}}=\left\{w \in W: X_{n}(w) \in \mathbb{A} \text { for some } n \in \mathbb{Z}\right\} .
$$


We can write $W_{\mathbb{A}}$ as a countable partition into measurable sets

$$
W_{\mathbb{A}}=\bigcup_{n \in \mathbb{Z}} W_{\mathbb{A}}^{n}, \quad \text { where } W_{\mathbb{A}}^{n}=\left\{w \in W: H_{\mathbb{A}}(w)=n\right\} .
$$

Heuristically, the reason why we need to work with the space $W$ of the doubly-infinite trajectories is that when taking the limit $\mathbb{A} \rightarrow \mathbb{Z}^{d}$, the 'excursions' start at infinity.

The first step of the construction of the random interlacements is to extend the measure $Q_{\mathbb{A}}^{+}$to the space $W$. This is done, naturally, by requiring that $\left(X_{-n}\right)_{n>0}$ is a simple random walk started at $X_{0}$ conditioned not to return to $\mathbb{A}$. More precisely, we define on $(W, \mathcal{W})$ the measure $Q_{\mathbb{A}}$ by

$$
Q_{\mathbb{A}}\left[\left(X_{-n}\right)_{n \geq 0} \in F, X_{0}=x,\left(X_{n}\right)_{n \geq 0} \in G\right]=P_{x}\left[F \mid \widetilde{H}_{\mathbb{A}}=\infty\right] e_{\mathbb{A}}(x) P_{x}[G],
$$

for $F, G \in \mathcal{W}_{+}$and $x \in \mathbb{Z}^{d}$.

Observe that $Q_{\mathbb{A}}$ gives full measure to $W_{\mathbb{A}}^{0}$. This however means that the set $\mathbb{A}$ is still somehow registered in the trajectories, more precisely the origin of the time is at the first visit to $\mathbb{A}$. To solve this issue, it is convenient to consider the space $W^{\star}$ of trajectories in $W$ modulo time shift

$$
W^{\star}=W / \sim \text {, where } w \sim w^{\prime} \text { iff } w(\cdot)=w^{\prime}(\cdot+k) \text { for some } k \in \mathbb{Z},
$$

which allows us to 'ignore' the rather arbitrary (and $\mathbb{A}$-dependent) time parametrization of the random walks. We denote with $\pi^{\star}$ the canonical projection from $W$ to $W^{\star}$. The map $\pi^{\star}$ induces a $\sigma$-algebra in $W^{\star}$ given by

$$
\mathcal{W}^{\star}=\left\{U \subset W^{\star}:\left(\pi^{\star}\right)^{-1}(U) \in \mathcal{W}\right\} .
$$

It is the largest $\sigma$-algebra on $W^{\star}$ for which $(W, \mathcal{W}) \stackrel{\pi^{\star}}{\rightarrow}\left(W^{\star}, \mathcal{W}^{\star}\right)$ is measurable. We use $W_{\mathbb{A}}^{\star}$ to denote the set of trajectories modulo time shift entering $\mathbb{A} \subset \mathbb{Z}^{d}$,

$$
W_{\mathbb{A}}^{\star}=\pi^{\star}\left(W_{\mathbb{A}}\right) .
$$

It is easy to see that $W_{\mathbb{A}}^{\star} \in \mathcal{W}^{\star}$.

The random interlacements process that we are defining will be governed by a Poisson point process on the space $\left(W^{\star} \times \mathbb{R}_{+}, \mathcal{W}^{\star} \otimes \mathcal{B}\left(\mathbb{R}_{+}\right)\right)$. To this end we define $\Omega$ in analogy to 2.41$)$ :

$$
\begin{aligned}
& \Omega=\left\{\omega=\sum_{i \geq 1} \delta_{\left(w_{i}^{\star}, u_{i}\right)}: w_{i}^{\star} \in W^{\star}, u_{i} \in \mathbb{R}_{+}\right. \text {such that } \\
&\left.\quad \omega\left(W_{\mathbb{A}}^{\star} \times[0, u]\right)<\infty, \text { for every } \mathbb{A} \subset \subset \mathbb{Z}^{d} \text { and } u \geq 0\right\} .
\end{aligned}
$$

This space is endowed with the $\sigma$-algebra $\mathcal{A}$ generated by the evaluation maps $\omega \mapsto \omega(D)$ for $D \in \mathcal{W}^{\star} \otimes \mathcal{B}\left(\mathbb{R}_{+}\right)$. 
At this point, the reader may ask why we do not take $\Omega$ to be simply the space of point measures on $W^{\star}$. The reason for this is that we are, for practical reasons, trying to construct the infinite volume limit of local pictures for all values of parameter $u \geq 0$ simultaneously. In other words, we construct a coupling of random interlacements models for different values of $u$, similar to the usual coupling of Bernoulli percolation measures with different values of parameter $p$. The component $u_{i}$ of the couple $\left(w_{i}^{\star}, u_{i}\right)$ can be viewed as a label attached to the trajectory $w_{i}^{\star}$. This trajectory will influence the random interlacements model at level $u$ only if its label satisfy $u_{i} \leq u$.

The intensity measure of the Poisson point process governing the random interlacements will be given by $\nu \otimes \mathrm{d} u$. Here, $\mathrm{d} u$ is the Lebesgue measure on $\mathbb{R}_{+}$and the measure $\nu$ on $W^{\star}$ is constructed as an appropriate extension of $Q_{\mathbb{A}}$ to $W^{\star}$ in the following theorem.

Theorem 3.1 (47, Theorem 1.1). There exists a unique $\sigma$-finite measure $\nu$ on the space $\left(W^{\star}, \mathcal{W}^{\star}\right)$ satisfying, for each finite set $\mathbb{A} \subset \mathbb{Z}^{d}$,

$$
1_{W_{\mathbb{A}}^{\star}} \cdot \nu=\pi^{\star} \circ Q_{\mathbb{A}},{ }^{2}
$$

where the finite measure $Q_{\mathbb{A}}$ on $W_{\mathbb{A}}$ is given by 3.9 .

Proof. The uniqueness of $\nu$ satisfying 3.14 is clear since, given a sequence of sets $\mathbb{A}_{k} \uparrow \mathbb{Z}^{d}, W^{\star}=\cup_{k} W_{\mathbb{A}_{k}}^{\star}$.

For the existence, what we need to prove is that, for fixed $\mathbb{A} \subset \mathbb{A}^{\prime} \subset \mathbb{Z}^{d}$,

$$
\pi^{\star} \circ\left(1_{W_{\mathbb{A}}} \cdot Q_{\mathbb{A}^{\prime}}\right)=\pi^{\star} \circ Q_{\mathbb{A}} .
$$

Indeed, we can then set, for arbitrary $\mathbb{A}_{k} \uparrow \mathbb{Z}^{d}$,

$$
\nu=\sum_{k} 1_{W_{\mathbb{A}_{k}}^{\star} \backslash W_{\mathbb{A}_{k-1}}^{\star}} \cdot\left(\pi^{\star} \circ Q_{\mathbb{A}_{k}}\right) .
$$

The equality $(3.15)$ then insures that $\nu$ does not depend on the sequence $A_{k}$.

We introduce the space

$$
W_{\mathbb{A}, \mathbb{A}^{\prime}}=\left\{w \in W_{\mathbb{A}}: H_{\mathbb{A}^{\prime}}(w)=0\right\}
$$

and the bijection $s_{\mathbb{A}, \mathbb{A}^{\prime}}: W_{\mathbb{A}, \mathbb{A}^{\prime}} \rightarrow W_{\mathbb{A}, \mathbb{A}}$ given by

$$
\left[s_{\mathbb{A}, \mathbb{A}^{\prime}}(w)\right](\cdot)=w\left(H_{\mathbb{A}}(w)+\cdot\right),
$$

moving the origin of time from the entrance to $\mathbb{A}^{\prime}$ to the entrance to $\mathbb{A}$.

To prove 3.15 , it is enough to show that

$$
s_{\mathbb{A}, \mathbb{A}^{\prime}} \circ\left(1_{W_{\mathbb{A}, \mathbb{A}^{\prime}}} \cdot Q_{\mathbb{A}^{\prime}}\right)=Q_{\mathbb{A}} \cdot
$$

\footnotetext{
${ }^{2}$ For any set $G$ and measure $\nu$, we define $1_{G} \cdot \nu(\cdot):=\nu(G \cap \cdot)$.
} 
Indeed, from 3.9 it follows that $1_{W_{\mathbb{A}, \mathbb{A}^{\prime}}} \cdot Q_{\mathbb{A}^{\prime}}=1_{W_{\mathbb{A}}} \cdot Q_{\mathbb{A}^{\prime}}$ and thus 3.15 follows just by applying $\pi^{\star}$ on both sides (3.19).

To show (3.19), we consider the set $\Sigma$ of finite paths $\sigma:\left\{0, \ldots, N_{\sigma}\right\} \rightarrow$ $\mathbb{Z}^{d}$ such that $\sigma(0) \in \mathbb{A}^{\prime}, \sigma(n) \notin \mathbb{A}$ for $n<N_{\sigma}$, and $\sigma\left(N_{\sigma}\right) \in \mathbb{A}$. We split the left-hand side of 3.19 by partitioning $W_{\mathbb{A}, \mathbb{A}^{\prime}}$ into the sets

$$
W_{\mathbb{A}, \mathbb{A}^{\prime}}^{\sigma}=\left\{w \in W_{\mathbb{A}, \mathbb{A}^{\prime}}: w \text { restricted to }\left\{0, \cdots, N_{\sigma}\right\} \text { equals } \sigma\right\} \text {, for } \sigma \in \Sigma \text {. }
$$

For $w \in W_{\mathbb{A}, \mathbb{A}^{\prime}}^{\sigma}$, we have $H_{\mathbb{A}}(w)=N_{\sigma}$, so that we can write

$$
s_{\mathbb{A}, \mathbb{A}^{\prime}} \circ\left(1_{W_{\mathbb{A}, \mathbb{A}^{\prime}}} \cdot Q_{\mathbb{A}^{\prime}}\right)=\sum_{\sigma \in \Sigma} \theta_{N_{\sigma}} \circ\left(1_{W_{\mathbb{A}, \mathbb{A}^{\prime}}^{\sigma}} \cdot Q_{\mathbb{A}^{\prime}}\right) .
$$

To prove 3.19 , consider an arbitrary collection of sets $A_{i} \subset \mathbb{Z}^{d}$, for $i \in \mathbb{Z}$, such that $A_{i} \neq \mathbb{Z}^{d}$ for at most finitely many $i \in \mathbb{Z}$. Then,

$$
\begin{aligned}
s_{\mathbb{A}, \mathbb{A}^{\prime}} & \circ\left(1_{W_{\mathbb{A}, \mathbb{A}^{\prime}}} \cdot Q_{\mathbb{A}^{\prime}}\right)\left[X_{i} \in A_{i}, i \in \mathbb{Z}\right] \\
= & \sum_{\sigma \in \Sigma} Q_{\mathbb{A}^{\prime}}\left[X_{i+N_{\sigma}}(w) \in A_{i}, i \in \mathbb{Z}, w \in W_{\mathbb{A}, \mathbb{A}^{\prime}}^{\sigma}\right] \\
= & \sum_{\sigma \in \Sigma} Q_{\mathbb{A}^{\prime}}\left[X_{i}(w) \in A_{i-N_{\sigma}}, i \in \mathbb{Z}, w \in W_{\mathbb{A}, \mathbb{A}^{\prime}}^{\sigma}\right] .
\end{aligned}
$$

Using formula 3.9 , the identity

$$
e_{\mathbb{A}}(x) P_{x}\left[\cdot \mid \widetilde{H}_{\mathbb{A}}=\infty\right]=P_{x}\left[\cdot, \widetilde{H}_{\mathbb{A}}=\infty\right], \quad x \in \operatorname{supp} e_{\mathbb{A}^{\prime}},
$$

and the Markov property, the above expression equals

$$
\begin{aligned}
& \sum_{x \in \operatorname{supp}} \sum_{\mathbb{A}^{\prime}} P_{x \in \Sigma}\left[X_{j} \in A_{-j-N_{\sigma}}, j \geq 0, \widetilde{H}_{\mathbb{A}^{\prime}}=\infty\right] \\
& \quad \times P_{x}\left[X_{n}=\sigma(n) \in A_{n-N_{\sigma}}, 0 \leq n \leq N_{\sigma}\right] \\
& \quad \times P_{\sigma\left(N_{\sigma}\right)}\left[X_{n} \in A_{n}, n \geq 0\right] \\
& =\sum_{x \in \operatorname{supp} e_{\mathbb{A}^{\prime}}} \sum_{y \in \mathbb{A}} \sum_{\sigma: \sigma\left(N_{\sigma}\right)=y} P_{x}\left[X_{j} \in A_{-j-N_{\sigma}}, j \geq 0, \widetilde{H}_{\mathbb{A}^{\prime}}=\infty\right] \\
& \quad \times P_{x}\left[X_{n}=\sigma(n) \in A_{n-N_{\sigma}}, 0 \leq n \leq N_{\sigma}\right] P_{y}\left[X_{n} \in A_{n}, n \geq 0\right] .
\end{aligned}
$$

For fixed $x \in \operatorname{supp} e_{\mathbb{A}^{\prime}}$ and $y \in \mathbb{A}$, we have, using the reversibility in the 
first step and the Markov property in the second,

$$
\begin{aligned}
& \sum_{\sigma: \sigma\left(N_{\sigma}\right)=y} P_{x}\left[X_{j} \in A_{-j-N_{\sigma}}, j \geq 0, \widetilde{H}_{\mathbb{A}^{\prime}}=\infty\right] \\
& \quad \times P_{x}\left[X_{n}=\sigma(n) \in A_{n-N_{\sigma}}, 0 \leq n \leq N_{\sigma}\right] \\
& =\sum_{\substack{\sigma: \sigma\left(N_{\sigma}\right)=y \\
\sigma(0)=x}} P_{x}\left[X_{j} \in A_{-j-N_{\sigma}}, j \geq 0, \widetilde{H}_{\mathbb{A}^{\prime}}=\infty\right] \\
& \quad \times P_{y}\left[X_{m}=\sigma\left(N_{\sigma}-m\right) \in A_{-m}, 0 \leq m \leq N_{\sigma}\right] \\
& =\sum_{\substack{\sigma: \sigma\left(N_{\sigma}\right)=y \\
\sigma(0)=x}} P_{y}\left[\begin{array}{l}
\left.X_{m}=\sigma\left(N_{\sigma}-m\right) \in A_{-m}, 0 \leq m \leq N_{\sigma},\right] \\
=P_{y}\left[\begin{array}{l}
\tilde{H}_{\mathbb{A}}=\infty, m \geq N_{\sigma}, \widetilde{H}_{\mathbb{A}^{\prime}} \circ \theta_{N_{\sigma}}=\infty \\
\text { occurs at } x, X_{m} \in A_{-m}, m \geq 0
\end{array}\right]
\end{array}\right.
\end{aligned}
$$

Using 3.25 in 3.24 and summing over $x \in \operatorname{supp} e_{\mathbb{A}^{\prime}}$, we obtain

$$
\begin{aligned}
s_{\mathbb{A}, \mathbb{A}^{\prime}} & \circ\left(1_{W_{\mathbb{A}, \mathbb{A}^{\prime}}} \cdot Q_{\mathbb{A}^{\prime}}\right)\left[X_{i} \in A_{i}, i \in \mathbb{Z}\right] \\
& =\sum_{y \in \mathbb{A}} P_{y}\left[\widetilde{H}_{\mathbb{A}}=\infty, X_{m}=A_{-m}, m \geq 0\right] P_{y}\left[X_{m} \in A_{m}, m \geq 0\right] \\
& \stackrel{3.9}{=} Q_{\mathbb{A}}\left[X_{m} \in A_{m}, m \in \mathbb{Z}\right] .
\end{aligned}
$$

This shows (3.19) and concludes the proof of the existence of a measure $\nu$ satisfying (3.14). Moreover, the measure $\nu$ is clearly $\sigma$-finite, it is sufficient to observe that $\nu\left(W_{\mathbb{A}}^{\star},[0, u]\right)<\infty$ for any $\mathbb{A} \subset \subset \mathbb{Z}^{d}$ and $u \geq 0$.

We can now complete the construction of the random interlacements model by describing the infinite volume of the local pictures discussed in the previous chapter. On the space $(\Omega, \mathcal{A})$ we consider the law $\mathbb{P}$ of a Poisson point process with intensity $\nu\left(\mathrm{d} w^{\star} \otimes \mathrm{d} u\right)$ (recall that $\nu$ is $\sigma$-finite). With the usual identification of point measures and subsets, under $\mathbb{P}$, the configuration $\omega$ can be viewed as an infinite random cloud of doubly-infinite random walk trajectories (modulo time-shift) with attached non-negative labels $u_{i}$.

Finally, for $\omega=\sum_{i>0} \delta_{\left(w_{i}^{\star}, u_{i}\right)} \in \Omega$ we define two subsets of $\mathbb{Z}^{d}$, the interlacement set at level $u$, that is the set of sites visited by the trajectories with label smaller than $u$,

$$
\mathcal{I}^{u}(\omega)=\bigcup_{i: u_{i} \leq u} \operatorname{Range}\left(w_{i}^{\star}\right),
$$


and its complement, the vacant set at level $u$,

$$
\mathcal{V}^{u}(\omega)=\mathbb{Z}^{d} \backslash \mathcal{I}^{u}(\omega)
$$

Let $\Pi^{u}$ be the mapping from $\Omega$ to $\{0,1\}^{\mathbb{Z}^{d}}$ given by

$$
\Pi^{u}(\omega)=\left(\mathbf{1}\left\{x \in \mathcal{V}^{u}(\omega)\right\}: x \in \mathbb{Z}^{d}\right) .
$$

We endow the space $\{0,1\}^{\mathbb{Z}^{d}}$ with the $\sigma$-field $\mathcal{Y}$ generated by the canonical coordinates $\left(Y_{x}: x \in \mathbb{Z}^{d}\right)$. As for $\mathbb{A} \subset \subset \mathbb{Z}^{d}$, we have

$$
\mathcal{V}^{u} \supset \mathbb{A} \quad \text { if and only if } \quad \omega\left(W_{\mathbb{A}}^{\star} \times[0, u]\right)=0,
$$

the mapping $\Pi^{u}:(\Omega, \mathcal{A}) \rightarrow\left(\{0,1\}^{\mathbb{Z}^{d}}, \mathcal{Y}\right)$ is measurable. We can thus define on $\left(\{0,1\}^{\mathbb{Z}^{d}}, \mathcal{Y}\right)$ the law $Q^{u}$ of the vacant set at level $u$ by

$$
Q^{u}=\Pi^{u} \circ \mathbb{P} .
$$

The law $Q^{u}$ of course coincides with the law $Q^{u}$ constructed abstractly using Kolmogorov's theorem below (3.3). In addition, we however gained a rather rich structure behind it, and which will be useful later.

Some additional notation. We close this chapter by introducing some additional notation that we use frequently through the remaining chapters. Let $s_{\mathbb{A}}: W_{\mathbb{A}}^{\star} \rightarrow W$ be defined as

$s_{\mathbb{A}}\left(w^{\star}\right)=w^{0}$, where $w^{0}$ is the unique element of $W_{\mathbb{A}}^{0}$ with $\pi^{\star}\left(w^{0}\right)=w^{\star}$,

i.e. $s_{\mathbb{A}}$ 'gives to $w \in W_{A}^{\star}$ its $\mathbb{A}$-dependent time parametrization'. We also define a measurable map $\mu_{\mathbb{A}}$ from $\Omega$ to the space of point measures on $\left(W_{+} \times \mathbb{R}_{+}, \mathcal{W}_{+} \otimes \mathcal{B}\left(\mathbb{R}_{+}\right)\right)$via

$$
\mu_{\mathbb{A}}(\omega)(f)=\int_{W_{\mathbb{A}}^{\star} \times \mathbb{R}_{+}} f\left(s_{\mathbb{A}}\left(w^{\star}\right)_{+}, u\right) \omega\left(\mathrm{d} w^{\star}, \mathrm{d} u\right), \quad \text { for } \omega \in \Omega,
$$

where $f$ is a non-negative measurable function on $W_{+} \times \mathbb{R}_{+}$and for $w \in$ $W, w_{+} \in W_{+}$is its restriction to $\mathbb{N}$. In words, $\mu_{\mathbb{A}}$ selects from $\omega$ those trajectories that hit $\mathbb{A}$ and erases their parts prior to the first visit to $\mathbb{A}$. We further define a measurable function $\mu_{\mathbb{A}, u}$ from $\Omega$ to the space of point measures on $\left(W_{+}, \mathcal{W}_{+}\right)$by

$$
\mu_{\mathbb{A}, u}(\omega)(\mathrm{d} w)=\mu_{\mathbb{A}}(\omega)(\mathrm{d} w \times[0, u]),
$$

which 'selects' from $\mu_{\mathbb{A}}(\omega)$ only those trajectories whose labels are smaller than $u$. Observe that

$$
\mathcal{I}^{u}(\omega) \cap \mathbb{A}=\bigcup_{w \in \operatorname{supp} \mu_{\mathbb{A}, u}(\omega)} \text { Range } w \cap \mathbb{A} .
$$


It also follows from the construction of the measure $\mathbb{P}$ and from the defining property (3.14) of $\nu$ that

$$
\mu_{\mathbb{A}, u} \circ \mathbb{P}=\mathbb{P}_{\mathbb{A}}^{u} .
$$

\subsection{Notes}

As we mentioned, random interlacements on $\mathbb{Z}^{d}$ were first time introduced in [47. Later, 50] extended the construction of the model to any transient weighted graphs. Since then, large effort has been spent in the study of its percolative and geometrical properties, which relate naturally to the above mentioned questions on the fragmentation of a torus by random walk. In the next chapter we start to study some of the most basic properties of this model on $\mathbb{Z}^{d}$. 


\section{Chapter 4}

\section{Properties of random interlacements}

\subsection{Basic properties}

We now study the random interlacements model introduced in the last chapter. Our first goal is to understand the correlations present in the model.

To state the first result we define the Green's function of the random walk,

$$
g(x, y)=\sum_{n \geq 0} P_{x}\left[X_{n}=y\right], \text { for } x, y \in \mathbb{Z}^{d} .
$$

We write $g(x)$ for $g(x, 0)$, and refer to [27], Theorem 1.5.4 p.31 for the following estimate

$$
\frac{c^{\prime}}{1+|x-y|^{d-2}} \leq g(x, y) \leq \frac{c}{|x-y|^{d-2}}, \text { for } x, y \in \mathbb{Z}^{d} .
$$

Lemma 4.1. For every $u \geq 0, x, y \in \mathbb{Z}^{d}, \mathbb{A} \subset \subset \mathbb{Z}^{d}$,

$$
\begin{aligned}
& \mathbb{P}\left[\mathbb{A} \subset \mathcal{V}^{u}\right]=\exp \{-u \operatorname{cap}(\mathbb{A})\}, \\
& \mathbb{P}\left[x \in \mathcal{V}^{u}\right]=\exp \{-u / g(0)\}, \\
& \mathbb{P}\left[\{x, y\} \in \mathcal{V}^{u}\right]=\exp \left\{-\frac{2 u}{g(0)+g(y-x)}\right\}
\end{aligned}
$$

Remark 4.2. The equality 4.3 in fact characterizes the distribution of the vacant set $\mathcal{V}^{u}$, and can be used to define the measure $Q^{u}$. This follows from the theory of point processes, see e.g. [25], Theorem 12.8(i). 
Proof. Using the notation introduced at the end of the last chapter, we observe that $\mathbb{A} \subset \mathcal{V}^{u}(\omega)$ if and only if $\mu_{\mathbb{A}, u}(\omega)=0$. Claim (4.3) then follows from

$$
\begin{aligned}
\mathbb{P}\left[\mu_{\mathbb{A}, u}(\omega)=0\right] \stackrel{3.36}{-} \exp \left\{-u Q_{\mathbb{A}}\left(W_{+}\right)\right\} \\
\stackrel{2.40}{-} \exp \left\{-u e_{\mathbb{A}}\left(\mathbb{Z}^{d}\right)\right\}=\exp \{-u \operatorname{cap}(\mathbb{A})\} .
\end{aligned}
$$

The remaining claims of the lemma follows from (4.3), once we compute $\operatorname{cap}(\{x\})$ and $\operatorname{cap}(\{x, y\})$. For the first case, observe that under $P_{x}$ the number of visits to $x$ has geometrical distribution with parameter $P_{x}\left[\tilde{H}_{x}=\infty\right]=\operatorname{cap}(\{x\})$, by the strong Markov property. This yields immediately that

$$
\operatorname{cap}(\{x\})=g(0)^{-1} .
$$

For the second case, we recall the useful formula that we prove later,

$$
P_{x}\left[H_{\mathbb{A}}<\infty\right]=\sum_{y \in \mathbb{A}} g(x, y) e_{\mathbb{A}}(y), \quad x \in \mathbb{Z}^{d}, \mathbb{A} \subset \subset \mathbb{Z}^{d} .
$$

Assuming without loss of generality that $x \neq y$, we write $e_{\{x, y\}}=\rho_{x} \delta_{x}+$ $\rho_{y} \delta_{y}$, and $\operatorname{cap}(\{x, y\})=\rho_{x}+\rho_{y}$ for some $\rho_{x}, \rho_{y} \geq 0$. From formula 4.8, it follows that

$$
1=\rho_{x} g(z, x)+\rho_{y} g(z, y), \quad \text { for } z \in\{x, y\} .
$$

Solving this system for $\rho_{x}, \rho_{y}$ yields

$$
\operatorname{cap}(\{x, y\})=\frac{2}{g(0)+g(x-y)} .
$$

Claims (4.4) and (4.5) then follows directly from (4.3) and (4.7), (4.10).

To show (4.8), let $L=\sup \left\{k \geq 0: X_{k} \in \mathbb{A}\right\}$ be the time of the last visit to $\mathbb{A}$, with convention $L=-\infty$ if $\mathbb{A}$ is not visited. Then,

$$
\begin{aligned}
P_{x}\left[H_{\mathbb{A}}<\infty\right] & =P_{x}[L \geq 0]=\sum_{y \in \mathbb{A}} \sum_{n \geq 0} P_{x}\left[L=n, X_{L}=y\right] \\
& =\sum_{y \in \mathbb{A}} \sum_{n \geq 0} P_{x}\left[X_{n}=y, X_{k} \notin \mathbb{A} \text { for } k>n\right] \\
& =\sum_{y \in \mathbb{A}} \sum_{n \geq 0} P_{x}\left[X_{n}=y\right] e_{\mathbb{A}}(y)=\sum_{y \in \mathbb{A}} g(x, y) e_{\mathbb{A}}(y),
\end{aligned}
$$

where we used the strong Markov property in the forth, and the definition of the Green function in the fifth equality. 
The last lemma and 4.2 imply that

$$
\begin{aligned}
\operatorname{Cov}_{\mathbb{P}}\left(\mathbf{1}_{x \in \mathcal{V}^{u}}, \mathbf{1}_{y \in \mathcal{V}^{u}}\right) & \sim \frac{2 u}{g(0)^{2}} e^{-2 u / g(0)} g(x-y) \\
& \geq c_{u}|x-y|^{2-d}, \quad \text { as }|x-y| \rightarrow \infty .
\end{aligned}
$$

Long range correlations are thus present in the random set $\mathcal{V}^{u}$.

As another consequence of (4.3) and the sub-additivity of the capacity,

$$
\operatorname{cap}\left(\mathbb{A} \cup \mathbb{A}^{\prime}\right) \leq \operatorname{cap} \mathbb{A}+\operatorname{cap} \mathbb{A}^{\prime}
$$

see [27. Proposition 2.2.1(b)], we obtain that

$$
\mathbb{P}\left[\mathbb{A} \cup \mathbb{A}^{\prime} \subset \mathcal{V}^{u}\right] \geq \mathbb{P}\left[\mathbb{A} \subset \mathcal{V}^{u}\right] \mathbb{P}\left[\mathbb{A}^{\prime} \subset \mathcal{V}^{u}\right], \quad \text { for } \mathbb{A}, \mathbb{A}^{\prime} \subset \subset \mathbb{Z}^{d}, u \geq 0,
$$

that is the events $\mathbb{A} \subset \mathcal{V}^{u}$ and $\mathbb{A}^{\prime} \subset \mathcal{V}^{u}$ are positively correlated.

The inequality 4.14) is the special case for the FKG inequality for the measure $Q^{u}$ (see $(3.31)$ ) which was proved in [50]. We present it here for the sake of completeness without proof.

Theorem 4.3 (FKG inequality for random interlacements). Let $A, B \in \mathcal{Y}$ be two increasing events. Then

$$
Q^{u}[A \cap B] \geq Q^{u}[A] Q^{u}[B]
$$

The measure $Q^{u}$ thus satisfies one of the principal inequalities that hold for the Bernoulli percolation. Many of the difficulties appearing when studying random interlacements come from the fact that another important inequality of Bernoulli percolation (the so-called van den Berg-Kesten) does not hold for $Q^{u}$ as one can easily verify by 4.12 .

\subsection{Translation invariance and ergodicity}

We now explore the behavior of random interlacements under translations in $\mathbb{Z}^{d}$. For $x \in \mathbb{Z}^{d}$ and $w \in W$ we define $w+x \in W$ by $(w+x)(n)=$ $w(n)+x, n \in \mathbb{Z}$. For $w \in W^{\star}$, we then set $w^{\star}+x=\pi^{\star}(w+x)$ for $\pi^{\star}(w)=w^{\star}$. Finally, for $\omega=\sum_{i \geq 0} \delta_{\left(w_{i}^{\star}, u_{i}\right)} \in \Omega$ we define

$$
\tau_{x} \omega=\sum_{i \geq 0} \delta_{\left(w_{i}^{\star}-x, u_{i}\right)} .
$$

We let $t_{x}, x \in \mathbb{Z}^{d}$, stand for the canonical shifts of $\{0,1\} \mathbb{Z}^{d}$.

\section{Proposition 4.4.}

(i) $\nu$ is invariant under translations $\tau_{x}$ of $W^{\star}$ for any $x \in \mathbb{Z}^{d}$. 
(ii) $\mathbb{P}$ is invariant under translation $\tau_{x}$ of $\Omega$ for any $x \in \mathbb{Z}^{d}$.

(iii) For any $u \geq 0$, the translation maps $\left(t_{x}\right)_{x \in \mathbb{Z}^{d}}$ define a measure preserving ergodic flow on $\left(\{0,1\} \mathbb{Z}^{d}, \mathcal{Y}, Q^{u}\right)$.

Proof. The proofs of parts (i), (ii) and of the fact that $\left(t_{x}\right)_{x \in \mathbb{Z}^{d}}$ is a measure preserving flow are left as an exercise. They can be found in [47, (1.28) and Theorem 2.1]. We will only show the ergodicity, as its proof is instructive.

Knowing that $\left(t_{x}\right)$ is a measure preserving flow, to prove the ergodicity we only need to show that it is mixing, that is for any $\mathbb{A} \subset \subset \mathbb{Z}^{d}$ and for any $[0,1]$-valued $\sigma\left(Y_{x}: x \in \mathbb{A}\right)$-measurable function $f$ on $\{0,1\}^{\mathbb{Z}^{d}}$, one has

$$
\lim _{|x| \rightarrow \infty} E^{Q^{u}}\left[f \cdot f \circ t_{x}\right]=E^{Q^{u}}[f]^{2} .
$$

In view of $3.35,4$, 4.17 will follow once we show that for any $\mathbb{A} \subset \subset \mathbb{Z}^{d}$ and any $[0,1]$-valued measurable function $F$ on the set of finite point measures on $W_{+}$endowed with the canonical $\sigma$-field,

$$
\lim _{|x| \rightarrow \infty} \mathbb{E}\left[F\left(\mu_{\mathbb{A}, u}\right) F\left(\mu_{\mathbb{A}, u}\right) \circ \tau_{x}\right]=\mathbb{E}\left[F\left(\mu_{\mathbb{A}, u}\right)\right]^{2} .
$$

Since, due to definition of $\tau_{x}$ and $\mu_{\mathbb{A}, u}$, there exists a function $G$ with similar properties as $F$, such that $F\left(\mu_{\mathbb{A}, u}\right) \circ \tau_{x}=G\left(\mu_{\mathbb{A}+x, u}\right), 4.18$ follows from the next lemma.

Lemma 4.5. Let $u \geq 0$ and $\mathbb{A}_{1}$ and $\mathbb{A}_{2}$ be finite disjoint subsets of $\mathbb{Z}^{d}$. Let $F_{1}$ and $F_{2}$ be [0,1]-valued measurable functions on the set of finite point-measures on $W_{+}$endowed with its canonical $\sigma$-field. Then

$$
\begin{aligned}
\left|\mathbb{E}\left[F_{1}\left(\mu_{\mathbb{A}_{1}, u}\right) F_{2}\left(\mu_{\mathbb{A}_{2}, u}\right)\right]-\mathbb{E}\left[F_{1}\left(\mu_{\mathbb{A}_{1}, u}\right)\right] \mathbb{E}\left[F_{2}\left(\mu_{\mathbb{A}_{2}, u}\right)\right]\right| \\
\leq 4 u \operatorname{cap}\left(\mathbb{A}_{1}\right) \operatorname{cap}\left(\mathbb{A}_{2}\right) \sup _{x \in \mathbb{A}_{1}, y \in \mathbb{A}_{2}} g(x-y) .
\end{aligned}
$$

Proof. We write $\mathbb{A}=\mathbb{A}_{1} \cup \mathbb{A}_{2}$ and decompose the Poisson point process $\mu_{\mathbb{A}, u}$ into four point processes on $\left(W_{+}, \mathcal{W}_{+}\right)$as follows:

$$
\mu_{\mathbb{A}, u}=\mu_{1,1}+\mu_{1,2}+\mu_{2,1}+\mu_{2,2},
$$

where

$$
\begin{aligned}
& \mu_{1,1}(\mathrm{~d} w)=1\left\{X_{0} \in \mathbb{A}_{1}, H_{\mathbb{A}_{2}}=\infty\right\} \mu_{\mathbb{A}, u}(\mathrm{~d} w), \\
& \mu_{1,2}(\mathrm{~d} w)=1\left\{X_{0} \in \mathbb{A}_{1}, H_{\mathbb{A}_{2}}<\infty\right\} \mu_{\mathbb{A}, u}(\mathrm{~d} w), \\
& \mu_{2,1}(\mathrm{~d} w)=1\left\{X_{0} \in \mathbb{A}_{2}, H_{\mathbb{A}_{1}}<\infty\right\} \mu_{\mathbb{A}, u}(\mathrm{~d} w), \\
& \mu_{2,2}(\mathrm{~d} w)=1\left\{X_{0} \in \mathbb{A}_{2}, H_{\mathbb{A}_{1}}=\infty\right\} \mu_{\mathbb{A}, u}(\mathrm{~d} w), .
\end{aligned}
$$

In words, the support of $\mu_{1,1}$ are trajectories in the support of $\mu_{\mathbb{A}, u}$ which enter $\mathbb{A}_{1}$ but not $\mathbb{A}_{2}$, the support $\mu_{1,2}$ are trajectories that enter first $\mathbb{A}_{1}$ and then $\mathbb{A}_{2}$, and similarly $\mu_{2,1}, \mu_{2,2}$. 
The $\mu_{i, j}$ 's are independent Poisson point processes, since they are supported on disjoint sets (recall that $\mathbb{A}_{1}$ and $\mathbb{A}_{2}$ are disjoint). Their corresponding intensity measures are given by

$$
\begin{aligned}
& u 1\left\{X_{0} \in \mathbb{A}_{1}, H_{\mathbb{A}_{2}}=\infty\right\} P_{e_{\mathbb{A}}}, \\
& u 1\left\{X_{0} \in \mathbb{A}_{1}, H_{\mathbb{A}_{2}}<\infty\right\} P_{e_{\mathbb{A}}}, \\
& u 1\left\{X_{0} \in \mathbb{A}_{2}, H_{\mathbb{A}_{1}}<\infty\right\} P_{e_{\mathbb{A}}}, \\
& u 1\left\{X_{0} \in \mathbb{A}_{2}, H_{\mathbb{A}_{1}}=\infty\right\} P_{e_{\mathbb{A}}} .
\end{aligned}
$$

We observe that $\mu_{\mathbb{A}_{1}, u}-\mu_{1,1}-\mu_{1,2}$ is determined by $\mu_{2,1}$ and therefore independent of $\mu_{1,1}, \mu_{2,2}$ and $\mu_{1,2}$. In the same way, $\mu_{\mathbb{A}_{2}, u}-\mu_{2,2}-\mu_{2,1}$ is independent of $\mu_{2,2}, \mu_{2,1}$ and $\mu_{1,1}$. We can therefore introduce auxiliary Poisson processes $\mu_{2,1}^{\prime}$ and $\mu_{1,2}^{\prime}$ having the same law as $\mu_{\mathbb{A}_{1}, u}-\mu_{1,1}-\mu_{1,2}$ and $\mu_{\mathbb{A}_{2}, u}-\mu_{2,2}-\mu_{2,1}$ respectively, and satisfying $\mu_{2,1}^{\prime}, \mu_{1,2}^{\prime}, \mu_{i, j}, 1 \leq$ $i, j \leq 2$ are independent. Then

$$
\begin{aligned}
\mathbb{E}\left[F_{1}\left(\mu_{\mathbb{A}_{1}, u}\right)\right] & =\mathbb{E}\left[F_{1}\left(\left(\mu_{\mathbb{A}_{1}, u}-\mu_{1,1}-\mu_{1,2}\right)+\mu_{1,1}+\mu_{1,2}\right)\right] \\
& =\mathbb{E}\left[F_{1}\left(\mu_{2,1}^{\prime}+\mu_{1,1}+\mu_{1,2}\right)\right],
\end{aligned}
$$

and in the same way

$$
\mathbb{E}\left[F_{2}\left(\mu_{\mathbb{A}_{2}}\right)\right]=\mathbb{E}\left[F_{2}\left(\mu_{1,2}^{\prime}+\mu_{2,2}+\mu_{2,1}\right)\right] .
$$

Using 4.23), 4.24 and the independence of the Poisson processes $\mu_{2,1}^{\prime}+$ $\mu_{1,1}+\mu_{1,2}$ and $\mu_{1,2}^{\prime}+\mu_{2,2}+\mu_{2,1}$ we get

$$
\begin{aligned}
& \mathbb{E}\left[F_{1}\left(\mu_{\mathbb{A}_{1}}\right)\right] \mathbb{E}\left[F_{2}\left(\mu_{\mathbb{A}_{2}}\right)\right] \\
& \quad=\mathbb{E}\left[F_{1}\left(\mu_{2,1}^{\prime}+\mu_{1,1}+\mu_{1,2}\right) F_{2}\left(\mu_{1,2}^{\prime}+\mu_{2,2}+\mu_{2,1}\right)\right] .
\end{aligned}
$$

From 4.25 we see that

$$
\begin{aligned}
& \left|\mathbb{E}\left[F_{1}\left(\mu_{\mathbb{A}_{1}}\right) F_{2}\left(\mu_{\mathbb{A}_{2}}\right)\right]-\mathbb{E}\left[F_{1}\left(\mu_{\mathbb{A}_{1}}\right)\right] \mathbb{E}\left[F_{2}\left(\mu_{\mathbb{A}_{2}}\right)\right]\right| \\
& \quad \leq P\left[\mu_{2,1}^{\prime} \neq 0 \text { or } \mu_{1,2}^{\prime} \neq 0 \text { or } \mu_{2,1} \neq 0 \text { or } \mu_{1,2} \neq 0\right] \\
& \quad \leq 2\left(\mathbb{P}\left[\mu_{2,1} \neq 0\right]+\mathbb{P}\left[\mu_{1,2} \neq 0\right]\right) \\
& \quad \leq 2 u\left(P_{e_{\mathbb{A}}}\left[X_{0} \in \mathbb{A}_{1}, H_{\mathbb{A}_{2}}<\infty\right]+P_{e_{\mathbb{A}}}\left[X_{0} \in \mathbb{A}_{2}, H_{\mathbb{A}_{1}}<\infty\right]\right) .
\end{aligned}
$$

We now bound the last two terms in the above equation

$$
\begin{aligned}
P_{e_{\mathbb{A}_{1} \cup \mathbb{A}_{2}}}\left[X_{0} \in \mathbb{A}_{1}, H_{\mathbb{A}_{2}}<\infty\right] & \leq \sum_{x \in \mathbb{A}_{1}} e_{\mathbb{A}_{1}}(x) P_{x}\left[H_{\mathbb{A}_{2}}<\infty\right] \\
& =\sum_{x \in \mathbb{A}_{1}, y \in \mathbb{A}_{2}} e_{\mathbb{A}_{1}}(x) g(x, y) e_{\mathbb{A}_{2}}(y) \\
& \leq \operatorname{cap}\left(\mathbb{A}_{1}\right) \operatorname{cap}\left(\mathbb{A}_{2}\right) \sup _{x \in \mathbb{A}_{1}, y \in \mathbb{A}_{2}} g(x, y) .
\end{aligned}
$$

A similar estimate holds for $P_{e_{\mathbb{A}_{1} \cup \mathbb{A}_{2}}}\left[X_{0} \in \mathbb{A}_{2}, H_{\mathbb{A}_{1}}<\infty\right]$ and the lemma follows. 
As 4.18 follows easily from Lemma 4.5, the proof of Proposition 4.4 is completed.

Proposition 4.4 (iii) has the following standard corollary.

Corollary 4.6 (zero-one law). Let $A \in \mathcal{Y}$ be invariant under the flow $\left(t_{x}: x \in \mathbb{Z}^{d}\right)$. Then, for any $u \geq 0$,

$$
Q^{u}[A]=0 \text { or } 1 \text {. }
$$

In particular, the event

$\operatorname{Perc}(u):=\left\{\omega \in \Omega: \mathcal{V}^{u}(\omega)\right.$ contains an infinite connected component $\}$,

satisfies for any $u \geq 0$

$$
\mathbb{P}[\operatorname{Perc}(u)]=0 \text { or } 1 .
$$

Proof. The first statement follows from the ergodicity by usual techniques. The second statement follows from

$$
\mathbb{P}[\operatorname{Perc}(u)]=Q^{u}\left[\left\{y \in\{0,1\}^{\mathbb{Z}^{d}}: \begin{array}{l}
y \text { contains an infinite } \\
\text { connected component of 1's }
\end{array}\right\}\right]
$$

and the fact that the event on the right-hand side is in $\mathcal{Y}$ and $t_{x}$ invariant.

We now let

$$
\eta(u)=\mathbb{P}\left[0 \text { belongs to an infinite connected component of } \mathcal{V}^{u}\right],
$$

it follows by standard arguments that

$$
\eta(u)>0 \Longleftrightarrow \mathbb{P}[\operatorname{Perc}(u)]=1
$$

In particular defining

$$
u_{\star}=\sup \{u \geq 0: \eta(u)>0\}
$$

we see than the random interlacements model exhibits a phase transition at $u=u_{\star}$. The non-trivial issue is of course to deduce that $0<u_{\star}<\infty$ which we will (partially) do in the next chapter.

\subsection{Comparison with Bernoulli percolation}

We find useful to to draw a parallel between random interlacements and the usual Bernoulli percolation on $\mathbb{Z}^{d}$.

We recall the definition of Bernoulli percolation. Given $p \in[0,1]$, consider on the space $\{0,1\}^{\mathbb{Z}^{d}}$ the probability measure $R^{p}$ under which the 
canonical coordinates $\left(Y_{x}\right)_{x \in \mathbb{Z}^{d}}$ are a collection of i.i.d. Bernoulli $(p)$ random variables. We say that a given site $x$ is open if $Y_{x}=1$, otherwise we say that it is closed. Bernoulli percolation on $\mathbb{Z}^{d}$ is rather well understood, see e.g. monographs [20, 8].

In analogy to 4.33 ) and (4.34), one defines for Bernoulli percolation the following quantities:

$$
\begin{aligned}
\theta(p) & =R^{p} \text { [the origin is connected to infinity by an open path], } \\
p_{c} & =\inf \{p \in[0,1] \text { such that } \theta(p)>0\} .
\end{aligned}
$$

An well-known fact about Bernoulli percolation is that for $d \geq 2, p_{c} \in$ $(0,1)$, see for instance [20, Theorem (1.10)]. In other words, this means that the model undergoes a non-trivial phase transition. As we said, we would like to prove an analogous result for random interlacements percolation, that is to show that $u_{*} \in(0, \infty)$.

Before doing this, let us understand how the random configuration in $\{0,1\}^{\mathbb{Z}^{d}}$ obtained under the measures $R^{p}$ and $Q^{u}$ defined in (3.31) compare to one another.

The first important observation is that under the measure $R^{p}$ every configuration inside a finite set $\mathbb{A}$ has positive probability, while this is not the case with $Q^{u}$. This follows from the following easy claim, which is the consequence of the definitions (3.13), (3.31) of $\Omega$ and $Q^{u}$.

for every $u \geq 0$, almost surely under the measure $Q^{u}$, the set $\left\{x \in \mathbb{Z}^{d}: Y_{x}=0\right\}$ has no finite connected components.

One particular consequence of this fact is that the random interlacements measure $Q^{u}$ will not satisfy the so-called finite energy property. We say that a measure $R$ on $\{0,1\}^{\mathbb{Z}^{d}}$ satisfies the finite energy property if

$$
0<R\left(Y_{y}=1 \mid Y_{z}, z \neq y\right)<1, R \text {-a.s., for all } y \in \mathbb{Z}^{d},
$$

for more details, see 21] (Section 12). Intuitively speaking, this says that not all configurations on a finite set have positive probability under the measure $Q^{u}$. As a consequence, some percolation techniques, such as Burton and Keane's uniqueness argument, will not be directly applicable to $Q^{u}$.

Another important technique in Bernoulli independent percolation is the so-called Peierls-type argument. This argument makes use of the so-called $\star$-paths defined as follows. We say that a sequence $x_{0}, x_{1}, \ldots, x_{n}$ is a $\star$-path if the supremum norm $\left|x_{i}-x_{i+1}\right|_{\infty}$ equals one for every $i=0, \ldots, n-1$. The Peierls argument strongly relies on the fact that, for $p$ sufficiently close to one,

the probability that there is some $\star$-path of 0 's (closed sites) from the origin to $B(0,2 N)$ decays exponentially with $N$. 
This can be used for instance to show that for such values of $p$ there is a positive probability that the origin belongs to an infinite connected component of 1's (open sites).

This type of argument fails in the case of random interlacements. Actually, using (4.4) together with (4.37) we obtain that

for every $u>0$, with positive probability there is an infinite

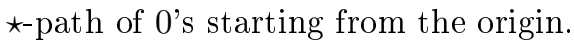

It is actually possible to show that the probability to find a long planar *-path decays, see Chapter 5. However, this is done using a different technique than in Peierls argument.

We further show that random interlacements cannot be compared to Bernoulli percolation via stochastic domination techniques. (This is not completely true if one only considers subspaces of sufficiently large codimension, see Appendix in [12.)

For two measures $Q$ and $Q^{\prime}$ on $\{0,1\}^{\mathbb{Z}^{d}}$, we say that $Q$ dominates $Q^{\prime}$ if

$$
\int f \mathrm{~d} Q \geq \int f \mathrm{~d} Q^{\prime}, \text { for every increasing } f:\{0,1\}^{\mathbb{Z}^{d}} \rightarrow \mathbb{R}_{+} .
$$

Lemma 4.7. For any values of $p \in(0,1)$ and $u>0$, the measure $Q^{u}$ neither dominates nor is dominated by $R^{p}$.

Proof. We start by showing that $Q^{u}$ is not dominated $R^{p}$. For this, let $\mathbb{A}=\mathbb{A}_{L}=[1, L]^{d} \cap \mathbb{Z}^{d}$, and consider the function $f=\mathbf{1}\left\{Y_{x}=\right.$ 1 for every $x \in \mathbb{A}\}$. This function is clearly monotone increasing and for every choice of $p$,

$$
\int f \mathrm{~d} R^{p}=p^{L^{d}}
$$

while for every $u>0$, by 4.3 ,

$$
\int f \mathrm{~d} Q^{u}=\exp \{-u \operatorname{cap} \mathbb{A}\}
$$

Using the inequality (see by (2.16) of [27])

$$
c L^{d-2} \leq \operatorname{cap} \mathbb{A}_{L} \leq c^{\prime} L^{d-2},
$$

it follows that the right-hand side of 4.42 is at most $\exp \left\{-c u L^{d-2}\right\}$. From these considerations, it is clear that for any $u>0$ and any $p \in(0,1)$ we have $\int f \mathrm{~d} R^{p}<\int f \mathrm{~d} Q^{u}$ for some $L$ large enough. This finishes the proof that $R^{p}$ does not dominate $Q^{u}$.

Let us now turn to the proof that $R^{p}$ is not dominated by $Q^{u}$. For this, we consider the function $g=\mathbf{1}\left\{Y_{x}=1\right.$ for some $\left.x \in \mathbb{A}\right\}$, which is clearly increasing and satisfies

$$
\int g \mathrm{~d} R^{p}=1-(1-p)^{L^{d}}
$$


In order to estimate the integral of $g$ with respect to $Q^{u}$, we observe that if the whole cube $\mathbb{A}$ is covered by the random interlacements, then $g=0$. Therefore,

$$
\int g \mathrm{~d} Q^{u} \leq 1-\mathbb{P}\left[\mathbb{A} \subset \mathcal{I}^{u}\right] \stackrel{[3.36}{-} 1-\mathbb{P}_{\mathbb{A}}^{u}\left[\mathbb{A} \subset \bigcup_{w_{+, i} \in \operatorname{supp}\left(\omega_{+}\right)} \operatorname{Range}\left(w_{+, i}\right)\right]
$$

In order to evaluate the above probability, let us first condition on the number of points in the support of $\omega_{+}$.

$$
\begin{aligned}
\mathbb{P}_{\mathbb{A}}^{u}[\mathbb{A} \subset & \left.\bigcup_{w_{+, i} \in \operatorname{supp}\left(\omega_{+}\right)} \operatorname{Range}\left(w_{+, i}\right)\right] \\
\geq & \mathbb{P}_{\mathbb{A}}^{u}\left[\omega_{+}\left(W_{+}\right)=\left\lfloor L^{d-2} \log ^{2} L\right\rfloor\right] \\
& \times P_{e_{\mathbb{A}} / \operatorname{cap}(A)}^{\otimes\left\lfloor L^{d-2} \log ^{2} L\right\rfloor}\left[\mathbb{A} \subset \bigcup_{i=1}^{\left\lfloor L^{d-2} \log ^{2} L\right\rfloor} \operatorname{Range}\left(X^{i}\right)\right]
\end{aligned}
$$

where the last probability is the independent product of $\left\lfloor L^{d-2} \log ^{2} L\right\rfloor$ simple random walks $X^{i}$ s, starting with distribution $e_{\mathbb{A}} / \operatorname{cap}(A)$.

Let us first evaluate the first term in 4.46 , corresponding to the Poisson distribution of $\omega_{+}\left(W_{+}\right)$. For this, we write $\alpha=u \operatorname{cap}(\mathbb{A})$ and $\beta=$ $\left\lfloor L^{d-2} \log ^{2} L\right\rfloor$. Then, using de Moivre-Stirling's approximation, we obtain that the left term in the above equation is

$$
\frac{e^{-\alpha} \alpha^{\beta}}{\beta !} \geq c \frac{e^{-\alpha+\beta}}{\sqrt{\beta}}\left(\frac{\alpha}{\beta}\right)^{\beta}
$$

and using again 4.43, for $L \geq c(u)$ sufficiently large,

$$
\begin{aligned}
& \geq \exp \left\{-c_{u} L^{d-2}+\left\lfloor L^{d-2} \log ^{2} L\right\rfloor\right\}\left(\frac{c_{u}}{\log ^{2} L}\right)^{\beta} \\
& \geq\left(\frac{c_{u}}{\log ^{2} L}\right)^{\beta} \geq \exp \left\{-c_{u} \log \left(\log ^{2} L\right) \cdot\left(\log ^{2} L\right) L^{d-2}\right\} \\
& \geq \exp \left\{-c_{u}\left(\log ^{3} L\right) L^{d-2}\right\} .
\end{aligned}
$$

To bound the second term in 4.46 , fix first some $z \in \mathbb{A}$ and estimate

$$
\begin{aligned}
P_{e_{\mathbb{A}} / \operatorname{cap}(A)}^{\otimes \beta} & {\left[z \in \cup_{i=1}^{\beta} \operatorname{Range}\left(X_{i}\right)\right] } \\
& =1-\left(P_{e_{\mathbb{A}} / \operatorname{cap}(A)}\left[z \notin \operatorname{Range}\left(X_{1}\right)\right]\right)^{\beta} \\
& \quad \geq 1-\left(1-c L^{2-d}\right)^{c L^{d-2} \log ^{2} L} \geq 1-e^{-c \log ^{2} L} .
\end{aligned}
$$


Therefore, by a simple union bound, we obtain that the right-hand side of 4.46 is bounded from below by $1 / 2$ as soon as $L$ is large enough depending on $u$. Putting this fact together with $(4.46)$ and $(4.48)$, we obtain that

$$
\int g \mathrm{~d} Q^{u} \leq 1-c \exp \left\{-c_{u}\left(\log ^{3} L\right) L^{d-2}\right\}
$$

which is smaller than the right hand side of (4.44) for $L$ large enough depending on $p$ and $u$. This proves that $Q^{u}$ does not dominate $R^{p}$ for any values of $p \in(0,1)$ or $u>0$, finishing the proof of the lemma.

\subsection{Notes}

Various interesting properties of random interlacements have been established in recent works. Let us mention here a few of them.

In Theorem 2.4 of [47] and in Appendix A of [12], the authors provide a comparison between Bernoulli percolation and random interlacements on sub-spaces (slabs) of $\mathbb{Z}^{d}$. If the co-dimension of the subspace in question is at least three, then the domination by Bernoulli percolation holds. This domination was useful in simplifying arguments for the non-triviality of the phase transition for the vacant set and the behavior of the chemical distance in $\mathcal{I}^{u}$ in high dimensions, see Remark 2.5 3 of [47] and Appendix A of 12 for more details.

Another important result on the domination of random interlacements says that the component of the interlacement set $\mathcal{I}^{u}$ containing a given point is dominated by the range of a certain branching random walk, see Propositions 4.1 and 5.1 in $[52$. This result does not add much for the study of random interlacements on $\mathbb{Z}^{d}$ (as the mentioned branching random walk covers the whole lattice almost surely), but it has been valuable for establishing results for random interlacements on non-amenable graphs, see 52 .

It may be interesting to observe that the measure $Q^{u}$ does not induce a Markov field. More precisely, this means that the law of $\mathcal{V}^{u} \cap A$ conditioned on $\mathcal{V}^{u} \cap A^{c}$ is not the same as that of $\mathcal{V}^{u} \cap A$ conditioned on $\mathcal{V}^{u} \cap\{x \in$ $A^{c} ; x \sim A$ \}. See [50], Remark 3.33 ) for more details.

After defining random interlacements on different classes of graphs (see [50] for such a construction), one could get interested in understanding for which classes of graphs there exists a non-trivial phase transition at some critical $0<u_{\star}<\infty$. This question is expected to be very relevant to the study of the disconnection time in arbitrary cylinders, see 43 , and [57. Some results in this direction have been established already when the graph in question is a tree, see Theorem 5.1 of [50], or under some hypotheses on the isoperimetrical profile of the graph, see Theorems 4.1 and 4.2 of [50] and Proposition 8.1 of [52]. 


\section{Chapter 5}

\section{Renormalization}

In this chapter we are going to prove that $u_{\star}>0$ for $d$ sufficiently large ( $d \geq 7$ is enough). This only establishes one side of the non-triviality of $u_{\star}$, but illustrates the multi-scale renormalization, which is employed in several other problems of dependent percolation and particle systems. The biggest advantage of the renormalization scheme is that it does not enter too much on the kind of dependence involved in the problem. Roughly speaking, having only a control on the decay of dependence (such as in Lemma 4.5 we may have enough to obtain global statements about the measure under consideration.

\subsection{Renormalization scheme}

To better control the dependences using Lemma 4.5 we need to understand the decay of the Green's function for the simple random walk on $\mathbb{Z}^{d}$. We quote from Theorem 1.5.4 of [27] that

$$
g(x) \leq c|x|^{2-d} .
$$

The main result concerning the non-triviality of the critical value is

Theorem 5.1. For any $d \geq 3$, we have that $0<u_{\star}<\infty$.

which was proved in [45] and 40].

What we intend to prove in this chapter is a part of the above, more precisely,

Theorem 5.2. For $d \geq 7$, we have that $u_{\star}>0$.

Proof. The proof we present here follows the arguments of Proposition 4.1 in [47] with some minor modifications. 
We will use this bound in the renormalization argument we mentioned above. This renormalization will take place on $\mathbb{Z}^{2} \subset \mathbb{Z}^{d}$, which is identified by the isometry $\left(x_{1}, x_{2}\right) \mapsto\left(x_{1}, x_{2}, 0, \ldots, 0\right)$. Throughout the text we make no distinction between $\mathbb{Z}^{2}$ and its isometric copy inside $\mathbb{Z}^{d}$.

We say that $\tau:\{0, \cdots, n\} \rightarrow \mathbb{Z}^{2}$ is a $\star$-path if

$$
|\tau(k+1)-\tau(k)|_{\infty}=1 \text {, for all } k \in\{0, \cdots, n-1\},
$$

where $|p|_{\infty}$ is the maximum of the absolute value of the two coordinates of $p \in \mathbb{Z}^{2}$. Roughly speaking, the strategy of the proof is to prove that with positive probability there is no $\star$-path in $\mathcal{I}^{u} \cap \mathbb{Z}^{2}$ surrounding the origin. This will imply by a duality argument that there exists an infinite connected component in $\mathcal{V}^{u}$.

We now define a sequence of non-negative integers which will represent the scales involved in the renormalization procedure. For any $L_{0} \geq 2$, let

$$
\begin{gathered}
L_{n+1}=l_{n} L_{n}, \text { for every } n \geq 0, \\
\text { where } l_{n}=100\left\lfloor L_{n}^{a}\right\rfloor \text { and } a=\frac{1}{1000} .
\end{gathered}
$$

Here $\lfloor a\rfloor$ represent the largest integer smaller or equal to $a$.

In what follows, we will consider a sequence of boxes in $\mathbb{Z}^{2}$ of size $L_{n}$, but before, let us consider the set of indices

$$
J_{n}=\{n\} \times \mathbb{Z}^{2}, \text { for } n \geq 0 .
$$

For $m=(n, q) \in J_{n}$, we consider the box

$$
D_{m}=\left(L_{n} q+\left[0, L_{n}\right)^{2}\right) \cap \mathbb{Z}^{2},
$$

And also

$$
\widetilde{D}_{m}=\bigcup_{i, j \in\{-1,0,1\}} D_{(n, q+(i, j))} .
$$

As we mentioned, our strategy is to prove that the probability of finding a $\star$-path in the set $\mathcal{I}^{u} \cap \mathbb{Z}^{2}$ that separates the origin from infinity in $\mathbb{Z}^{2}$ is smaller than one. We do this by bounding the probabilities of the following crossing events

$$
B_{m}^{u}=\left\{\begin{array}{c}
\omega \in \Omega: \text { there exists a } \star \text {-path in } \mathcal{I}^{u} \cap \mathbb{Z}^{2} \\
\text { connecting } D_{m} \text { to the complement of } \widetilde{D}_{m}
\end{array}\right\},
$$

where $m \in J_{n}$. For $u>0$, we write

$$
q_{n}^{u}=\mathbb{P}\left[B_{(n, 0)}^{u} \stackrel{\text { Proposition } 4.4}{=} \sup _{m \in J_{n}} \mathbb{P}\left[B_{m}^{u}\right] .\right.
$$

In order to show that for $u$ small enough $q_{n}^{u}$ decays with $n$, we are going to obtain an induction relation between $q_{n}^{u}$ and $q_{n+1}^{u}$ (that were defined in 


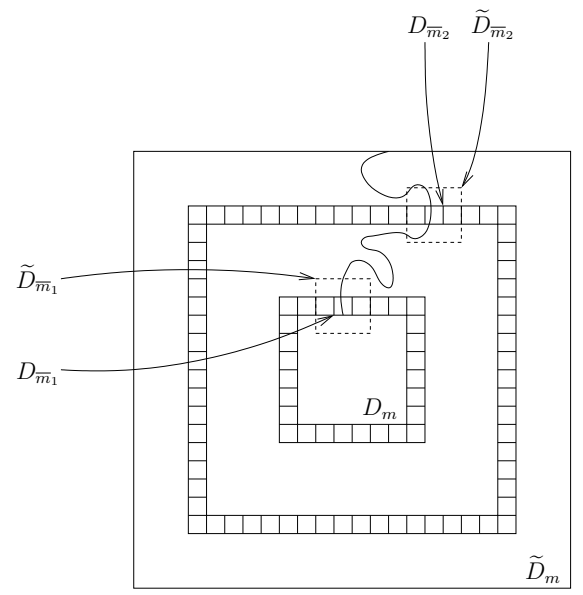

Figure 5.1: The figure shows all the boxes with indices in $\mathcal{K}_{1}$ and $\mathcal{K}_{2}$. Note that the event $B_{m}^{u}$ implies $B_{\bar{m}_{1}}^{u}$ and $B_{\bar{m}_{2}}^{u}$ for some $\bar{m}_{1} \in \mathcal{K}_{1}$ and $\bar{m}_{2} \in \mathcal{K}_{2}$.

terms of two different scales). For this we consider, for a fixed $m \in J_{n+1}$, the indices of boxes in the scale $n$ that are in the "boundary of $D_{m}$ ". More precisely

$$
\mathcal{K}_{1}^{m}=\left\{\bar{m}_{1} \in J_{n}: D_{\bar{m}_{1}} \subset D_{m} \text { and } D_{\bar{m}_{1}} \text { is neighbor of } \mathbb{Z}^{2} \backslash D_{m}\right\} .
$$

And the indices of boxes at the scale $n$, having a point at distance $L_{n+1} / 2$ from $D_{m}$, i.e.

$$
\mathcal{K}_{2}^{m}=\left\{\bar{m}_{2} \in J_{n}: D_{\bar{m}_{2}} \cap\left\{x \in \mathbb{Z}^{2}: d_{\mathbb{Z}^{2}}\left(x, D_{m}\right)=L_{n+1} / 2\right\} \neq \emptyset\right\} .
$$

The boxes associated with the two sets of indices above are shown in Figure 5.1. In this figure we also illustrate that the event $B_{m}^{u}$ implies the occurrence of both $B \frac{u}{m_{1}}$ and $B_{\bar{m}_{2}}^{u}$ for some choice of $\bar{m}_{1} \in \mathcal{K}_{1}^{m}$ and $\bar{m}_{2} \in \mathcal{K}_{2}^{m}$.

This, with a rough counting argument, allows us to conclude that

$$
q_{m}^{u} \leq c l_{n}^{2} \sup _{\substack{\bar{m}_{1} \in \mathcal{K}_{1}^{m} \\ \bar{m}_{2} \in \mathcal{K}_{2}^{m}}} \mathbb{P}\left[B_{\bar{m}_{1}}^{u} \cap B_{\bar{m}_{2}}^{u}\right] \text {, for all } u \geq 0 .
$$

We now want to control the dependence of the process in the two boxes 
$\widetilde{D}_{\bar{m}_{1}}$ and $\widetilde{D}_{\bar{m}_{2}}$. For this we will use Lemma 4.5 which provides that

$$
\begin{aligned}
& \mathbb{P}\left[B_{\bar{m}_{1}}^{u} \cap B_{\bar{m}_{1}}^{u}\right] \\
& \quad \leq \mathbb{P}\left[B_{\bar{m}_{1}}^{u}\right] \mathbb{P}\left[B_{\bar{m}_{1}}^{u}\right]+4 u \operatorname{cap}\left(\widetilde{D}_{\bar{m}_{1}}\right) \operatorname{cap}\left(\widetilde{D}_{\bar{m}_{2}}\right) \sup _{x \in \widetilde{D}_{\bar{m}_{1}}, y \in \widetilde{D}_{\bar{m}_{2}}} g(x-y) \\
& \quad \stackrel{5.1 p}{\leq}\left(q_{n}^{u}\right)^{2}+c L_{n}^{2} \frac{L_{n}^{2}}{L_{n+1}^{5}}
\end{aligned}
$$

where we assumed in the last step that $u \leq 1$. Using (5.11) and taking the supremum over $m \in J_{n+1}$, we conclude that

$$
q_{n+1}^{u} \leq c l_{n}^{2}\left(\left(q_{n}^{u}\right)^{2}+L_{n}^{4} L_{n+1}^{-5}\right) .
$$

With help of this recurrence relation, we prove the next lemma, which shows that for some choice of $L_{0}$ and for $u$ taken small enough, $q_{n}^{u}$ goes to zero sufficiently fast with $n$. The recurrence in the next lemma is only possible due to the assumption $d \geq 7$, giving us the decay $L_{n+1}^{-5}$ in the above equation.

Lemma 5.3. There exist $L_{0}$ and $\bar{u}=\bar{u}\left(L_{0}\right)>0$, such that

$$
q_{n}^{u} \leq \frac{\emptyset}{l_{n}^{2} L_{n}^{1 / 2}}
$$

for every $u<\bar{u}$.

Proof of Lemma 5.3. We define the sequence

$$
b_{n}=q_{0} l_{n}^{2} q_{n}^{u}, \text { for } n \geq 0 .
$$

The equation (5.13) can now be rewritten as

$$
b_{n+1} \leq c\left(\left(\frac{l_{n+1}}{l_{n}}\right)^{2} b_{n}^{2}+\left(l_{n+1} l_{n}\right)^{2} L_{n}^{4} L_{n+1}^{-5}\right), \text { for } n \geq 0 .
$$

With 5.3 one concludes that $\left(l_{n+1} l_{n}\right)^{2} \leq c L_{n}^{2 a} L_{n+1}^{2 a} \leq c L_{n}^{4 a+2 a^{2}}$. Inserting this in (5.16) and using again (5.3), we obtain

$$
b_{n+1} \leq q_{\mathbb{1}}\left(L_{n}^{2 a^{2}} b_{n}^{2}+L_{n}^{2 a^{2}-a-1}\right) \leq q_{\mathbb{1}} L_{n}^{2 a^{2}}\left(b_{n}^{2}+L_{n}^{-1}\right) .
$$

We use this to show that, if for some $L_{0}>(2 q 1)^{4}$ and $u \leq 1$ we have $b_{n} \leq$ $L_{n}^{-1 / 2}$, then the same inequality also holds for $n+1$. Indeed, supposing $b_{n} \leq L_{n}^{-1 / 2}$, we have

$$
\begin{aligned}
& b_{n+1} \leq 2 q_{\square} L_{n}^{2 a^{2}-1} \stackrel{\sqrt[5.3]{\leq}}{\leq} 2 q_{\square} L_{n+1}^{-1 / 2} L_{n}^{1 / 2(1+a)+2 a^{2}-1} \\
& \stackrel{5.3}{\leq} 2 q_{\square} L_{n+1}^{-1 / 2} L_{0}^{-1 / 4} \leq L_{n+1}^{-1 / 2} \text {, }
\end{aligned}
$$


which is the statement of the lemma. So all we still have to prove is that $b_{0} \leq L_{0}^{-1 / 2}$ for $L_{0}>(2 q)^{4}$ and small enough $u$. Indeed,

$$
\begin{aligned}
& b_{0} \stackrel{[5.15}{-} q_{0} l_{0}^{2} q_{0}^{u} \leq q_{0}^{2} \sup _{m \in J_{0}} \mathbb{P}\left[\mathcal{I}^{u} \cap \widetilde{D}_{m} \neq \emptyset\right] \\
& \quad \leq q \square L_{0}^{2 a+2} \sup _{x \in V} \mathbb{P}\left[x \in \mathcal{I}^{u}\right] \stackrel{4.3}{\leq} q_{1} L_{0}^{2 a+2}\left(1-e^{-\operatorname{cap}(\{x\}) u}\right) .
\end{aligned}
$$

For some $L_{0}>(2 q 1)^{4}$, we take $u\left(L_{0}\right)$ small enough such that $b_{0} \leq L_{0}^{-1 / 2}$ for any $u \leq u\left(L_{0}\right)$. This concludes the proof of Lemma 5.3

We now use this lemma to show that with positive probability, one can find an infinite connection from $(0,0)$ to infinite in the set $\mathcal{V}^{u} \cap \mathbb{Z}^{d}$. For this we choose $L_{0}$ and $u<u\left(L_{0}\right)$ as in the lemma. Writing $B_{M}$ for the set $[-M, M] \times[-M, M] \subset \mathbb{Z}^{2}$, we have

$1-\eta(u,(0,0)) \leq \mathbb{P}\left[(0,0)\right.$ is not in an infinite component of $\left.\mathcal{V}^{u} \cap \mathbb{Z}^{2}\right]$

$$
\begin{aligned}
& \leq \mathbb{P}\left[\mathcal{I}^{u} \cap B_{M} \neq \emptyset\right]+\mathbb{P}\left[\begin{array}{c}
\text { there is a } \star \text {-path in } \mathbb{Z}^{2} \backslash B_{M} \\
\text { surrounding the point }(0,0) \text { in } \mathbb{Z}^{2}
\end{array}\right] \\
& \leq\left(1-\exp \left(-u \cdot \operatorname{cap}\left(B_{M}\right)\right)\right) \\
& +\sum_{n \geq n_{0}} \mathbb{P}\left[\begin{array}{c}
\mathcal{I}^{u} \cap \mathbb{Z}^{2} \backslash B_{M} \text { contains a } \star \text {-path surrounding }(0,0) \text { and } \\
\text { passing through some point in }\left[L_{n}, L_{n+1}-1\right] \times\{0\} \in \mathbb{Z}^{2}
\end{array}\right]
\end{aligned}
$$

The last sum can be bounded by $\sum_{n \geq n_{0}} \sum_{m} \mathbb{P}\left[B_{m}^{u}\right]$ where the index $m$ runs over all labels of boxes $D_{m}$ at level $n$ that intersect $\left[L_{n}, L_{n+1}-1\right] \times\{0\} \subset$ $\mathbb{Z}^{2}$. Since the number of such $m$ 's is at most $l_{n} \leq c L_{n}^{a}$,

$$
1-\eta(u,(0,0)) \leq c L_{n_{0}}^{2} u+\sum_{n \geq n_{0}} c L_{n}^{a} L_{n}^{-1 / 2} \stackrel{5.3}{\leq} c\left(L_{n_{0}}^{2} u+\sum_{n \geq n_{0}} L_{n}^{-1 / 4}\right) .
$$

Choosing $n_{0}$ large and $u \leq u\left(L_{0}, n_{0}\right)$, we obtain that the percolation probability is positive. So that $u_{\star}>0$ finishing the proof of Theorem 5.2

\section{$5.2 \quad$ Notes}

As we mentioned below Theorem 5.2 its proof is largely inspired by Proposition 4.1 of [47. It illustrates well how the renormalization scheme can help us to deal with the dependence present in the measure $Q^{u}$. We would like to mention two important improvements that were developed over the above techniques. 
Sprinkling is a technique that first appeared for random interlacements in Section 3 of [47]. It allows us to obtain estimates similar to that of Lemma 4.5 but by "sprinkling" some extra trajectories into the picture, one can wash away the dependence even further and obtain better error estimates. This technique has been for instance useful later in [44] and [53].

Another modification of the renormalization we presented here, that has been very efficient in boosting the decay of dependence (such as in (5.3) are the so-called decoupling inequalities. The idea behind this procedure is to create a binary tree, which encodes the occurrence of bad events in various sub-boxes. Then, by carefully comparing the number of such trees with the probability that they are corrupted, one obtains a bound on the probability of bad events. This idea first appeared in [51] and [41, being later generalized in [48.

Renormalization tools very similar to the ones described above have proved themselves useful in obtaining various results, both for random interlacements and for other dependent percolation processes, see for instance [54] and [23].

Remarkably, some more elaborate renormalization schemes were able to establish the non-triviality of the phase transition that $\mathcal{V}^{u}$ undergoes at $u_{\star}$. More precisely, it was shown that

$$
u_{\star}<\infty \text { for any } d \geq 3, \text { see Theorem } 3.5 \text { in [47, }
$$

and

$$
u_{\star}>0 \text { for any } d \geq 3 \text {, see Theorem } 3.4 \text { in [40]. }
$$




\section{Chapter 6}

\section{Interlacement set}

Up to now, we have mainly investigated the vacant set left by a random walk on a finite graph or by random interlacements. In this chapter we are going to consider the interlacement set $\mathcal{I}^{u}$ of the random interlacements, which is of course related to the trace left by random walk on a graph, $\left\{X_{0}, \ldots, X_{u|G|}\right\}$.

Contrary to the Bernoulli percolation, where by changing $p$ to $1-p$, one can interchange the laws of open and closed sites, the interlacement set $\mathcal{I}^{u}$ is a genuine object: It cannot be obtained from $\mathcal{V}^{u}$ by a re-parametrization. Its properties should be thus studied separately.

\subsection{Main properties of random interlacements}

In general, understanding of $\mathcal{I}^{u}$ is easier than of $\mathcal{V}^{u}$. The main reason for this is that the independence coming from the Poisson point process structure is easier to explore when considering the interlacement set; $\mathcal{I}^{u}$ is the trace left by the Poisson point process, not its complement. In addition, there is no phase transition for the connectivity of $\mathcal{I}^{u}$, which further simplifies the studies of $\mathcal{I}^{u}$ :

Theorem 6.1 (47, 12]). For every $d \geq 3$, the interlacement set $\mathcal{I}^{u}$ on $\mathbb{Z}^{d}$ satisfies

$$
\mathbb{P}\left[\mathcal{I}^{u} \text { is connected for all } u>0\right]=1 .
$$

Proof. The proof of the theorem is based on the following proposition, that is much stronger than (6.1). To state it, it is useful to introduce the following notation. We write $f(n)=\mathfrak{s . e} .(n)$ when $f(n) \leq c e^{-c^{\prime} n^{\delta}}$ for some $c, c^{\prime}, \delta \in(0, \infty)$, 's.e.' staying for 'stretched-exponentially' small. We write $f(n)=1-\mathfrak{s e .} .(n)$, when $1-f(n)=\mathfrak{s . e} .(n)$. Observe that $n^{c} \mathfrak{s . e .}(n)=\mathfrak{s . e .}(n)$ for any fixed $c>0$. So, it is quite convenient to use this notation e.g. in 
the following situation: assume that we have at most $n^{c}$ events, each of probability bounded from above by s.e. $(n)$. Then, the probability of their union is $\mathfrak{s . e} .(n)$ as well.

Recall from Chapter 3 that $\mathbb{P}$ denotes the law of the Poisson point process on $W^{\star} \times[0, \infty)$ defining the random interlacements and that $\mu_{\mathbb{A}, u}$ is the map that selects from $\omega$ those trajectories which intersect $\mathbb{A}$ and have labels smaller than $u$. Let us define $\mathcal{I}_{n}^{u}$ to be range of the trajectories intersecting $B(n)$,

$$
\mathcal{I}_{n}^{u}(\omega)=\bigcup_{w \in \operatorname{supp} \mu_{B(n), u}(\omega)} \text { Range } w .
$$

Proposition 6.2. For every $d \geq 3$ and $0<u<u^{\prime}$,

$$
\begin{aligned}
& \mathbb{P}\left[\mathcal{I}_{n}^{u} \text { is connected }\right]=1-\mathfrak{s} \cdot \mathrm{e} .(n) . \\
& \mathbb{P}\left[\forall w \in \operatorname{supp}\left(\mu_{B(n), u^{\prime}}-\mu_{B(n), u}\right) \text { is connected to } \mathcal{I}_{n}^{u}\right]=1-\mathfrak{s . e} .(n) .
\end{aligned}
$$

Let us first explain how to Proposition 6.2 implies Theorem 6.1. Assume for the moment that the claim of Theorem 6.1 is false, that is

$$
\mathbb{P}\left[\text { there exists } u>0 \text { such that } \mathcal{I}^{u} \text { is not connected }\right]>0 \text {. }
$$

Then, one can find $\varepsilon>0$ and $0<u<u^{\prime}<\infty$ such that

$$
\mathbb{P}\left[\text { there exists } \tilde{u} \in\left[u, u^{\prime}\right] \text { such that } \mathcal{I}^{\tilde{u}} \text { is not connected }\right] \geq \varepsilon
$$
and so,

$$
\liminf _{n \rightarrow \infty} \mathbb{P}\left[\text { there exists } \tilde{u} \in\left[u, u^{\prime}\right] \text { such that } \mathcal{I}_{n}^{\tilde{u}} \text { is not connected }\right] \geq \varepsilon \text {. }
$$

This, however, contradicts Proposition 6.2 since, by the second claim of the proposition, the probability that $\mathcal{I}_{n}^{\tilde{u}}$ is connected for all $\tilde{u} \in\left[u, u^{\prime}\right]$ approaches 1 as $n \rightarrow \infty$. So, 6.5 cannot be true.

We now show Proposition 6.2. The proof that we present is more complicated than necessary, but it has some important implications that we explore later. It is a simplified version of some of the arguments used in [36, 38, 12.

The proof is based on the following heuristics. It is known fact that one random walk trajectory is a two-dimensional object. Considering all trajectories in supp $\mu_{B(n), u}$ intersecting this trajectory (including this trajectory), we obtain a four-dimensional connected object. Another iterations of this procedure give us $2 k$-dimensional objects (if $2 k \leq d$ ). The capacity of a $2 k$-dimensional object intersected with a ball of size $n$ is roughly comparable with $n^{2 k}$, as long as $2 k \leq d-2$. Hence, when $2 k \geq d-2$, this $2 k$-dimensional object saturates the $B(n)$ in terms of capacity, and 
any other random walk trajectory has a non-negligible chance to hit it. Iterating then the construction once more, if necessary, gives an object which is very likely hit by every other walk in $\operatorname{supp} \mu_{B(n), u}$, implying that $\mathcal{I}_{n}^{u}$ is connected.

Proof of Proposition 6.2. We start the formal proof. In $d=3$, 4, the proposition is a direct consequence of Theorem 7.6 in 26 which states that two independent random walks in $d=3,4$ intersect (infinitely often) with probability 1 , and of the fact that the number of trajectories in $\operatorname{supp} \mu_{B(n), u}$ is finite $\mathbb{P}$-a.s. Therefore we will assume that $d \geq 5$ in the proof.

Will repeatedly use the following lemma.

Lemma 6.3. Let $\mathbb{A} \subset \mathbb{Z}^{d}$, and $x \in \mathbb{Z}^{d}$ be such that $\max _{y \in \mathbb{A}} \operatorname{dist}(x, y) \leq r$. Then, for some $c>0$ depending on the dimension only

$$
P_{x}\left[H_{\mathbb{A}} \leq r^{2} \wedge H_{B(x, 2 r)^{c}}\right] \geq c r^{2-d} \operatorname{cap} \mathbb{A} \text {. }
$$

Proof. The proof of the lemma is a variant of the proof of (4.8). The only non-trivial new ingredient is the observation that for $x, y$ with $\operatorname{dist}(x, y) \leq$ $r$ the 'truncated Green function' satisfies

$$
g_{B\left(x, 2 r^{2}\right)}\left(x, y ; r^{2}\right):=\sum_{i=0}^{r^{2} \wedge H_{B\left(x, 2 r^{2}\right)^{c}}} P_{x}\left[X_{i}=y\right] \geq c g(x, y) .
$$

We therefore omit the details and leave the proof of $(6.9)$ and of the lemma as exercise.

To apply Lemma 6.3 we need to estimate capacities of various collections of random walk trajectories. The estimate we will use is given in Lemmas 6.5, 6.6 below. We start with a technical estimate.

Lemma 6.4. Let $d \geq 5,\left(x_{k}\right)_{k \geq 1}$ be a sequence in $\mathbb{Z}^{d}$, and let $X^{k}$ be a sequence of independent simple random walks on $\mathbb{Z}^{d}$ with $X_{0}^{k}=x_{k}$. Then for all positive integers $N$ and $n$ and for all $\left(x_{k}\right)_{k \geq 1}$,

$$
E\left[\sum_{k, l=1}^{N} \sum_{i, j=n+1}^{2 n} g\left(X_{i}^{k}, X_{j}^{l}\right)\right] \leq C\left(N n+N^{2} n^{3-d / 2}\right) .
$$

Proof. Let $X$ be a simple random walk with $X_{0}=0$, then for all $y \in \mathbb{Z}^{d}$ and for all positive integers $k$, by the Markov property,

$$
E g\left(X_{k}, y\right)=\sum_{i=k}^{\infty} P\left[X_{i}=y\right] \leq C \sum_{i=k}^{\infty} i^{-d / 2} \leq C k^{1-d / 2}
$$


Here we used the inequality $\sup _{y \in \mathbb{Z}^{d}} P\left[X_{k}=y\right] \leq C k^{-d / 2}$, see [42, Proposition 7.6] In order to prove (6.10), we consider separately the cases $k=l$ and $k \neq l$. In the first case, the Markov property and the fact that $g(x, y)=g(x-y)$ imply

$$
\begin{gathered}
E\left[\sum_{k=1}^{N} \sum_{i, j=n+1}^{2 n} g\left(X_{i}^{k}, X_{j}^{k}\right)\right]=N E\left[\sum_{i, j=n+1}^{2 n} g\left(X_{|i-j|}\right)\right] \\
\frac{6.11}{\leq} C N n\left(1+\sum_{i=1}^{n} i^{1-d / 2}\right) \stackrel{(d \geq 5)}{\leq} C N n .
\end{gathered}
$$

In the case $k \neq l$, an application of 6.11 gives

$$
E\left[\sum_{i, j=n+1}^{2 n} g\left(X_{i}^{k}, X_{j}^{l}\right)\right] \leq n^{2} C n^{1-d / 2} .
$$

This completes the proof.

Let $\left(X^{k}\right)_{k \geq 1}$ be the collection of independent random walks, as in the previous lemma. For $\mathbb{A} \subset \mathbb{Z}^{d}$, let $T_{\mathbb{A}}^{k}=\inf \left\{i \geq 0: X_{i}^{k} \notin \mathbb{A}\right\}$ be the exit time of $X^{k}$ from $\mathbb{A}$. For positive integers $N$ and $n$, define the subset $\Phi(N, n)$ of $\mathbb{Z}^{d}$

$$
\Phi(N, n)=\bigcup_{k=1}^{N}\left\{X_{i}^{k}: 1 \leq i \leq n \wedge T_{B\left(x_{k}, 2 n^{1 / 2}\right)}^{k}\right\} .
$$

Lemma 6.5. For any sequence $\left(x_{i}\right)_{i \geq 1} \subset \mathbb{Z}^{d}$ and for all positive integers $N$ and $n$,

$$
\operatorname{cap} \Phi(N, n) \leq C N n
$$

and

$$
E \operatorname{cap} \Phi(N, n) \geq c \min \left(N n, n^{(d-2) / 2}\right)
$$

Proof. The upper bound follows from the sub-additivity of the capacity 4.13) and the fact that $\operatorname{cap}(\{x\})=g(0,0)^{-1}<\infty$.

We proceed with the lower bound on $E$ cap $\Phi(N, n)$. By Kolmogorov's maximal inequality applied coordinate-wise, for each $\lambda>0$ and $n \geq 1$,

$$
P\left[\max _{1 \leq i \leq n}\left|X_{i}\right| \geq \lambda\right] \leq \frac{n}{\lambda^{2}} .
$$

Let $J$ be the random set $J=\left\{1 \leq k \leq N: \sup _{1<i<n}\left|X_{i}^{k}-x_{k}\right| \leq 2 n^{1 / 2}\right\}$, and $D$ the event $D=\{|J| \geq N / 4\}$. From (6.17), it follows that $E|J| \geq$ $N\left(1-\frac{n}{4 n}\right) \geq \frac{N}{2}$. Since $|J| \leq N$, we get $P[D] \geq \frac{1}{3}$. 
To obtain a lower bound on the capacity, the following variational formula (see Proposition 2.3 [56]) is useful:

$$
\operatorname{cap} \mathbb{A}=(\inf \mathcal{E}(\nu))^{-1}
$$

where the energy $\mathcal{E}(\nu)$ of a measure $\nu$ is given by

$$
\mathcal{E}(\nu)=\sum_{x, y \in \mathbb{Z}^{d}} \nu(x) \nu(y) g(x, y)
$$

and the infimum in $(6.18)$ is taken over all probability measures supported on $\mathbb{A}$.

Taking $\nu$ to be $\nu(x)=\frac{2}{|J| n} \sum_{k \in J} \sum_{i=n / 2}^{n} \mathbf{1}\left\{X_{i}^{k}=x\right\}$, which is obviously supported on $\Phi(N, n)$, we obtain

$$
E \operatorname{cap} \Phi(N, n) \geq E\left[\mathcal{E}(\nu)^{-1}\right] \geq E\left[\mathcal{E}(\nu)^{-1} ; A\right] .
$$

Therefore, in order to prove $(6.16)$, it suffices to show that

$$
E\left[\left(\frac{4}{|J|^{2} n^{2}} \sum_{k, l \in J} \sum_{i, j=n / 2}^{n} g\left(X_{i}^{k}, X_{j}^{l}\right)\right)^{-1} ; A\right] \geq c \min \left(N n, n^{(d-2) / 2}\right) .
$$

Using the Cauchy-Schwarz inequality and the definition of the event $D$, the left-hand side of the last display can be bounded from below by

$$
c N^{2} n^{2} P(D)^{2}\left(E\left[\sum_{k, l \in J} \sum_{i, j=n / 2}^{n} g\left(X_{i}^{k}, X_{j}^{l}\right) ; D\right]\right)^{-1}
$$

Since $J$ is a subset of $\{1, \ldots, N\}$, this is larger than

$$
\begin{aligned}
(N / 4)^{2} n^{2} P(D)^{2}\left(E\left[\sum_{k, l=1}^{N} \sum_{i, j=n / 2}^{n} g\left(X_{i}^{k}, X_{j}^{l}\right)\right]\right)^{-1} \\
\stackrel{c N^{2} n^{2}}{\geq} \frac{c \min \left(N n, n^{(d-2) / 2}\right) .}{N n+N^{2} n^{3-d / 2}} \geq c \min (.)
\end{aligned}
$$

This completes the proof.

Lemma 6.6. In the setting of the previous lemma, assume that for some $\delta>0, n^{\delta} \leq N \leq n^{\frac{d-4}{2}-\delta}$, then for every $\varepsilon>0$ sufficiently small

$$
P\left[\operatorname{cap} \Phi(N, n) \geq(N n)^{1-\varepsilon}\right]=1-\text { s.e. }(n) .
$$

Proof. To see that this lemma holds it is sufficient to split the trajectories into $n^{\varepsilon}$ pieces of length $n^{1-\varepsilon}$ and consider them separately. More precisely, 
observe that $\Phi(N, n) \supset \bigcup_{0 \leq \ell \leq n^{\varepsilon}-1} \Phi_{\ell}$, where

$$
\Phi_{\ell}=\bigcup_{\substack{k \leq N \\ X_{\ell n^{1-\varepsilon}}^{k} \in B\left(X_{0}^{k}, n^{1 / 2}\right)}}\left\{X_{\ell n^{1-\varepsilon}}^{k}, \ldots, X_{(\ell+1) n^{1-\varepsilon}}^{k}\right\} \cap B\left(X_{0}^{k}, 2 n^{(1-\varepsilon) / 2}\right) .
$$

Using the standard random walk scaling, $P\left[X_{\ell n^{1-\varepsilon}}^{k} \in B\left(X_{0}^{k}, n^{1 / 2}\right)\right]>c$ for all $\ell \in\left\{0, \ldots, n^{\varepsilon}\right\}$, and thus the union contains more than $N^{1-\varepsilon}$ terms with probability at least $1-\mathfrak{s . e} .(n)$. Therefore, using the both estimates of Lemma 6.5 the Markov property, and the assumption of the present lemma, $P\left[\operatorname{cap} \Phi_{\ell} \geq(N n)^{1-\varepsilon} \mid \mathcal{F}_{\ell n^{1-\varepsilon}}\right] \geq c$. $P$-a.s., where $\mathcal{F}_{i}$ is the $\sigma$-algebra generated by $X_{j}^{k}, k \leq N, j \leq i$. As cap $\Phi(N, n) \geq \max _{\ell \leq n^{\varepsilon}} \operatorname{cap} \Phi_{\ell}$, the lemma follows easily from these facts.

Remark 6.7. Lemma 6.6 holds also for $N=1$. An easy adaptation of the previous proof is left as exercise.

We may now prove 6.3 of Proposition 6.2 Let $\left\{w_{1}, \ldots, w_{N}\right\}$ be an arbitrary enumeration of $\operatorname{supp} \mu_{B(n), u}$, that is $w_{k}$ 's are random walk trajectories hitting $B(n)$ with labels smaller than $u$. We will show that for every $k, l$

$$
\mathbb{P}\left[\text { Range } w_{k} \text { and Range } w_{l} \text { are connected within } \mathcal{I}^{u}\right] \geq 1-\mathfrak{s e c}(n) .
$$

By the definition of random interlacements, $N$ has Poisson distribution with parameter $u$ cap $B(n) \sim n^{d-2}$. Therefore, $\mathbb{P}\left[N \geq n^{d}\right]=$ s.e. $(n)$, and thus, by the remark after the definition of $\mathfrak{s e .}(\cdot),(6.26)$ implies 6.3$)$. Without loss of generality, we take $k=1, l=2$ in 6.26$)$.

Let $s=s(d)=\left\lfloor\frac{d}{2}\right\rfloor-1$. We split $\left\{w_{3}, \ldots, w_{N}\right\}$ into $s$ independent Poisson processes,

$$
\mu_{\ell}=\mu_{B(n), \ell u / r}-\mu_{\ell-1}, \quad \ell=1, \ldots, r,
$$

where $\mu_{0}=\delta_{w_{1}}+\delta_{w_{2}}$. As before, $P\left[\left|\operatorname{supp} \mu_{\ell}\right| \geq n^{d-2-\varepsilon}\right]=1-\mathfrak{s . e .}(n)$. For $w \in W$, let $T_{n}(w)=\inf \{k \geq 0: w(k) \notin B(3 n)\}$ and $R(w)=$ $\left\{w(0), \ldots, w\left(n^{2} \wedge T_{n}(w)\right)\right\}$. Set $V_{0}=\left\{w_{1}\right\}, A_{0}=R\left(w_{1}\right)$, and for $\ell=$ $1, \ldots, r$,

$$
\begin{aligned}
& V_{\ell}=\left\{w \in \operatorname{supp} \mu_{\ell}: R(w) \cap A_{\ell-1} \neq \emptyset\right\}, \\
& A_{\ell}=\bigcup_{w \in V_{\ell}} R(w) .
\end{aligned}
$$

We claim that for all $\ell=1, \ldots, r$ and some $\varepsilon>0$ small.

$$
\mathbb{P}\left[\left|V_{\ell}\right| \geq n^{2 \ell-\varepsilon}\right]=1-\mathfrak{s} \cdot \text {. }(n) .
$$


Indeed, this is trivially true for $\left|V_{0}\right|$. If 6.30 holds for $\ell-1$, then by Lemma 6.6

$$
\mathbb{P}\left[\operatorname{cap} A_{\ell-1} \geq n^{(1-\varepsilon)(2(\ell-1)-\varepsilon)} n^{2(1-\varepsilon)}\right]=1-\text { s.e. }(n),
$$

When cap $A_{\ell-1} \geq n^{2 \ell-\varepsilon^{\prime}}$, then by Lemma 6.3 , the probability than one random walk started in $B(n)$ hits $A_{l-1}$ before time $n^{2}$ is bounded from below by $c n^{2 \ell-\varepsilon^{\prime}} n^{2-d}=c n^{2 \ell+2-d-\varepsilon^{\prime}}$. As $\mu_{\ell}$ contains with probability $1-$ s.e. $(n)$, at least $n^{d-2-\varepsilon}$ independent walks, at least $n^{d-2-\varepsilon} n^{2 \ell+2-d-\varepsilon^{\prime}} n^{-\varepsilon}=$ $n^{2 \ell-\bar{\varepsilon}}$ of them hit $A_{\ell-1}$ before the time $n^{2}$, and exit $B(3 n)$, again with $1-\mathfrak{s . e} .(n)$ probability. This completes the proof of 6.30 .

From 6.30 it follows that $\left|V_{s}\right| \geq n^{2 s-\varepsilon} \geq n^{d-3-\varepsilon}$, and, by Lemma 6.6 and Remark 6.7 cap $R\left(w_{2}\right) \geq c n^{2-\varepsilon}$, with $1-$ s.e. $(n)$ probability. Consider now random walks in $V_{s}$. After hitting $A_{s-1}$ the rest of their trajectories is independent of the past, so Lemma 6.3 once more implies that

$$
\left.P\left[\left\{X_{H_{A_{s-1}}}^{k}, \ldots, X_{H_{A_{s-1}}+n^{2}}^{k}\right\} \cap R\left(w_{2}\right)\right] \geq n^{4-d-\varepsilon}\right] .
$$

Hence, the event 'at least one walk in $V_{s}$ hits $R\left(w_{2}\right)$ ' has probability at least $1-$ s.e. $(n)$. This implies 6.26 and completes the proof of 6.3 .

The proof of 6.4 is analogous, it is sufficient to take $w_{2} \in \operatorname{supp} \mu_{B(n), u^{\prime}}$ $\operatorname{supp} \mu_{B(n), u}$.

The proof of Proposition 6.2 that we just finished, has some interesting consequences. We actually proved that the set

$$
\overline{\mathcal{I}}_{n}^{u}:=\bigcup_{w \in \operatorname{supp} \mu_{B(n), u}} R(w)
$$

is with high probability connected. Since $R(w) \subset B(3 n)$, we see $\overline{\mathcal{I}}_{n}^{u} \subset \mathcal{I}_{n}^{u}$ is connected 'locally', that is one does not to go far from $B(n)$ to make a connection. Of course, in general, Range $w \cap B(n) \neq R(w) \cap B(n)$, so we did not show that $\mathcal{I}_{n}^{u} \cap B(n)$ is 'locally connected'. However, it is not difficult to extend the previous techniques to show

$\mathbb{P}\left[\right.$ every $x, y \in B(n) \cap \mathcal{I}^{u}$ are connected in $\left.\mathcal{I}^{u} \cap B(3 n)\right]=1-\mathfrak{s . e} .(n)$.

Another consequence of the previous proof is the following claim. With probability $1-$ s.e. $(n)$, for every pair $w, w^{\prime} \in \operatorname{supp} \mu_{B(n), u}$ there are $w=$ $w_{0}, w_{1}, \ldots, w_{s+1}=w^{\prime} \in \operatorname{supp} \mu_{B(n), u}$ such that $w_{i}$ intersects $w_{i-1}, i=$ $1, \ldots, s+1$. Borel-Cantelli lemma then implies:

Corollary $6.8(d \geq 5)$. Consider (random) graph $\mathcal{G}$ whose vertices are all trajectories in $\operatorname{supp} \mu_{\mathbb{Z}^{d}, u}$, and whose two vertices are connected by an edge iff the corresponding trajectories intersect. Then $\mathbb{P}$-a.s., the diameter of $\mathcal{G}$ satisfies $\operatorname{diam} \mathcal{G} \leq\lfloor d / 2\rfloor$. 
Surprisingly, this bound is optimal when $d$ is odd. The correct upper bound (and in fact also the lower bound) is given in the following theorem.

Theorem $6.9([35,38])$. For every $u>0$, the diameter of $\mathcal{G}$ equals $\lceil d / 2\rceil-$ $1, \mathbb{P}$-a.s.

For even $d$ 's, our upper bound exceeds the correct one by one. The main reason for this is that we decided to prove s.e. $(n)$ bounds on probabilities, which in turn forced us to loose $n^{\varepsilon}$ it many steps. These $n^{\varepsilon}$ 's are what is missing in the last paragraph of the proof of Proposition 6.2 to get the optimal bound.

Almost the same techniques, however, allow to show that (cf. 6.30, (6.32), for all $n$ large enough,

$$
\mathbb{P}\left[\mid V_{\ell} \geq c n^{2 \ell-2}\right]>c^{\prime}>0 \quad \text { and } \quad \mathbb{P}\left[w_{2} \text { intersects } A_{s}\right]>c^{\prime} .
$$

In 38 , estimates similar to 6.35 together with ideas inspired by Wiener test (see [27, Theorem 2.2.5]) are applied to obtain the optimal upper bound.

Our proof of Proposition 6.2 has yet another consequence on what is called chemical distance on the interlacement set, defined for $x, y \in \mathcal{I}^{u}$ by

$$
\begin{aligned}
& \rho_{u}(x, y)=\min \left\{n: \exists x=z_{0}, z_{1}, \ldots, z_{n}=y\right. \text { such that } \\
& \left.\qquad z_{i} \in \mathcal{I}^{u} \text { and }\left|z_{i}-z_{i-1}\right|=1, i=1, \ldots, n\right\} .
\end{aligned}
$$

Claim 6.34 easily yields,

$$
\mathbb{P}\left[\rho_{u}(x, y) \leq 9 n^{d} \forall x, y \in B(n) \cap \mathcal{I}^{u}\right]=1-\mathfrak{s e} \text {. }(n) .
$$

The bound $9 n^{d}$ is of course far from being optimal. (6.37) is however one of the ingredients of the optimal upper bound $C n$ which is proved in [12]:

Theorem 6.10 ([12]). For every $u>0$ and $d \geq 3$ there exist constants $C, C^{\prime}<\infty$ and $\delta \in(0,1)$ such that

$\mathbb{P}\left[\right.$ there exists $x \in \mathcal{I}^{u} \cap[-n, n]^{d}$ such that $\left.\rho_{u}(0, x)>C n \mid 0 \in \mathcal{I}^{u}\right] \leq C^{\prime} e^{-n^{\delta}}$.

\subsection{Notes}

The properties of interlacement set $\mathcal{I}^{u}$ on $\mathbb{Z}^{d}$ were investigated already in the paper [47. A weaker version of Theorem 6.1] is proved there (see (2.21) in [47]), namely, for every $u>0$,

$$
\mathbb{P}\left[\mathcal{I}^{u} \text { is connected }\right]=1,
$$

which is sufficient to deduce the absence of the phase transition. The proof of [47] is based on the Burton-Keane argument [11]. 
Theorem 6.1 states that for every $u>0, \mathcal{I}^{u}$ is supercritical. It is thus natural to ask, as in the Bernoulli-percolation theory, to which extend the geometry of $\mathcal{I}^{u}$ (at large scales) resembles the geometry of the complete lattice $\mathbb{Z}^{d}$.

Many results going in this direction appeared recently: In [36] it was proved that $\mathcal{I}^{u}$ percolates in two-dimensional slabs, namely that for every $u>0$ and $d \geq 3$ exists $R>0$ such that $\mathcal{I}^{u} \cap\left(\mathbb{Z}^{2} \times[0, R]^{d-2}\right)$ contains an infinite component $\mathbb{P}$-a.s. In [37] it was further shown that the random walk on $\mathcal{I}^{u}$ is $\mathbb{P}$-a.s. transient. Theorem 6.10 is another result of this type.

Theorem 6.9 giving the diameter of the graph $\mathcal{G}$, was independently shown by Procaccia and Tykesson [35] using ideas of stochastic dimension theory developed in [5], and by R $\tilde{A}_{i}$ th and Sapozhnikov [38] whose methods are commented above.

The techniques used in our proof of Proposition 6.2 are mixture of methods from [38] (where we borrowed Lemmas 6.4 and 6.5 and [12] (which contains contains many s.e. $(n)$ estimates $)$.

The results of this chapter can be used to deduce some properties of the trace $\left\{X_{0}, \ldots, X_{u N^{d}}\right\}$ of the simple random walk on the torus. E.g., Theorem 6.10 was combined in [12] with the coupling of random interlacements and random walk from [53] to control the chemical distance on the random walk trace. 


\section{Chapter 7}

\section{Locally tree-like graphs}

In the previous chapters we have studied the random walk on the torus and the corresponding interlacement set on $\mathbb{Z}^{d}$. We have seen that in that case many interesting questions are still open, including the existence of a sharp phase transition in the connectivity of the vacant set of the random walk, and its correspondence to the phase transition of random interlacements. Answering these questions requires a better control of the random interlacements in both sub-critical and supercritical phase which is not available at present.

In this chapter we are going to explore random interlacements on graphs where such control is available, namely on trees. We will then explain how such control can be used to show the phase transition for the vacant set of random walk on finite 'locally tree-like' graphs, and to give the equivalence of critical points in both models. In other worlds, we prove Conjecture 2.6 on the locally tree-like graphs.

\subsection{Random interlacements on trees}

We start by considering random interlacements on trees. We will show that vacant clusters of this model behave like Galton-Watson trees, which allows us to perform many exact computations. As in this lecture notes we only deal with random walks and random interlacements on regular graphs, we restrict our attention to regular trees only.

Let $\mathbb{T}_{d}$ be infinite $d$-regular tree, $d \geq 3$, for which the simple random walk is transient. We may therefore define random interlacements on $\mathbb{T}_{d}$ similarly as we did for $\mathbb{Z}^{d}$.

We write $P_{x}$ for the law of the canonical simple random walk $\left(X_{n}\right)$ on $\mathbb{T}_{d}$ started at $x \in \mathbb{T}_{d}$, and denote by $e_{K}, K \subset \subset \mathbb{T}_{d}$ the equilibrium measure,

$$
e_{K}(x)=P_{x}\left[\tilde{H}_{K}=\infty\right] \mathbf{1}\{x \in K\} .
$$


Observe that if $K$ is connected, $e_{K}$ can be easily computed. Indeed, denoting by $d(\cdot, \cdot)$ the graph distance on the tree, we observe that under $P_{x}$, the process $d\left(X_{n}, x\right)$ has the same law as a drifted random walk on $\mathbb{N}$ started at 0 . If not at 0 , this walk jumps to the right with probability $(d-1) / d$ and to the left with probability $1 / d$; at 0 it goes always to the right. Using standard computation for the random walk with drift, see e.g. [58, Lemma 1.24, it is then easy to show that

$$
P_{x}\left[\tilde{H}_{x}=\infty\right]=P_{y}\left[H_{x}=\infty\right]=\frac{d-2}{d-1},
$$

for every neighbor $y$ of $x$. For $K$ connected, we then get

$$
e_{K}(x)=\frac{1}{d} \#\{y: y \sim x, y \notin K\} \frac{d-2}{d-1},
$$

where the first two terms give the probability that the first step of the random walk exists $K$.

We consider spaces $W_{+}, W, W^{\star}, \Omega$ and measures $Q_{K}$ defined similarly as in Chapter 3 , replacing $\mathbb{Z}^{d}$ by $\mathbb{T}_{d}$ in these definitions when appropriate. As in Theorem 3.1, it can be proved that there exists a unique $\sigma$-finite measure $\nu$ on $\left(W^{\star}, \mathcal{W}^{\star}\right)$ satisfying the restriction property (3.14). Using this measure, we can then construct a Poisson point process $\omega$ on $W^{\star} \times \mathbb{R}_{+}$ with intensity measure $\nu\left(\mathrm{d} w^{\star}\right) \otimes \mathrm{d} u$ and define the interlacements at level $u$ and its vacant set as in (3.27), (3.28).

The main result of this section is the following theorem.

Theorem 7.1 ([50, Theorem 5.1). Let $x \in \mathbb{T}_{d}$ and define $f_{x}: \mathbb{T}_{d} \rightarrow[0,1]$ by

$$
\begin{aligned}
f_{x}(z)= & P_{z}\left[d\left(X_{n}, x\right)>d(x, z) \text { for all } n>0\right] \\
& \times P_{z}\left[d\left(X_{n}, x\right) \geq d(x, z) \text { for all } n \geq 0\right] .
\end{aligned}
$$

Then the vacant cluster of $\mathcal{V}^{u}$ containing $x$ in the random interlacements has the same law as the open cluster containing $x$ in the inhomogeneous independent Bernoulli site percolation on $\mathbb{T}_{d}$ characterized by

$$
\operatorname{Prob}[z \text { is open }]=\exp \left\{-u f_{x}(z)\right\} \text {. }
$$

Remark 7.2. 1. Observe that on $\mathbb{T}_{d}, f_{z}(x)$ is the same for all $z \neq x$. Hence, the cluster of $\mathcal{V}^{u}$ containing $x$ can be viewed as a Galton-Watson tree with a particular branching law in the first generation.

2. Beware that the joint law of, e.g., vacant clusters containing two points $x \neq y \in \mathbb{T}_{d}$ is not the same as in the Bernoulli percolation.

Proof. We partition the space $W^{\star}$ into disjoint subsets $W^{\star, z}$ according to the position where $w^{\star} \in W^{\star}$ get closest to the given point $x$,

$$
W^{\star}=\bigcup_{z \in \mathbb{T}_{d}} W^{\star, z},
$$


where

$$
W^{\star, z}=\left\{w^{\star} \in W^{\star}: z \in \operatorname{Range}\left(w^{\star}\right), d\left(x, \operatorname{Range}\left(w^{\star}\right)\right)=d(x, z)\right\} .
$$

(The fact that $W^{\star, z}$ are disjoint follows easily from the fact that $\mathbb{T}_{d}$ is a tree.)

As a consequence of disjointness we obtain that the random variables $\omega\left(W^{\star, z} \times[0, u]\right)$ are independent. We may thus define independent site Bernoulli percolation on $\mathbb{T}_{d}$ by setting

$$
Y_{z}^{u}(\omega)=\mathbf{1}\left\{\omega\left(W^{\star, z} \times[0, u]\right) \geq 1\right\} \quad \text { for } z \in \mathbb{T}_{d} .
$$

By (3.9), 3.14 and (7.7), we see that

$$
\mathbb{P}\left[Y_{z}^{u}=0\right]=\exp \left\{-u f_{x}(z)\right\} .
$$

To finish the proof of the theorem, it remains to observe that the null cluster of $\left(Y^{u}\right)$ containing $x$ coincides with the component of $\mathcal{V}^{u}$ containing $x$. The easy proof of this claim is left as exercise.

As a corollary of Theorem 7.1 and 7.3 we obtain the value of critical threshold of random interlacements on $\mathbb{T}_{d}$ which, similarly as on $\mathbb{Z}^{d}$, is defined by

$$
u_{\star}\left(\mathbb{T}_{d}\right)=\inf \left\{u \geq 0: \mathbb{P}\left[\text { the cluster of } x \text { in } \mathcal{V}^{u} \text { is infinite }\right]=0\right\} .
$$

Corollary 7.3. The critical point of the random interlacements on $\mathbb{T}_{d}$ is given by

$$
u_{\star}\left(\mathbb{T}_{d}\right)=\frac{d(d-1) \log (d-1)}{(d-2)^{2}} .
$$

Proof. For $z \neq x$, by considering drifted random walk as above (7.1), it is easy to see that

$$
f_{x}(z)=\frac{d-2}{d-1} \times \frac{d-1}{d} \frac{d-2}{d-1}=\frac{(d-2)^{2}}{d(d-1)} .
$$

Hence, the offspring distribution of the Galton-Watson process mentioned in Remark 7.2 is (except in the first generation) binomial with parameters $\left(d-1, \exp \left\{-u \frac{(d-2)^{2}}{d(d-1)}\right\}\right)$. This Galton-Watson process is critical if the mean of its offspring distribution is equal one, implying that $u_{\star}\left(\mathbb{T}_{d}\right)$ is the solution of

$$
(d-1) \exp \left\{-u \frac{(d-2)^{2}}{d(d-1)}\right\}=1,
$$

yielding 7.11. 
Remark 7.4. For the previous result, the offspring distribution in the first generation is irrelevant. Using (7.1) and Theorem 7.1 it is however easy to see that (for $k=0, \ldots, d$ )

$$
\begin{gathered}
\mathbb{P}\left[x \in \mathcal{V}^{u}\right]=e^{-u \operatorname{cap}(x)}=e^{-u f_{x}(x)}=e^{-u(d-2) /(d-1)}, \\
\mathbb{P}\left[\left|\mathcal{V}^{u} \cap\{y: y \sim x\}\right|=k \mid x \in \mathcal{V}^{u}\right]=\left(\begin{array}{l}
d \\
k
\end{array}\right) e^{-u k \frac{(d-2)^{2}}{d(d-1)}}\left(1-e^{-u\left(\frac{d-2}{d(d-1)}\right)}\right)^{d-k}
\end{gathered}
$$

We will need these formulas later.

\subsection{Random walk on tree-like graphs}

We now return to the problem of the vacant set of the random walk on finite graphs. However, instead of considering the torus as in Chapter 2 we are going to study graphs that locally look like a tree, so that we can use the results of the previous section.

Actually, most of this section will deal with so-called random regular graphs. A random $d$-regular graph with $n$ vertices is a graph that is chosen uniformly from the set $\mathcal{G}_{n, d}$ of all simple (i.e. without loops and multiple edges) graphs with the vertex set $V_{n}=[n]:=\{1, \ldots, n\}$ and all vertices of degree $d$, assuming tacitly that $n d$ is even. We let $\mathbb{P}_{n, d}$ to denote the distribution of such graph, that is the uniform distribution on $\mathcal{G}_{n, d}$.

It is well-known that with probability tending to 1 as $n$ increases, the majority of vertices in a random regular graph has a neighborhood with radius $c \log n$ which is graph-isomorph to a ball in $\mathbb{T}_{d}$, see Lemma 3.2 of [30.

For a fixed graph $G=(V, \mathcal{E})$ let $P^{G}$ be the law of random walk on $G$ started from the uniform distribution and $\left(X_{t}\right)_{t \geq 0}$ the canonical process. As before we will be interested in the vacant set

$$
\mathcal{V}_{G}^{u}=V \backslash\left\{X_{t}: 0 \leq t \leq u|V|\right\},
$$

and denote by $\mathcal{C}_{\max }^{u}$ its maximal connected component.

We will study the properties of the vacant set under the annealed measure $\mathbf{P}_{n, d}$ given by

$$
\mathbf{P}_{n, d}(\cdot)=\int P^{G}(\cdot) \mathbb{P}_{n, d}(\mathrm{~d} G)\left(=\sum_{G} P^{G}(\cdot) \mathbb{P}_{n, d}[G]\right) .
$$

The following theorem states that a phase transition occurs in the behavior of the vacant set on random regular graph.

Theorem $7.5\left(d \geq 3, u_{\star}:=u_{\star}\left(\mathbb{T}_{d}\right)\right)$. 
(a) For every $u<u_{\star}$ there exist constant $c(u) \in(0,1)$ such that

$$
n^{-1}\left|\mathcal{C}_{\max }\right| \stackrel{n \rightarrow \infty}{\longrightarrow} c(u) \quad \text { in } \mathbf{P}_{n, d} \text {-probability }
$$

(b) When $u>u_{\star}$, then for every $\varepsilon$ there is $K(u, \varepsilon)<\infty$ such that for all $n$ large

$$
\mathbf{P}_{n, d}\left[\left|\mathcal{C}_{\max }\right| \geq K(u, \varepsilon) \log n\right] \leq \varepsilon .
$$

Observe that Theorem 7.5 not only proves the phase transition, but also confirms that the critical point coincides with the critical point of random interlacements on $\mathbb{T}_{d}$. The theorem was proved (in a weaker form but for a larger class of graphs) in [14]. In these notes, we are going to use a simple proof given by Cooper and Frieze [15] which uses in a clever way the randomness of the graph. Besides being simple, this proof has an additional advantage that it can be used also in the vicinity of the critical point: By a technique very similar to the ones presented here, it was proved in 13 , that the vacant set of the random walk exhibits a doublejump behavior analogous to the maximal connected cluster in Bernoulli percolation:

\section{Theorem 7.6.}

(a) Critical window. Let $\left(u_{n}\right)_{n \geq 1}$ be a sequence satisfying

$$
\left|n^{1 / 3}\left(u_{n}-u_{\star}\right)\right| \leq \lambda<\infty \text { for all } n \text { large enough }
$$

Then for every $\varepsilon>0$ there exists $A=A(\varepsilon, d, \lambda)$ such that for all $n$ large enough

$$
\mathbf{P}_{n, d}\left[A^{-1} n^{2 / 3} \leq\left|\mathcal{C}_{\max }^{u_{n}}\right| \leq A n^{2 / 3}\right] \geq 1-\varepsilon
$$

(b) Above the window. When $\left(u_{n}\right)_{n \geq 1}$ satisfies

$$
u_{\star}-u_{n} \stackrel{n \rightarrow \infty}{\longrightarrow} 0, \quad \text { and } \quad n^{1 / 3}\left(u_{\star}-u_{n}\right) \stackrel{n \rightarrow \infty}{\longrightarrow} \infty,
$$

then

$$
\left|\mathcal{C}_{\max }^{u_{n}}\right| / n^{2 / 3} \stackrel{n \rightarrow \infty}{\longrightarrow} \infty, \quad \text { in } \mathbf{P}_{n, d} \text {-probability }
$$

(c) Below the window. When $\left(u_{n}\right)_{n \geq 1}$ satisfies

$$
u_{\star}-u_{n} \stackrel{n \rightarrow \infty}{\longrightarrow} 0, \quad \text { and } \quad n^{1 / 3}\left(u_{\star}-u_{n}\right) \stackrel{n \rightarrow \infty}{\longrightarrow}-\infty,
$$

then

$$
\left|\mathcal{C}_{\text {max }}^{u_{n}}\right| / n^{2 / 3} \stackrel{n \rightarrow \infty}{\longrightarrow} 0 . \quad \text { in } \mathbf{P}_{n, d} \text {-probability } .
$$

We will now sketch the main steps of the proof of Theorem 7.5 . Detailed proofs can be found in [15, 13]. 


\subsubsection{Very short introduction to random graphs}

We start by reviewing some properties of random regular graphs, for more about these graphs see e.g. [7, 59.

It turns out that it is easier to work with multi-graphs instead of simple graphs. Therefore we introduce $\mathcal{M}_{n, d}$ for the set of all $d$-regular multigraphs with vertex set $[n]$.

For reasons that will be explained later, we also define random graphs with a given degree sequence $\boldsymbol{d}:[n] \rightarrow \mathbb{N}$. We will use $\mathcal{G}_{\boldsymbol{d}}$ to denote the set of graphs for which every vertex $x \in[n]$ has the degree $\boldsymbol{d}_{x}=$ $\boldsymbol{d}(x)$. Similarly, $\mathcal{M}_{\boldsymbol{d}}$ stands for the set of such multi-graphs; here loops are counted twice when considering the degree. $\mathbb{P}_{n, d}$ and $\mathbb{P}_{\boldsymbol{d}}$ denote the uniform distributions on $\mathcal{G}_{n, d}$ and $\mathcal{G}_{\boldsymbol{d}}$ respectively. We say that a given event holds asymptotically almost surely (denoted by $\mathbb{P}_{n, d}$-a.a.s. or $\mathbb{P}_{\boldsymbol{d}^{-}}$ a.a.s depending on the case) if it holds with probability converging to one, with respect to $\mathbb{P}_{n, d}$ or $\mathbb{P}_{\boldsymbol{d}}$.

We first introduce the pairing construction, which allows to generate graphs distributed according to $\mathbb{P}_{n, d}$, starting from a random pairing of a set with $d n$ elements. The same construction can be used to generate a random graph chosen uniformly at random from $\mathcal{G}_{\boldsymbol{d}}$.

We consider a sequence $\boldsymbol{d}: V_{n} \rightarrow \mathbb{N}$ such that $\sum_{x \in V_{n}} \boldsymbol{d}_{x}$ is even. Given such a sequence, we associate to every vertex $x \in V_{n}, \boldsymbol{d}_{x}$ half-edges. The set of half-edges is denoted by $H_{\boldsymbol{d}}=\left\{(x, i): x \in V_{n}, i \in\left[\boldsymbol{d}_{x}\right]\right\}$. We write $H_{n, d}$ for the case $\boldsymbol{d}_{x}=d$ for all $x \in V_{n}$. Every perfect matching $M$ of $H_{\boldsymbol{d}}$ (i.e. partitioning of $H_{\boldsymbol{d}}$ into $\left|H_{\boldsymbol{d}}\right| / 2$ disjoint pairs) corresponds to a multi-graph $G_{M}=\left(V_{n}, \mathcal{E}_{M}\right) \in \mathcal{M}_{\boldsymbol{d}}$ with

$$
\mathcal{E}_{M}=\left\{\{x, y\}:\{(x, i),(y, j)\} \in M \text { for some } i \in\left[\boldsymbol{d}_{x}\right], j \in\left[\boldsymbol{d}_{y}\right]\right\} .
$$

We say that the matching $M$ is simple, if the corresponding multi-graph $G_{M}$ is simple, that is $G_{M}$ is a graph. With a slight abuse of notation, we write $\overline{\mathbb{P}}_{\boldsymbol{d}}$ for the uniform distribution on the set of all perfect matchings of $H_{\boldsymbol{d}}$, and also for the induced distribution on the set of multi-graphs $\mathcal{M}_{\boldsymbol{d}}$. It is well known (see e.g. [7] or [31]) that a $\overline{\mathbb{P}}_{\boldsymbol{d}}$ distributed multi-graph $G$ conditioned on being simple has distribution $\mathbb{P}_{\boldsymbol{d}}$, that is

$$
\overline{\mathbb{P}}_{\boldsymbol{d}}\left[G \in \cdot \mid G \in \mathcal{G}_{\boldsymbol{d}}\right]=\mathbb{P}_{\boldsymbol{d}}[G \in \cdot],
$$

and that, for $d$ constant, there is $c>0$ such that for all $n$ large enough

$$
c<\overline{\mathbb{P}}_{n, d}\left[G \in \mathcal{G}_{n, d}\right]<1-c .
$$

These two claims allow to deduce $\mathbb{P}_{n, d^{-}}$a.a.s. statements directly from $\overline{\mathbb{P}}_{n, d^{-}}$ a.a.s. statements.

The main advantage of dealing with matchings is that they can be constructed sequentially: To construct a uniformly distributed perfect matching of $H_{\boldsymbol{d}}$ one samples without replacements a sequence $h_{1}, \ldots, h_{\left|H_{\boldsymbol{d}}\right|}$ of 
elements of $H_{\boldsymbol{d}}$ in the following way. For $i$ odd, $h_{i}$ can be chosen by an arbitrary rule (which might also depend on the previous $\left(h_{j}\right)_{j<i}$ ), while if $i$ is even, $h_{i}$ must be chosen uniformly among the remaining half-edges. Then, for every $1 \leq i \leq\left|H_{\boldsymbol{d}}\right| / 2$ one matches $h_{2 i}$ with $h_{2 i-1}$.

It is clear from the above construction that, conditionally on $M^{\prime} \subseteq M$ for a (partial) matching $M^{\prime}$ of $H_{\boldsymbol{d}}, M \backslash M^{\prime}$ is distributed as a uniform perfect matching of $H_{\boldsymbol{d}} \backslash\left\{(x, i):(x, i)\right.$ is matched in $\left.M^{\prime}\right\}$. Since the law of the graph $G_{M}$ does not depend on the labels ' $i$ ' of the half-edges, we obtain for all partial matchings $M^{\prime}$ of $H_{\boldsymbol{d}}$ the following restriction property,

$$
\overline{\mathbb{P}}_{\boldsymbol{d}}\left[G_{M \backslash M^{\prime}} \in \cdot \mid M \supset M^{\prime}\right]=\overline{\mathbb{P}}_{\boldsymbol{d}^{\prime}}\left[G_{M} \in \cdot\right],
$$

where $\boldsymbol{d}_{x}^{\prime}$ is the number of half-edges incident to $x$ in $H_{\boldsymbol{d}}$ that are not yet matched in $M^{\prime}$, that is

$$
\boldsymbol{d}_{x}^{\prime}=\boldsymbol{d}_{x}-\left|\left\{\left\{\left(y_{1}, i\right),\left(y_{2}, j\right)\right\} \in M^{\prime}: y_{1}=x, i \in\left[\boldsymbol{d}_{x}\right]\right\}\right|,
$$

and $G_{M \backslash M^{\prime}}$ is the graph corresponding to a non-perfect matching $M \backslash M^{\prime}$, defined in the obvious way.

\subsubsection{Distribution of the vacant set}

We now study the properties of the vacant set of random walk. Instead of the vacant set, it will be more convenient to consider the following object that we call vacant graph $\mathbf{V}^{u}$. It is defined by $\mathbf{V}^{u}=\left(V, \mathcal{E}^{u}\right)$ with

$$
\mathcal{E}^{u}=\left\{\{x, y\} \in \mathcal{E}: x, y \in \mathcal{V}_{G}^{u}\right\} .
$$

It is important to notice that the vertex set of $\mathbf{V}^{u}$ is a deterministic set $V$ and not the random set $\mathcal{V}_{G}^{u}$. In particular $\mathbf{V}^{u}$ is not the graph induced by $\mathcal{V}_{G}^{u}$ in $G$. Observe however that the maximal connected component of the vacant set $\mathcal{C}_{\max }$ (defined before in terms of the graph induced by $\mathcal{V}^{u}$ in $G$ ) coincides with the maximal connected component of the vacant graph $\mathbf{V}^{u}$ (except when $\mathcal{V}^{u}$ is empty, but this difference can be ignored in our investigations).

We use $\mathcal{D}^{u}: V \rightarrow \mathbb{N}$ to denote the (random) degree sequence of $\mathbf{V}^{u}$, and write $Q_{n, d}^{u}$ for the distribution of this sequence under the annealed measure $\overline{\mathbf{P}}_{n, d}$, defined by $\overline{\mathbf{P}}_{n, d}(\cdot):=\int P^{G}(\cdot) \overline{\mathbb{P}}_{n, d}(\mathrm{~d} G)\left(=\sum_{G} P^{G}(\cdot) \overline{\mathbb{P}}_{n, d}[G]\right)$.

The following important but simple observation from [15] allows to reduce questions on the properties of the vacant set $\mathcal{V}^{u}$ of the random walk on random regular graphs to questions on random graphs with given degree sequences.

Proposition 7.7 (Lemma 6 of [15]). For every $u \geq 0$, the distribution of the vacant graph $\mathbf{V}^{u}$ under $\overline{\mathbf{P}}_{n, d}$ is given by $\overline{\mathbb{P}}_{\boldsymbol{d}}$ where $\boldsymbol{d}$ is sampled 
according to $Q_{n, d}^{u}$, that is

$$
\overline{\mathbf{P}}_{n, d}\left[\mathbf{V}^{u} \in \cdot\right]=\int \overline{\mathbb{P}}_{\boldsymbol{d}}[G \in \cdot] Q_{n, d}^{u}(\mathrm{~d} \boldsymbol{d})\left(=\sum_{\boldsymbol{d}} \mathbb{P}_{\boldsymbol{d}}[G \in \cdot] Q_{n, d}^{u}(\boldsymbol{d})\right)
$$

Proof. The full proof is given in [15] and [13], here we give a less rigorous but more transparent explanation. The main observation behind this proof is the following joint construction of a $\overline{\mathbf{P}}_{n, d}$ distributed multi-graph and a (discrete-time) random walk on it.

1. Pick $X_{0}$ in $V$ uniformly.

2. Pair all half-edges incident to $X_{0}$ according to the pairing construction given above.

3. Pick uniformly a number $Z_{0}$ in $[d]$ and set $X_{1}$ to be the vertex paired with $\left(X_{0}, Z_{0}\right)$.

4. Pair all not-yet paired half-edges incident to $X_{1}$ according to the pairing construction.

5. Pick uniformly a number $Z_{1}$ in $[d]$ and set $X_{2}$ to be the vertex paired with $\left(X_{1}, Z_{1}\right)$.

6. Repeat the above steps for $X_{i+1}$ and

7. Stop when $X_{|V| u}$ and its neighbors are known.

At this moment we have constructed the first $|V| u$ steps of the random walk trajectory and determined all edges in the graph that are incident to vertices visited by this trajectory. To finish the construction of the graph we should

8. Pair all remaining half-edges according to the pairing construction.

It is not hard to observe that the edges created in step (8) are exactly the edges of the vacant graph $\mathbf{V}^{u}$ and that the degree of $x$ in $\mathbf{V}^{u}$ is known already at step (7). Using the restriction property of partial matchings 7.29 , it is then not difficult to prove the proposition.

Due to the last proposition, in order to show Theorem 7.5 we need an information about two objects: the maximal connected component of a

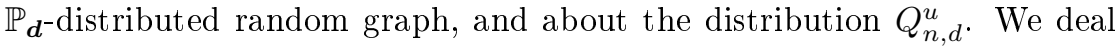
with them in the next two subsections. 


\subsubsection{Random graphs with a given degree sequence.}

The random graphs with a given (deterministic) degree sequence are well studied. A rather surprising fact, due to Molloy and Reed [33] is that the phase transition in its behavior is characterized by a single real parameter computed from a degree sequence. We give a very weak version of result of 33 :

Theorem 7.8. For a degree sequence $\boldsymbol{d}:[n] \rightarrow \mathbb{N}$, let

$$
Q(d)=\frac{\sum_{x=1}^{n} \boldsymbol{d}_{x}^{2}}{\sum_{x=1}^{n} \boldsymbol{d}_{x}}-2 .
$$

Consider now a sequence of degree sequences $\left(\boldsymbol{d}^{n}\right)_{n \geq 1}, \boldsymbol{d}^{n}:[n] \rightarrow \mathbb{N}$, and assume that the degrees $d_{x}^{n}$ are uniformly bounded by some $\Delta$ and that $\left|\left\{x \in[n]: d_{x}^{n}=1\right\}\right| \geq \zeta$ for $a \zeta>0$. Then

- If $\lim \inf Q\left(\boldsymbol{d}^{n}\right)>0$, then there is $c>0$ such that with $\overline{\mathbf{P}}_{\boldsymbol{d}}$ probability tending to one the maximal connected component of the graph is larger than $\mathrm{cn}$.

- When $\lim \sup Q\left(\boldsymbol{d}^{n}\right)<0$, then the size of the maximal connected

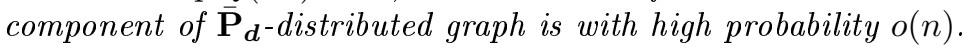

Later works, see e.g. [24, 22, give a more detailed description of random graphs with given degree sequences, including the description of the critical window which allows to deduce Theorem 7.6 .

\subsubsection{The degree sequence of the vacant graph}

We will show that the distribution of the degree sequence of the vacant graph is the same as the distribution of the number of vacant neighbors of any given vertex $x$ in a random interlacements on $\mathbb{T}_{d}$. More precisely, it follows from Remark 7.4 that the probability that $x \in \mathcal{V}_{\mathbb{T}_{d}}^{u}$ and its degree in $\mathcal{V}_{\mathbb{T}^{d}}^{u}$ is $i, i=0, \ldots, d$, is given by

$$
d_{i}^{u}:=e^{-u \frac{d-2}{d-1}}\left(\begin{array}{l}
d \\
i
\end{array}\right) p_{u}^{i}\left(1-p_{u}\right)^{d-i},
$$

with $p_{u}=\exp \left\{-u \frac{(d-2)^{2}}{d(d-1)}\right\}$.

Recall $\mathcal{D}^{u}$ denotes the degree sequence of the vacant graph $\mathbf{V}^{u}$. For any degree sequence $\boldsymbol{d}, n_{i}(\boldsymbol{d})$ denotes the number of vertices with degree $i$ in $\boldsymbol{d}$. The following theorem states that quenched expectation of $n_{i}\left(\mathcal{D}^{u}\right)$ concentrates around $n d_{i}^{u}$.

Theorem 7.9. For every $u>0$ and every $i \in\{0, \ldots, d\}$,

$$
\left|E^{G}\left[n_{i}\left(\mathcal{D}^{u}\right)\right]-n d_{i}^{u}\right| \leq c\left(\log ^{5} n\right) n^{1 / 2}, \quad \overline{\mathbb{P}}_{n, d^{-}} \text {a.a.s. }
$$


We decided not to present the proof of this theorem in these notes, as it uses very similar arguments as the proofs in Chapter 2, The full proof can be found in 553 .

In order to control $Q_{n, d}^{u}$ we need to show that $n_{i}\left(\mathcal{D}^{u}\right)$ concentrates around its mean. This is the result of the following theorem that holds for deterministic graphs.

Theorem 7.10. Let $G$ be a d-regular (multi)graph on $n$ vertices whose spectral gap ${ }^{1} \lambda_{G}$ is larger than some $\alpha>0$. Then, for every $\varepsilon \in\left(0, \frac{1}{4}\right)$, and for every $i \in\{0, \ldots, d\}$,

$$
P^{G}\left[\left|n_{i}\left(\mathcal{D}^{u}\right)-E^{G}\left[n_{i}\left(\mathcal{D}^{u}\right)\right]\right| \geq n^{1 / 2+\varepsilon}\right] \leq c_{\alpha, \varepsilon} e^{-c_{\alpha, \varepsilon} n^{\varepsilon}} .
$$

The proof of this theorem uses concentration inequalities for Lipschitz functions of sequences of not-independent random variables, and can be found in $[13$.

From Theorems 7.9 and 7.10 it is easy to compute the typical value of $Q\left(\mathcal{D}^{u}\right)$. It turns out that it is positive when $u<u_{\star}$ and negative when $u>u_{\star}$. This proves via Theorem 7.8 and Proposition 7.7 the existence of a phase transition of the vacant set.

In fact, the above results allow to compute $Q\left(\mathcal{D}^{u}\right)$ up to an additive error which is $o\left(n^{-1 / 2+\varepsilon}\right)$. This precision is more than enough to apply the stronger results on the behavior of random graphs with given degree sequences [22] and to show Theorem 7.6 .

\subsection{Notes}

Theorem 7.1. that is the comparison of the cluster of the vacant set containing a given point with the cluster of Bernoulli percolation, can be generalized to arbitrary locally finite (weighted) trees. However, as the invariant measure of the random walk is then in general not uniform, a slight care should be taken in defining the random interlacements, see [50].

Apart $\mathbb{T}_{d}$, there is to our knowledge only one other case where the critical value of random interlacements can be computed explicitly (and is nontrivial), namely for the base graph being a Galton-Watson tree. In this case, it was shown in [49] that $u_{\star}$ is a.s. constant (i.e. 'does not depend' on the realization of the Galton-Watson tree) and can be computed implicitly as a solution to a particular equation.

As we have already mentioned, a result very similar to Theorem 7.5 was first proved in [14] (see Theorems 1.2 and 1.3). In [14, the proof strategy follows a description of the local measure, with a flavor similar to that of Chapter 2, therefore it is very different from the techniques sketched

\footnotetext{
${ }^{1}$ Spectral gap $\lambda_{G}$ of a graph $G$ is defined as the spectral gap of the transition matrix of the lazy random on $G$, see 2.2 .
} 
in this chapter. Although we believe that the local picture approach can hardly work to the level of precision obtained in Theorem 7.6, the class of graphs for which this technique applies is much broader: Let us informally mention that Theorems 1.2 and 1.3 of [14] apply to any sequence of graphs $\left(G_{n}\right)_{n \geq 1}$ such that for each $n \geq 1$,

- $G_{n}$ are $d$-regular,

- the spectral gap of $G_{n}$ is bounded from below by a constant and

- there exists an $\epsilon>0$ such that every ball $B(x, \epsilon \log n)$ in $G_{n}$ has at most one cycle.

In 52 the interlacement set $\mathcal{I}^{u}$ was studied in various different graphs. There, special emphasis was given to the connectivity of $\mathcal{I}^{u}$ on different classes of graphs. More precisely, it was proved that

if $G$ is a vertex-transitive, non-amenable graph, then, for $u>0$ small enough,

the interlacement set $I^{u}$ is almost surely disconnected.

On the other hand,

if $G$ is a vertex-transitive, amenable, transient graph, then, for all $u \geq 0, \mathcal{I}^{u}$ is a.s. connected.

An interesting example is given by the product $\mathbb{T}^{d} \times \mathbb{Z}^{d^{\prime}}$ of a $d$-regular tree with a $d^{\prime}$-dimensional lattice, with $d \geq 3$ and $d^{\prime} \geq 1$. In this case, we know that there is a transition between a disconnected and a connected phase for $\mathcal{I}^{u}$ as $u$ crosses a critical threshold $u_{c}$. It is worth mentioning that this critical value has been proved in 55 to be unique, despite the fact that connectivity is not a monotone property. 


\section{Index}

annealed measure, 63

Bernoulli (site) percolation, 40

canonical coordinates, 22

canonical shift, 28

capacity, 15

critical window, 64

Dirac measure, 23

disconnection of cylinder, 24

disconnection time, 25

domination, 42

entrance time, 14

equilibrium distribution, 11

equilibrium measure, 15

excursion, 11

excursions, 19

finite energy, 41

hitting time, 14

interlacement set, 32

Lebesgue measure, 30

local picture, $10,11,13,15$

pairing construction, 65

random interlacements, 29,32

random regular graph, 63

random walk

lazy, 13

simple, 13

transient, 15 regeneration time, 14

regular graph, 60

regular tree, 60

restriction property, 66

reversible, 13

shift operator, 14

spectral gap, 14

time

departure, 19

entrance, 28

exit, 28

return, 19

trajectories

modulo time-shift, 29

trajectory

doubly-infinite, 28

vacant set, 33

vacant set in the torus, 9 


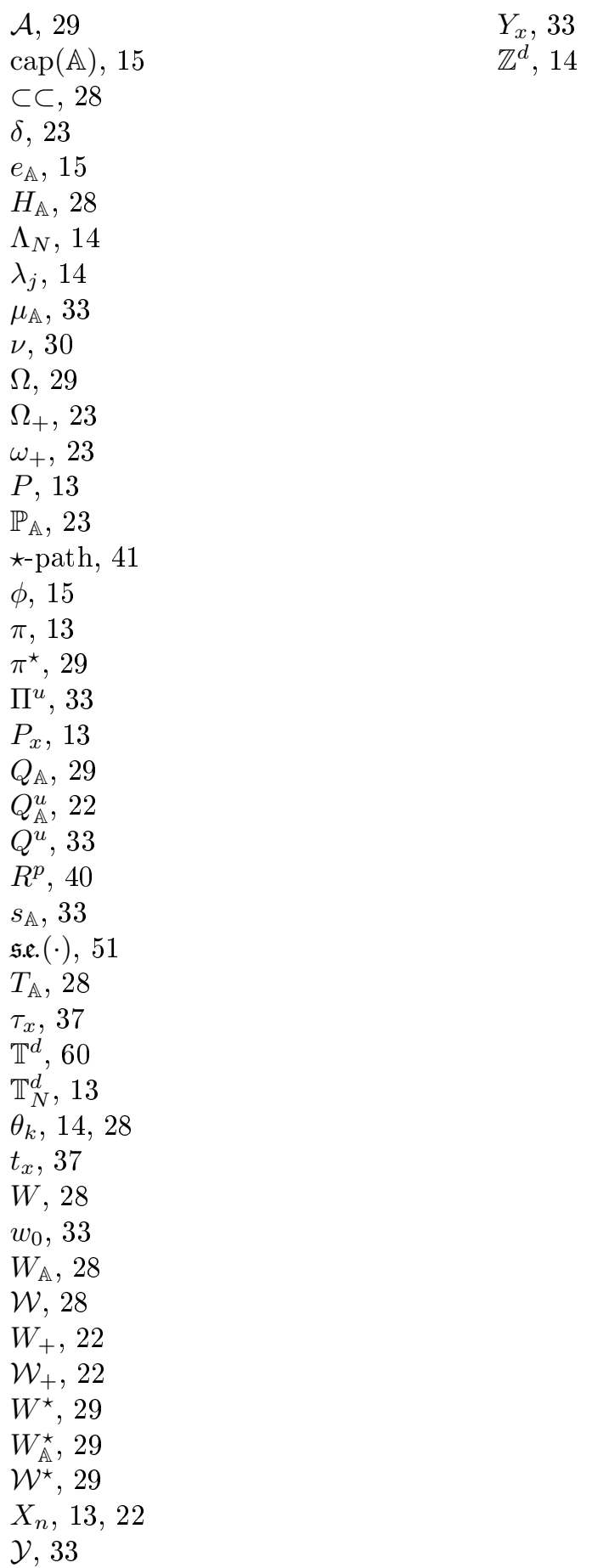




\section{Bibliography}

[1] David J. Aldous and Mark Brown. Inequalities for rare events in timereversible Markov chains. I. In Stochastic inequalities (Seattle, WA, 1991), volume 22 of IMS Lecture Notes Monogr. Ser., pages 1-16. Inst. Math. Statist., Hayward, CA, 1992.

[2] David J. Aldous and Mark Brown. Inequalities for rare events in timereversible Markov chains. II. Stochastic Process. Appl., 44(1):15-25, 1993.

[3] David Belius. Cover levels and random interlacements. Ann. Appl. Probab., 22(2):522-540, 2012.

[4] David Belius. Gumbel fluctuations for cover times in the discrete torus. arXiv:1202.0190, 2012.

[5] Itai Benjamini, Harry Kesten, Yuval Peres, and Oded Schramm. Geometry of the uniform spanning forest: transitions in dimensions 4, 8,12,... Ann. of Math. (2), 160(2):465-491, 2004.

[6] Itai Benjamini and Alain-Sol Sznitman. Giant component and vacant set for random walk on a discrete torus. J. Eur. Math. Soc. (JEMS), 10(1):133-172, 2008.

[7] Béla Bollobás. Random graphs, volume 73 of Cambridge Studies in Advanced Mathematics. Cambridge University Press, Cambridge, second edition, 2001.

[8] Béla Bollobás and Oliver Riordan. Percolation. Cambridge University Press, New York, 2006.

[9] Christian Borgs, Jennifer T. Chayes, Remco van der Hofstad, Gordon Slade, and Joel Spencer. Random subgraphs of finite graphs. I. The scaling window under the triangle condition. Random Structures Algorithms, 27(2):137-184, 2005. 
[10] Christian Borgs, Jennifer T. Chayes, Remco van der Hofstad, Gordon Slade, and Joel Spencer. Random subgraphs of finite graphs. II. The lace expansion and the triangle condition. Ann. Probab., 33(5):18861944, 2005.

[11] Robert Burton and Michael Keane. Density and uniqueness in percolation. Comm. Math. Phys., 121(3):501-505, 1989.

[12] Jiří Černý and Serguei Popov. On the internal distance in the interlacement set. Electron. J. Probab., 17(29):1-25, 2012.

[13] Jiří Černý and Augusto Teixeira. Critical window for the vacant set left by random walk on random regular graphs. to appear in Random Structures \& Algorithms, arXiv:1101.1978, 2011.

[14] Jiří Černý, Augusto Teixeira, and David Windisch. Giant vacant component left by a random walk in a random $d$-regular graph. Ann. Inst. H. Poincaré Probab. Statist., 47(4):929-968, 2011.

[15] Colin Cooper and Alan Frieze. Component structure induced by a random walk on a random graph. to appear in Random Structures \& Algorithms, arXiv 1005.1564, 2010.

[16] Amir Dembo and Alain-Sol Sznitman. On the disconnection of a discrete cylinder by a random walk. Probab. Theory Related Fields, 136(2):321-340, 2006.

[17] Amir Dembo and Alain-Sol Sznitman. A lower bound on the disconnection time of a discrete cylinder. In In and out of equilibrium. 2, volume 60 of Progr. Probab., pages 211-227. Birkhäuser, Basel, 2008.

[18] Richard Durrett. Probability: Theory and Examples. Duxbury Press, Belmont, CA, second edition, 1996.

[19] Paul Erdôs and Alfréd Rényi. On the evolution of random graphs. Magyar Tud. Akad. Mat. Kutató Int. Közl., 5:17-61, 1960.

[20] Geoffrey Grimmett. Percolation, volume 321 of Grundlehren der Mathematischen Wissenschaften. Springer-Verlag, Berlin, second edition, 1999.

[21] Olle Häggström and Johan Jonasson. Uniqueness and non-uniqueness in percolation theory. Probab. Surv., 3:289-344 (electronic), 2006.

[22] Hamed Hatami and Michael Molloy. The scaling window for a random graph with a given degree sequence. In Proceedings of SODA 2010, 2010. more exhaustive version of the paper appears at arXiv:0907.4211. 
[23] Marcelo Hilário, Vladas Sidoravicius, and Augusto Teixeira. Cylinders' percolation in three dimensions. arXiv:1202.1684, 2012.

[24] Svante Janson and Malwina J. Luczak. A new approach to the giant component problem. Random Structures Algorithms, 34(2):197-216, 2009 .

[25] Olav Kallenberg. Foundations of modern probability. Probability and its Applications (New York). Springer-Verlag, New York, second edition, 2002.

[26] Gregory F. Lawler. A self-avoiding random walk. Duke Math. J., 47(3):655-693, 1980.

[27] Gregory F. Lawler. Intersections of random walks. Probability and its Applications. Birkhäuser Boston Inc., Boston, MA, 1991.

[28] Gregory F. Lawler and Vlada Limic. Random walk: a modern introduction, volume 123 of Cambridge Studies in Advanced Mathematics. Cambridge University Press, Cambridge, 2010.

[29] David A. Levin, Yuval Peres, and Elizabeth L. Wilmer. Markov chains and mixing times. American Mathematical Society, Providence, RI, 2009. With a chapter by James G. Propp and David B. Wilson.

[30] Eyal Lubetzky and Allan Sly. Cutoff phenomena for random walks on random regular graphs. Duke Math. J., 153(3):475-510, 2010.

[31] Colin McDiarmid. Concentration. In Probabilistic methods for algorithmic discrete mathematics, volume 16 of Algorithms Combin., pages 195-248. Springer, Berlin, 1998.

[32] Jason Miller. Painting a graph with competing random walks. arXiv:1003.2168, 2010.

[33] Michael Molloy and Bruce Reed. A critical point for random graphs with a given degree sequence. In Proceedings of the Sixth International Seminar on Random Graphs and Probabilistic Methods in Combinatorics and Computer Science, "Random Graphs '93" (Poznań, 1993), volume 6, pages 161-179, 1995.

[34] Eviatar Procaccia and Eric Shellef. On the range of a random walk in a torus and random interlacements. arXiv:1007.1401, 2010.

[35] Eviatar B. Procaccia and Johan Tykesson. Geometry of the random interlacement. Electron. Commun. Probab., 16:528-544, 2011. 
[36] Balázs Ráth and Artëm Sapozhnikov. The effect of small quenched noise on connectivity properties of random interlacements. arXiv:1109.5086, 2011.

[37] Bálazs Ráth and Artëm Sapozhnikov. On the transience of random interlacements. Electronic Communications in Probability, 16:379391, 2011.

[38] Balázs Ráth and Artëm Sapozhnikov. Connectivity properties of random interlacement and intersection of random walks. ALEA Latin American Journal of Probability and Mathematical Statistics, 9:6783, 2012.

[39] Sidney I. Resnick. Extreme values, regular variation and point processes. Springer Series in Operations Research and Financial Engineering. Springer, New York, 2008. Reprint of the 1987 original.

[40] Vladas Sidoravicius and Alain-Sol Sznitman. Percolation for the vacant set of random interlacements. Comm. Pure Appl. Math., 62(6):831-858, 2009.

[41] Vladas Sidoravicius and Alain-Sol Sznitman. Connectivity bounds for the vacant set of random interlacements. Ann. Inst. Henri Poincaré Probab. Stat., 46(4):976-990, 2010.

[42] Frank Spitzer. Principles of random walks. Springer-Verlag, New York, second edition, 1976. Graduate Texts in Mathematics, Vol. 34.

[43] Alain-Sol Sznitman. How universal are asymptotics of disconnection times in discrete cylinders? Ann. Probab., 36(1):1-53, 2008.

[44] Alain-Sol Sznitman. On the domination of random walk on a discrete cylinder by random interlacements. Electron. J. Probab., 14:no. 56, 1670-1704, 2009.

[45] Alain-Sol Sznitman. Random walks on discrete cylinders and random interlacements. Probab. Theory Related Fields, 145(1-2):143-174, 2009.

[46] Alain-Sol Sznitman. Upper bound on the disconnection time of discrete cylinders and random interlacements. Ann. Probab., 37(5):17151746, 2009.

[47] Alain-Sol Sznitman. Vacant set of random interlacements and percolation. Ann. of Math. (2), 171(3):2039-2087, 2010.

[48] Alain-Sol Sznitman. Decoupling inequalities and interlacement percolation on $\mathcal{G} \times \mathbb{Z}$. Inventiones Mathematicae, 187:645-706, 2012. 10.1007/s00222-011-0340-9. 
[49] Martin Tassy. Random interlacements on Galton-Watson trees. Electron. Commun. Probab., 15:562-571, 2010.

[50] Augusto Teixeira. Interlacement percolation on transient weighted graphs. Electron. J. Probab., 14:no. 54, 1604-1628, 2009.

[51] Augusto Teixeira. On the size of a finite vacant cluster of random interlacements with small intensity. Probab. Theory Related Fields, 150(3-4):529-574, 2011.

[52] Augusto Teixeira and Johan Tykesson. Random interlacements and amenability. to appear in The Annals of Applied Probability, 2011.

[53] Augusto Teixeira and David Windisch. On the fragmentation of a torus by random walk. Comm. Pure Appl. Math., 64(12):1599-1646, 2011.

[54] Johan Tykesson and David Windisch. Percolation in the vacant set of poisson cylinders. to appear in Probability Theory and Related Fields, 2010.

[55] David Windisch. Logarithmic components of the vacant set for random walk on a discrete torus. Electron. J. Probab., 13:no. 28, 880-897, 2008.

[56] David Windisch. Random walk on a discrete torus and random interlacements. Electron. Commun. Probab., 13:140-150, 2008.

[57] David Windisch. Random walks on discrete cylinders with large bases and random interlacements. Ann. Probab., 38(2):841-895, 2010.

[58] Wolfgang Woess. Random walks on infinite graphs and groups, volume 138 of Cambridge Tracts in Mathematics. Cambridge University Press, Cambridge, 2000.

[59] Nick C. Wormald. Models of random regular graphs. In Surveys in combinatorics, 1999 (Canterbury), volume 267 of London Math. Soc. Lecture Note Ser., pages 239-298. Cambridge Univ. Press, Cambridge, 1999. 
Jiří Černý

Faculty of Mathematics, University of Vienna

Nordbergstrasse 15 ,

1090 Vienna, Austria

jiri.cerny@univie.ac.at

http://www.mat.univie.ac.at/〜cerny

Augusto Quadros Teixeira

Instituto Nacional de Matemática Pura e Aplicada (IMPA)

Estrada Dona Castorina, 110,

22460-320 - Rio de Janeiro, Brazil

augusto@impa.br

http://www.impa.br/ augusto 